Rev. Mat. Iberoamericana 22 (2006), no. 3, 893-953

\title{
Recouvrements, derivation des mesures et dimensions
}

\section{Patrice Assouad et Thierry Quentin de Gromard}

\begin{abstract}
Let $X$ be a set with a symmetric kernel $d$ (not necessarily a distance). The space $(X, d)$ is said to have the weak (resp. strong) covering property of degree $\leq m$ [briefly $\operatorname{prf}(m)$ (resp. $\operatorname{prF}(m))$ ], if, for each family $\mathcal{B}$ of closed balls of $(X, d)$ with radii in a decreasing sequence (resp. with bounded radii), there is a subfamily, covering the center of each element of $\mathcal{B}$, and of order $\leq m$ (resp. spliting into $m$ disjoint families). Since Besicovitch, covering properties are known to be the main tool for proving derivation theorems for any pair of measures on $(X, d)$.

Assuming that any ball for $d$ belongs to the Baire $\sigma$-algebra for $d$, we show that the prf implies an almost sure derivation theorem. This implication was stated by D. Preiss when $(X, d)$ is a complete separable metric space. With stronger measurability hypothesis (to be stated later in this paper), we show that the prf restricted to balls with constant radius implies a derivation theorem with convergence in measure.

We show easily that an equivalent to the $\operatorname{prf}(m+1)$ (resp. to the $\operatorname{prf}(m+1)$ restricted to balls with constant radius) is that the Nagatadimension (resp. the De Groot-dimension) of $(X, d)$ is $\leq m$. These two dimensions (see J.I. Nagata) are not lesser than the topological dimension ; for $\mathbb{R}^{n}$ with any given norm $(n>1)$, they are $>n$. For spaces with nonnegative curvature $\geq 0$ (for example for $\mathbb{R}^{n}$ with any given norm), we express these dimensions as the cardinality of a net ; in these spaces, we give a similar upper bound for the degree of the prF (generalizing a result of Furedi and Loeb for $\mathbb{R}^{n}$ ) and try to obtain the exact degree in $\mathbb{R}$ and $\mathbb{R}^{2}$.
\end{abstract}

2000 Mathematics Subject Classification: 28A15.

Keywords: covering properties, derivation theorems, dimensions of metric spaces. 


\section{Introduction}

Soit $X$ un ensemble muni d'un noyau symétrique $d$; nous dirons que $(X, d)$ possède la propriété de recouvrement faible (resp. forte) de degré $\leq m$ si de toute famille $\mathcal{B}$ de boules fermées de $(X, d)$ à rayons dans une suite décroissante (resp. à rayons bornés), on peut extraire une sous-famille recouvrant les centres de $\mathcal{B}$ et d'ordre $\leq m$ (resp. et décomposable en $m$ familles disjointes). Les autres notions mentionnées dans cette introduction seront définies plus tard (voir la table à la fin de cet article).

Les propriétés de recouvrement sont l'outil essentiel de démonstration des théorèmes de dérivation pour des mesures quelconques sur un espace métrique $(X, d)$, et ceci depuis le travail initial de Besicovitch [6] concernant $\mathbb{R}^{2}$ :

- la propriété de recouvrement forte est la plus classiquement utilisée ([6], [28], [20], [18], [19], [42]) ; on en déduit le théorème de recouvrement de Vitali (recouvrement presque sûr par des boules disjointes), puis une inégalité maximale, puis le théorème de dérivation ;

- une autre propriété de recouvrement (propriété de De Giorgi) est utilisée dans [16] et [41] pour établir d'une autre manière le théorème de dérivation.

Une voie plus directe consiste à utiliser la propriété de recouvrement faible (voir De Guzman [19, pp. 40-43], et surtout Preiss [33], dans le cas des espaces métriques séparables complets).

Les objectifs de cet article sont multiples. Nous considérons un noyau symétrique $d$ sur $X$, qui n'est pas nécessairement une distance et nous obtenons alors les résultats suivants.

\section{A. Théorème de dérivation presque sûre :}

On fait sur le noyau $d$ des hypothèses de mesurabilité minimales : on suppose seulement que les boules pour $d$ sont dans la tribu de Baire de $d$. Nous montrons qu'alors la propriété de recouvrement faible implique un théorème de dérivation presque sûre.

En particulier, notre démonstration traite complètement le cas où $(X, d)$ est un espace métrique séparable complet possédant la propriété de recouvrement faible ; c'est le cas envisagé dans une très intéressante note de David Preiss [33], essentiellement consacrée à démontrer une réciproque ; mais il n'y donne pas la démonstration du théorème de dérivation, renvoyant à des arguments "classiques" qui, nous le pensons après avoir écrit la démonstration, ne sont pas tellement "classiques". 
Loin d'être une conséquence des théorèmes de convergence de martingales, ce théorème de dérivation presque sûre implique la convergence des martingales équi-intégrables discrètes relatives à une filtration discrète (il s'agit du cas où $d$ est une ultramétrique).

B. Théorème de dérivation en mesure :

On fait sur le noyau $d$ des hypothèses de mesurabilité beaucoup plus fortes qu'en A (qui seront précisées en temps opportun) : elles sont satisfaites notamment lorsque le noyau $d$ est bi-Lipschitz équivalent à une distance $\delta$ telle que $(X, \delta)$ soit séparable, et que les boules pour $d$ sont dans la tribu de Baire de $\delta$.

Nous montrons alors que la propriété de recouvrement faible restreinte aux boules à rayon constant implique un théorème de dérivation en mesure. Ce type de résultat est nouveau, et sa démonstration est un peu inspirée d'une démonstration de Mattila [26] dans $\mathbb{R}^{n}$.

C. Liens entre propriétés de recouvrement et dimensions :

Nous montrons aisément que la propriété de recouvrement faible de degré $\leq m+1$ équivaut à ce que la dimension de Nagata soit $\leq m$; de même la propriété de recouvrement faible de degré $\leq m+1$ restreinte aux boules à rayon constant équivaut à ce que la dimension de De Groot soit $\leq m$. Ces deux dimensions, définies plus loin, sont supérieures ou égales à la dimension topologique (voir Nagata [30]) ; pour $\mathbb{R}^{n}$ muni d'une norme quelconque $(n>1)$, elles sont $>n$.

D. Calcul du degré des propriétés de recouvrement:

Pour les espaces à courbure $\geq 0$, et notamment pour $\mathbb{R}^{n}$ muni d'une norme quelconque, nous calculons ces deux dimensions en termes de cardinalité de certains réseaux, et nous montrons qu'elles sont égales. Nous majorons aussi dans ce cas le degré de la propriété de recouvrement forte par le cardinal d'un réseau, étendant un résultat de Furedi et Loeb [21] valable pour $\mathbb{R}^{n}$. On doit avoir à l'esprit que la notion d'espace " $\grave{a}$ courbure $\geq 0$ " que nous utilisons est beaucoup plus vague que la notion usuelle (voir 1.5).

\section{E. Equivalence entre propriétés de recouvrement:}

Nous montrons que les propriétés de recouvrement faible et forte sont équivalentes dès que le noyau $d$ possède la "doubling property" et que les boules pour le noyau $d$ sont suffisamment régulières. 
F. Quelques applications :

1. La dimension de De Groot est finie dès que le noyau $d$ possède la "doubling property" ; dans ce cas, sous les hypothèses de mesurabilité mentionnées en $\mathbf{B}$, on a un théorème de dérivation en mesure ; cela s'applique notamment au groupe de Heisenberg muni de sa distance usuelle, dont la dimension de Nagata est infinie. Notons que l'équivalence bi-lipschitzienne, qui conserve la "doubling property", ne conserve pas la finitude de la dimension de Nagata.

2. Si le noyau $d$ est la borne inférieure de $n+1$ ultramétriques, sa dimension de Nagata est $\leq n$; en général $d$ n'est pas une distance, et ne vérifie même pas les hypothèses de mesurabilité mentionnées en $\mathbf{A}$; cependant si $d$ vérifie ces hypothèses (par exemple si le noyau $d$ est bi-Lipschitz équivalent à une distance $\delta$, et que les boules pour $d$ sont dans la tribu de Baire de $\delta$ ), alors on a un théorème de dérivation presque sûre.

3. En particulier on peut construire sur $\mathbb{R}^{n}$ un noyau $d$ bi-Lipschitz équivalent à la distance euclidienne, borne inférieure de $n+1$ ultramétriques et dont les boules ouvertes sont des ouverts de $\mathbb{R}^{n}$ (nous décrirons cette construction dans un article ultérieur).

4. Soit $\lambda$ une loi de probabilité sur un ensemble $X$ muni d'un noyau $d$ et d'une $\sigma$-algèbre contenant les réunions quelconques de boules ouvertes de $d$. Nous montrons que si $(X, d)$ a une partie dense de cardinal petit au sens d'Ulam et possède pour chaque $r$ la propriété de recouvrement faible restreinte aux boules de rayon $r$, alors l'ensemble des centres des boules ouvertes $\lambda$-négligeables est lui-même $\lambda$-négligeable.

Une bonne partie des résultats présentés subsiste si on remplace les boules fermées par des boules ouvertes (voir 2.6.10), ou même par des couples $(x, B)$ (qu'on peut appeler des pseudoboules) d'une partie mesurable bornée $B$ et d'un point $x \in B$; pour les pseudoboules cela sera l'objet d'un travail ultérieur. Le plan de ce travail est le suivant :

1. Introduction (dans laquelle on se trouve).

2. Préliminaires.

3. Dérivation des mesures (convergence en mesure).

4. Dérivation des mesures (convergence presque sûre).

5. Propriété de recouvrement forte dans les espaces à courbure $\geq 0$.

6. Dimension métrique et propriétés de recouvrement.

7. Tableau des principales notions et notations. 


\section{Notations}

On note $\mathbf{I}_{A}$ l'indicatrice de $A$ et $a \vee b$ (resp. $a \wedge b$ ) le maximum (resp. le minimum) de $a$ et $b$.

\section{(1.1) Familles de boules}

Soit $X$ un ensemble muni d'un noyau symétrique $d$ (voir 1.3), par exemple un espace métrique $(X, d)$. Pour chaque $y \in X$ et $r \in] 0,+\infty[$, on pose

$$
\begin{aligned}
& \mathbf{B}_{d}(y, r)=\{x \in X \mid d(x, y) \leq r\} \quad \text { (boule fermée), } \\
& \mathbf{U}_{d}(y, r)=\{x \in X \mid d(x, y)<r\} \quad \text { (boule ouverte) } \\
& \text { et } \mathbf{S}_{d}(y, r)=\{x \in X \mid d(x, y)=r\} \quad \text { (sphère) }
\end{aligned}
$$

(précisons bien que le rayon d'une boule ou d'une sphère est toujours fini et $>0)$.

(a) Une famille $\mathcal{B}=\left(\mathbf{B}_{d}\left(y_{i}, r_{i}\right)\right)_{i \in I}$ est dite à rayons bornés (resp. à rayons discrets) si les $\left(r_{i}\right)_{i \in I}$ sont bornés (resp. si les $\left(r_{i}\right)_{i \in I}$ sont dans une suite décroissante $\left.\left(v_{k}\right)_{k \geq 1}\right)$. Si $r_{i}$ est constant, on dit que $\mathcal{B}$ est $\grave{a}$ rayon constant.

(b) Une famille $\mathcal{B}$ de parties de $(X, d)$ est dite d'ordre $\leq m$ si tout $a \in X$ est contenu dans au plus $m$ éléments de $\mathcal{B}$; on note $o(\mathcal{B})$ la valeur optimale de $m$.

(c) Une famille $\mathcal{B}$ de parties de $(X, d)$ est dite colorable en $m$ couleurs si elle est réunion de $m$ familles de boules disjointes ; on note $\chi(\mathcal{B})$ le nombre minimum de couleurs nécessaire.

\section{(1.2) Propriétés de recouvrement}

Soit $X$ un ensemble muni d'un noyau symétrique $d$ (voir 1.3), par exemple un espace métrique $(X, d)$.

(a) On dit que $(X, d)$ possède la propriété de recouvrement faible de degré $\leq m$ (resp. la propriété de De Giorgi de degré $\leq m$ ) s'il vérifie :

de toute famille $\mathcal{B}$ de boules fermées de $(X, d)$ à rayons discrets (resp. bornés), on peut extraire une sous-famille recouvrant les centres de $\mathcal{B}$ et d'ordre $\leq m$.

La valeur optimale de $m$ est appelée $\mathbf{n}_{D}$ (resp. $\mathbf{n}_{B}$ ).

On abrège "propriété de recouvrement faible de degré $\leq m$ " en $\operatorname{prf}(m)$. 
(b) Soit $\mathcal{P}$ une propriété concernant les familles de boules fermées ; on dit que $(X, d)$ possède la propriété de recouvrement faible de degré $\leq m$ restreinte aux familles de boules fermées vérifiant $\mathcal{P}$ (en bref $\operatorname{prf}(m)$ pour les boules vérifiant $\mathcal{P}$ ) s'il vérifie:

de toute famille $\mathcal{B}$ de boules fermées de $(X, d)$ à rayons discrets et telle que $\mathcal{B}$ vérifie $\mathcal{P}$, on peut extraire une sous-famille $\mathcal{E}$ recouvrant les centres de $\mathcal{B}$ et d'ordre $\leq m$.

Indiquons deux cas particuliers qui seront fréquemment utilisés :

(b1) si $(X, d)$ possède la propriété de recouvrement faible de degré $\leq m$ restreinte aux familles de boules fermées à rayon constant (en bref $\operatorname{prf}(m)$ pour les boules à rayon constant), la valeur optimale de $m$ est appelée $\mathbf{n}_{C}$;

(b2) soit $Y \subset X$; quand on dit que $(X, d)$ possède la propriété de recouvrement faible de degré $\leq m$ restreinte aux familles de boules fermées centrées dans $Y$ (en bref $\operatorname{prf}(m)$ pour les boules centrées dans $Y$ ), il est bien entendu que l'ordre de la sous-famille $\mathcal{E}$ est calculé dans $X$ et pas seulement dans $Y$, i.e. que cet ordre est $\leq m$ si et seulement si tout $a \in X$ est contenu dans au plus $m$ éléments de $\mathcal{E}$.

(c) On dit que $(X, d)$ possède la propriété de recouvrement forte de degré $\leq m$ s'il vérifie :

de toute famille $\mathcal{B}$ de boules fermées de $(X, d)$ à rayons bornés, on peut extraire une sous-famille recouvrant les centres de $\mathcal{B}$ et colorable en $m$ couleurs.

La valeur optimale de $m$ est appelée $\mathbf{N}_{B}$, ou $\mathbf{N}_{D}$ (resp. $\mathbf{N}_{C}$ ) si on se restreint aux familles de boules à rayons discrets (resp. à rayon constant).

(d) On a évidemment $\mathbf{n}_{C} \leq \mathbf{n}_{D} \leq \mathbf{n}_{B}$ et $\mathbf{N}_{C} \leq \mathbf{N}_{D} \leq \mathbf{N}_{B}$; on a aussi $\mathbf{n}_{C} \leq \mathbf{N}_{C}, \mathbf{n}_{D} \leq \mathbf{N}_{D}$ et $\mathbf{n}_{B} \leq \mathbf{N}_{B}$

\section{(1.3) Noyaux et $\sigma$-algèbres}

(a) Un noyau symétrique $d$ sur un ensemble $X$ est une application de $X^{2}$ dans $[0,+\infty]$ vérifiant $d(x, y)=d(y, x)$ et $d(x, x)=0$ pour tous $x, y \in X$; c'est un écart si on a de plus $d(x, y) \leq d(x, z)+d(z, y)$ pour tous $x, y, z \in X$.

(b) Si le noyau $d$ n'est pas une distance ou un écart, on doit préciser que les ouverts de la topologie $\mathcal{T}_{d}$ associée à $d$ sont les ensembles $V$ de la 
forme $\cup_{y \in V} \mathbf{B}_{d}\left(y, r_{y}\right)$, avec $\left.r_{y} \in\right] 0,+\infty[$ pour tout $y \in V$. En général les boules $\mathbf{B}_{d}(x, r)$ (resp. $\left.\mathbf{U}_{d}(x, r)\right)$ ne sont pas fermées (resp. ouvertes) dans $\left(X, \mathcal{T}_{d}\right)$.

(c) On note $\mathcal{A}_{d}$ (resp. $\mathcal{B}_{d}$ ) la tribu, ou $\sigma$-algèbre, engendrée par les boules pour $d$ (resp. par les fonctions numériques continues bornées $\left.\operatorname{sur}\left(X, \mathcal{T}_{d}\right)\right)$. La tribu $\mathcal{B}_{d}$ est appelée tribu de Baire et ne dépend que de $\mathcal{T}_{d}$

De plus, quel que soit $s>0$, les boules fermées de rayon $\leq s$ pour $d$ engendrent $\mathcal{A}_{d}$.

(d) Si $d$ est un écart, on a $\mathcal{A}_{d} \subset \mathcal{B}_{d}$, et $\mathcal{B}_{d}$ est égale à la tribu borélienne (engendrée par les ouverts). Si $(X, d)$ est un espace métrique séparable, on a $\mathcal{A}_{d}=\mathcal{B}_{d}$.

(e) Deux noyaux symétriques $d$ et $\delta$ sur $X$ sont dits bi-Lipschitz équivalents s'il existe $A, B \in] 0,+\infty[$ avec $A \delta \leq d \leq B \delta$.

Des espaces métriques plus particuliers seront utilisés aux paragraphes 5 et 6 et, à titre d'exemple, au paragraphe 4 :

\section{(1.4) Espaces géodésiques et courbure}

(a) Un espace métrique $(X, d)$ est dit géodésique si tout couple de points peut être joint par au moins un segment géodésique (i.e. une image isométrique dans $(X, d)$ d'un segment de $\mathbb{R})$.

(b) Un espace métrique $(X, d)$ est dit à courbure $\geq 0$ s'il est géodésique et si, pour tous $a, x, y \in X$ et tous $[a, x],[a, y]$ des segments géodésiques joignant $a$ respectivement à $x$ et à $y$, on a $d\left(x_{t}, y_{t}\right) \geq t d(x, y)$ pour tout $t$ assez petit, où $x_{t}$ (resp. $\left.y_{t}\right)$ désigne le point de $[a, x]$ (resp. $[a, y]$ ) situé à la distance $t d(a, x)$ (resp. $t d(a, y))$ de $a$.

(c) Exemples d'espaces à courbure $\geq 0$ :

- tout espace normé,

- toute variété riemannienne complète connexe à courbure sectionnelle $\geq 0$ (théorème de Toponogov, [35] [10] [7]).

Ce que nous appelons "espace à courbure $\geq 0$ " est quelque chose de beaucoup plus vague (plus général) que la notion usuelle ; en effet, les espaces normés de norme quelconque sont des espaces à courbure $\geq 0$ en notre sens, mais pas au sens usuel (voir par exemple [9], p 103). Il eut été peut-être plus prudent de notre part d'employer une autre terminologie. 


\section{Préliminaires}

On va donner ici certains préliminaires indispensables dans les paragraphes suivants. Les résultats principaux se trouvent en 2.3 et 2.4 (approximation par des fonctions continues), en 2.5 (parties chargées par une mesure), en 2.6 (mesures portées par leur support) et en 2.7 (mesurabilité).

\section{(2.1) Mesures et quotients de dérivation}

Soit $X$ un ensemble muni d'un noyau symétrique $d$; soit $\mathcal{A}$ une tribu sur $X$ avec $\mathcal{A} \supset \mathcal{A}_{d}$.

(a) On note $\mathcal{M}_{1}(X, \mathcal{A})$ (resp. $\mathcal{M}_{1}^{+}(X, \mathcal{A})$ ) l'ensemble des mesures finies (resp. des mesures $\geq 0$ finies) $\operatorname{sur}(X, \mathcal{A})$. Pour $\lambda \in \mathcal{M}_{1}^{+}(X, \mathcal{A})$ et $f \in L^{1}(X, \mathcal{A}, \lambda)$, on note $f \lambda$ la mesure $A \mapsto \int_{A} f(y) \lambda(d y)$.

On note $\mathcal{P}(X, \mathcal{A})$ l'ensemble des $\lambda \in \mathcal{M}_{1}^{+}(X, \mathcal{A})$ avec $\lambda(X)=1$.

(b) (quotients de dérivation)

Soient $\lambda \in \mathcal{M}_{1}^{+}(X, \mathcal{A}), \nu \in \mathcal{M}_{1}(X, \mathcal{A})$ et $\left.r \in\right] 0,+\infty[;$ on pose alors :

$$
\begin{aligned}
T_{r}(\nu, \lambda)(y) & =\nu\left(\mathbf{B}_{d}(y, r)\right) / \lambda\left(\mathbf{B}_{d}(y, r)\right) \\
\text { et } \quad S_{r}(\nu, \lambda)(y) & =\sup _{0<u<r} T_{u}(\nu, \lambda)(y) .
\end{aligned}
$$

On pose $T_{r}(\nu, \lambda)(y)=0$ si $\nu\left(\mathbf{B}_{d}(y, r)\right)=\lambda\left(\mathbf{B}_{d}(y, r)\right)=0$.

(c) On note $Z(\lambda, d)$ l'ensemble des $y \in X$ tels qu'il existe $r>0$ avec $\lambda\left(\mathbf{B}_{d}(y, r)\right)=0 ; Z(\lambda, d)$ est ouvert dans $\left(X, \mathcal{T}_{d}\right)$ dès que chaque boule de $(X, d)$ est un voisinage de son centre dans $\left(X, \mathcal{T}_{d}\right)$. On étudiera en 2.6 dans quels cas $Z(\lambda, d)$ est $\lambda$-négligeable.

(d) Soient $\lambda \in \mathcal{P}(X, \mathcal{A})$ et $f \lambda$-intégrable ou positive ; on pose $\mathbb{E}_{\lambda}(f)=$ $\int f(y) \lambda(d y) ;$ si $\mathcal{C}$ est une sous-tribu de $\mathcal{A}$, on note $\mathbb{E}_{\lambda}(f \mid \mathcal{C})$ une version de l'espérance conditionnelle de $f$ par rapport à $\mathcal{C}$ (et à la loi $\lambda$ ). On renvoie aux deux premiers chapitres de Meyer [27] et à Dellacherie-Meyer ([14] et [15]) pour tout ce qui concerne la théorie de la mesure.

\section{(2.2) Intégrales supérieures}

Soit $X$ un ensemble muni d'un noyau symétrique $d$; soit $\mathcal{A}$ une tribu sur $X$ avec $\mathcal{A} \supset \mathcal{A}_{d}$.

Soient $\lambda \in \mathcal{M}_{1}^{+}(X, \mathcal{A})$ et $f \geq 0$ non nécessairement $\mathcal{A}$-mesurable ; on note $\int^{*} f(y) \lambda(d y)$ ou $\mathbb{E}_{\lambda}^{*}(f)$ son intégrale supérieure. On pose $\lambda^{*}(A)=$ $\mathbb{E}_{\lambda}^{*}\left(\mathbf{I}_{A}\right)$ et $\int_{A}^{*} f(y) \lambda(d y)=\mathbb{E}_{\lambda}^{*}\left(f \mathbf{I}_{A}\right)$. 
Pour tout ce qui touche à l'intégrale supérieure, on renvoie à la première partie du livre de Van der Vaart et Wellner [40]. Cependant on en rappelle la définition et les principales propriétés :

- pour $f \geq 0, \int^{*} f(y) \lambda(d y)$ est la borne inférieure de $\int g(y) \lambda(d y)$ pour $g$ mesurable $\geq f$; cette borne est atteinte pour une fonction $g$ unique à une fonction $\lambda$-négligeable près, qu'on notera $f^{*}$;

- l'application $f \mapsto \int^{*} f(y) \lambda(d y)$ est croissante et sous-additive ;

- pour $0 \leq f_{n} \nearrow f$, on a $\int^{*} f_{n}(y) \lambda(d y) \nearrow \int^{*} f(y) \lambda(d y)$.

On précisera en 3.2 quelques difficultés concernant la convergence en mesure dans ce cadre.

\section{(2.3) Approximation des fonctions mesurables}

Le résultat d'approximation 2.3.2 qui suit est sûrement classique (voir par exemple [14, pp.19-24]). Comme ses corollaires 2.4a et 2.4c pour la tribu de Baire jouent un rôle important pour démontrer les théorèmes de dérivation, on a préféré en donner une démonstration.

(2.3.1) Soit $X$ un ensemble et soit $E$ un espace vectoriel de fonctions bornées de $X$ dans $\mathbb{R}$. On note $E^{\wedge}$ l'ensemble des fonctions de $X$ dans $\left[-\infty,+\infty\left[\right.\right.$ qui sont borne inférieure d'une suite d'éléments de $E$. On note $\mathcal{B}_{E}$ la tribu engendrée par $E$.

Proposition 2.3.2. Soit $X$ un ensemble et soit $E$ un espace vectoriel de fonctions bornées de $X$ dans $\mathbb{R}$ contenant les constantes, stable par $\vee$ fini, par $\wedge$ fini et par limite uniforme. Soit $\mathcal{A}$ une tribu sur $X$ contenant $\mathcal{B}_{E}$ et soit $\lambda \in \mathcal{P}(X, \mathcal{A})$. On a alors les propriétés suivantes :

(a) pour tout $B \in \mathcal{B}_{E}$ et tout $\varepsilon>0$, il existe $g \in E$ avec

$$
\{g \geq 0\} \subset B \quad \text { et } \quad \lambda(B)<\lambda(\{g \geq 0\})+\varepsilon ;
$$

(b) pour tout $f \mathcal{B}_{E}$-mesurable, $\lambda$-intégrable et partout finie, pour tout $\varepsilon>0$, il existe $g \in E^{\wedge}$ avec $g \leq f$ et $\mathbb{E}_{\lambda}(f-g)<\varepsilon ;$

(c) pour tout $f \mathcal{B}_{E}$-mesurable, $\lambda$-intégrable et partout finie, pour tout $\varepsilon>0$, il existe $h \in E$ avec $\mathbb{E}_{\lambda}(|f-h|)<\varepsilon ;$

Démonstration. (a) Soit $B \in \mathcal{B}_{E}$; alors, pour tout $\varepsilon>0$, il existe $g, h \in E$ avec

$$
\{g \geq 0\} \subset B \subset\{h>0\} \quad \text { et } \quad \lambda(\{h>0\})<\lambda(\{g \geq 0\})+\varepsilon .
$$

Pour le montrer, on remarque que l'ensemble $\mathcal{C}$ des $B$ vérifiant cette propriété forme une tribu contenant $\{f \geq 0\}$ pour tout $f \in E$ : en effet le complé- 
mentaire de tout élément de $\mathcal{C}$ est clairement dans $\mathcal{C}$; de plus toute réunion dénombrable d'éléments de $\mathcal{C}$ est dans $\mathcal{C}$, car $\bigcup_{n \in \mathbb{N}}\left\{h_{n}>0\right\}=\{h>0\}$ avec $h=\sum_{n \in \mathbb{N}} \varepsilon_{n} h_{n}$ pour des $\varepsilon_{n}>0$ convenables, et $\bigcup_{n \in \mathbb{N}}\left\{g_{n} \geq 0\right\}$ est approché par $\left\{\vee_{n \leq p} g_{n} \geq 0\right\}$ pour $p$ assez grand ; enfin, pour tout $f \in E$ et $a \in \mathbb{R}$, on a $\{f \geq a\}=\bigcap_{n \in \mathbb{N}}\{n(f-a)+1>0\}$ donc $\{f \geq a\} \in \mathcal{C}$.

(b) On suppose d'abord que $f$ est positive. Soit $\varepsilon>0$.

Soit $g_{0}=\sum_{k \in K} c_{k} \mathbf{I}_{B_{k}}$ une fonction étagée $\left(K\right.$ fini, $B_{k} \in \mathcal{B}_{E}$ et $c_{k} \geq 0$ ) avec $0 \leq g_{0} \leq f$ et $\mathbb{E}_{\lambda}\left(f-g_{0}\right)<\varepsilon$.

Utilisant (a), pour chaque $\alpha>0$ et chaque $k \in K$, on se donne $g_{k, \alpha} \in E$ avec $\left\{g_{k, \alpha} \geq 0\right\} \subset B_{k}$ et $\lambda\left(B_{k}\right)<\lambda\left(\left\{g_{k, \alpha} \geq 0\right\}\right)+\alpha$; si $\alpha$ est assez petit, la fonction $g=\sum_{k \in K} c_{k} \mathbf{I}_{\left\{g_{k, \alpha} \geq 0\right\}}$ répond à la question (car $\mathbf{I}_{\left\{g_{k, \alpha} \geq 0\right\}}=$ $\inf _{n \in \mathbb{N}}\left[\left(n g_{k, \alpha}+1\right)^{+} \wedge 1\right]$ appartient à $\left.E^{\wedge}\right)$.

Si $f$ est de signe quelconque, on choisit, pour chaque $n \in \mathbb{N}$, une fonction $g_{n} \in E^{\wedge} \lambda$-intégrable avec $0 \leq g_{n} \leq(f+n)^{+}$et $\mathbb{E}_{\lambda}\left((f+n)^{+}-g_{n}\right)<\varepsilon 2^{-n-1}$ pour tout $n \in \mathbb{N}$; alors la fonction $g=\inf _{n \in \mathbb{N}}\left(g_{n}-n\right)$ vérifie :

$$
g \in E^{\wedge}, \quad g \leq f \quad \text { et } \quad \mathbb{E}_{\lambda}(f-g)<\varepsilon .
$$

(c) On choisit $g \in E^{\wedge}$ comme dans (b) ; pour chaque $\alpha>0$, il existe $h \in E$ avec $g \leq h$ et $\mathbb{E}_{\lambda}(h-g)<\alpha$; si $\alpha$ est assez petit, la fonction $h$ répond à la question.

\section{(2.4) Approximation des fonctions Baire-mesurables}

(2.4.1) Soit $(X, \mathcal{T})$ un espace topologique ; on va appliquer le résultat précédent à l'espace vectoriel $E$ des fonctions continues bornées de $(X, \mathcal{T})$ dans $\mathbb{R}$. La tribu $\mathcal{B}_{E}$ est alors la tribu de Baire $\mathcal{B}(\mathcal{T})$ associée à $\mathcal{T}$.

Soit $\mathcal{A}$ une tribu sur $X$ contenant $\mathcal{B}(\mathcal{T})$. Soit $\lambda \in \mathcal{P}(X, \mathcal{A})$ et soit $f$ une fonction $\mathcal{B}(\mathcal{T})$-mesurable, $\lambda$-intégrable et partout finie. De 2.3.2, on tire les propriétés suivantes:

(a) pour tout $B \in \mathcal{B}(\mathcal{T})$ et tout $\varepsilon>0$, il existe $F \in \mathcal{B}(\mathcal{T})$ fermé dans $(X, \mathcal{T})$ et $G \in \mathcal{B}(\mathcal{T})$ ouvert dans $(X, \mathcal{T})$ avec $F \subset B \subset G$ et $\lambda(G)<\lambda(F)+\varepsilon$

(b) pour tout $f \mathcal{B}(\mathcal{T})$-mesurable, $\lambda$-intégrable et partout finie, et pour tout $\varepsilon>0$, il existe $g, h \mathcal{B}(\mathcal{T})$-mesurables vérifiant :

$g$ est s.c.s. majorée de $(X, \mathcal{T})$ dans $[-\infty,+\infty[$,

$h$ est s.c.i. minorée de $(X, \mathcal{T})$ dans $]-\infty,+\infty]$,

$g \leq f \leq h$ et $\mathbb{E}_{\lambda}(h-g)<\varepsilon ;$

l'énoncé ci-dessus est souvent appelé théorème de Vitali-Carathéodory; pour le cas où $\mathcal{T}$ est la topologie associée à une distance $d$, on le trouve dans $[14$, p. 23] ; 
(c) pour tout $f \mathcal{B}(\mathcal{T})$-mesurable, $\lambda$-intégrable et partout finie, et pour tout $\varepsilon>0$, il existe $h$ continue bornée sur $(X, \mathcal{T})$ avec $\mathbb{E}_{\lambda}(|f-h|)<\varepsilon$.

Si $\mathcal{T}$ est la topologie $\mathcal{T}_{d}$ associée à un noyau symétrique $d$, on peut tirer de l'encadrement 2.4.1b une approximation de $\mathbb{E}_{\lambda}(f)$ par des sommes de Riemann contrôlées par une jauge.

(2.4.2) On appelle jauge sur $X$ toute application $\gamma: x \in X \longmapsto \gamma(x)>0$; c'est une autre façon de voir le recouvrement de $X$ par les boules $\mathbf{B}_{d}(x, \gamma(x))$ pour $x \in X$.

On dit que $\mathcal{P}=\left(A_{i}, x_{i}\right)_{i \in I}$ est une $\mathcal{A}$-partition pointée de $X$ si $\left(A_{i}\right)_{i \in I}$ est une partition dénombrable et $\mathcal{A}$-mesurable de $X$ et si $\left(x_{i}\right)_{i \in I}$ est une famille de points de $X$ (on ne demande pas $x_{i} \in A_{i}$ ); on dit que $\mathcal{P}$ est $\gamma$-fine sur $(X, d)$ si on a $A_{i} \subset \mathbf{U}_{d}\left(x_{i}, \gamma\left(x_{i}\right)\right)$ pour tout $i \in I$.

La proposition suivante, intéressante en soi, est à mettre en rapport avec des résultats de [29], [13] et [34] ; elle pourrait conduire à une autre démonstration des théorèmes de dérivation (voir 3.7).

Proposition 2.4.3. Soit $X$ un ensemble muni d'un noyau symétrique $d$ avec $\mathcal{A}_{d} \subset \mathcal{B}_{d}$. Soit $\mathcal{A}$ une tribu sur $X$ avec $\mathcal{A} \supset \mathcal{B}_{d}$ et soit $\lambda \in \mathcal{P}(X, \mathcal{A})$. Soit $f$ une fonction $\mathcal{B}_{d}$-mesurable, partout finie et $\lambda$-intégrable de $X$ dans $\mathbb{R}$. On a alors :

(a) pour tout $\varepsilon>0$, il existe une jauge $\gamma$ et une mesure positive $\mu$ de masse totale $\leq 1$ telles que:

$$
|f \lambda(A)-f(y) \lambda(A)| \leq \varepsilon \mu(A) \text { dès que } A \subset \mathbf{U}_{d}(y, \gamma(y)), A \in \mathcal{B}_{d}
$$

(sans qu'on ait nécessairement $y \in A$ ).

(b) pour tout $\varepsilon>0$, il existe une jauge $\gamma$ sur $X$ telle que :

$$
\left|\mathbb{E}_{\lambda}(f)-\sum_{i \in I} f\left(x_{i}\right) \lambda\left(A_{i}\right)\right|<\varepsilon,
$$

pour toute $\mathcal{A}$-partition pointée $\gamma$-fine $\left(A_{i}, x_{i}\right)_{i \in I} \operatorname{sur}(X, d)$.

Démonstration. La démonstration de (a) résulte des trois lemmes 2.4.4, 2.4.5 et 2.4.6, qui suivent. De plus (b) est une conséquence immédiate de (a).

Le cas le plus simple est celui où $f$ est mesurable bornée :

Lemme 2.4.4. Soit $f \mathcal{B}_{d}$-mesurable bornée et soit $\varepsilon>0$; il existe alors une jauge $\gamma$ et une mesure positive $\mu$ de masse totale $\leq 1$ telles que:

$$
|f \lambda(A)-f(y) \lambda(A)| \leq 2 \varepsilon \mu(A) \text { dès que } A \subset \mathbf{U}_{d}(y, \gamma(y)), A \in \mathcal{B}_{d}
$$

(sans qu'on ait nécessairement $y \in A$ ). 
Démonstration. Par 2.4.1b, on choisit $g$ et $h \mathcal{B}{ }_{d}$-mesurables avec :

$$
\begin{aligned}
& g \text { s.c.s. de }\left(X, \mathcal{T}_{d}\right) \text { dans }[-\infty,+\infty[, \\
& \left.\left.h \text { s.c.i. de }\left(X, \mathcal{T}_{d}\right) \text { dans }\right]-\infty,+\infty\right], \\
& g \leq f \leq h \text { et } \mathbb{E}_{\lambda}(h-g)<\varepsilon
\end{aligned}
$$

Quitte à tronquer $g$ et $h$, on peut supposer que $g$ et $h$ sont bornées.

On choisit une jauge $\gamma$ telle que :

$$
g(x)-\varepsilon<g(y) \text { et } h(y)<h(x)+\varepsilon \text { pour tout } x \in \mathbf{U}_{d}(y, \gamma(y)) .
$$

Soient $y \in X$ et $A \subset \mathbf{U}_{d}(y, \gamma(y))$; on a alors pour tout $x \in A$ :

$$
g(x)-\varepsilon<g(y)<f(y)<h(y)<h(x)+\varepsilon .
$$

Comme on a $g \leq f \leq h$, on trouve $|f(x)-f(y)|<h(x)-g(x)+\varepsilon$ pour tout $x \in A$, donc $|f \lambda(A)-f(y) \lambda(A)| \leq \int_{A}(h(x)-g(x)+\varepsilon) \lambda(d x)$. On voit que $\mu=(h-g+\varepsilon) \lambda / 2 \varepsilon$ répond à la question.

Au prix de quelques efforts supplémentaires (les lemmes 2.4.5 et 2.4.6), on obtient le même résultat pour toute fonction $f$ mesurable, partout finie et $\lambda$-intégrable.

Lemme 2.4.5. Soit $\varepsilon>0$ et soit $f \mathcal{B}_{d}$-mesurable à valeurs dans $\{0\} \cup$ $\left[M,+\infty\left[(M>0)\right.\right.$ avec $\mathbb{E}_{\lambda}(f) \leq \varepsilon$. Il existe alors une jauge $\gamma$ et une mesure positive $\mu$ de masse totale $\leq 1$ telles que :

$$
f(y) \lambda(A) \leq 3 \varepsilon \mu(A) \text { dès que } A \subset \mathbf{U}_{d}(y, \gamma(y)), A \in \mathcal{B}_{d}
$$

(sans qu'on ait nécessairement $y \in A$ ).

Démonstration. On se donne des nombres $\alpha_{n}>0(n \in \mathbb{N})$ avec

$$
\sum_{n \in \mathbb{N}} M 2^{n+1} \alpha_{n} \leq \varepsilon
$$

Pour tout $n \in \mathbb{N}$, on pose $X_{n}=\left\{x \in X\left|M 2^{n} \leq\right| f(x) \mid<M 2^{n+1}\right\}$; par 2.4.1a, on peut choisir un ouvert $V_{n} \mathcal{B}_{d}$-mesurable contenant $X_{n}$ avec $\lambda\left(V_{n}\right)<\lambda\left(X_{n}\right)+\alpha_{n}$; on note $\lambda_{n}$ la mesure $A \mapsto \lambda\left(A \cap\left(V_{n} \backslash X_{n}\right)\right)$, qui est de masse totale $<\alpha_{n}$. Pour chaque $y \in X_{n}$, soit $\gamma(y)$ tel que $\mathbf{U}_{d}(y, \gamma(y)) \subset V_{n}$; pour $y \in\{f=0\}$, on prend $\gamma(y)=1$.

Soient $y \in X$ et $A \subset \mathbf{U}_{d}(y, \gamma(y))$; si $y \in\{f>0\}$, on a alors pour tout $x \in A$ et tout $n \in \mathbb{N}$ :

$$
f(y) \mathbf{I}_{X_{n}}(y) \leq 2 f(x) \mathbf{I}_{X_{n}}(x)+M 2^{n+1} \mathbf{I}_{V_{n} \backslash X_{n}}(x) .
$$


En sommant en $n \in \mathbb{N}$, on obtient pour tout $x \in A$ :

$$
f(y) \leq 2 f(x)+\sum_{n \in \mathbb{N}} M 2^{n+1} \mathbf{I}_{V_{n} \backslash X_{n}}(x) .
$$

En intégrant en $x \in A$, on obtient :

$$
f(y) \lambda(A) \leq 2 \int_{A} f(x) \lambda(d x)+\sum_{n \in \mathbb{N}} M 2^{n+1} \lambda_{n}(A) .
$$

On voit que $\mu=\frac{1}{3 \varepsilon}\left[2 f \lambda+\sum_{n \in \mathbb{N}} M 2^{n+1} \lambda_{n}\right]$ répond à la question.

Lemme 2.4.6. Soit $f \mathcal{B}_{d}$-mesurable, $\lambda$-intégrable et partout finie, et soit $\varepsilon>0$; il existe alors une jauge $\gamma$ et une mesure positive $\mu$ de masse totale $\leq 1$ telles que:

$$
|f \lambda(A)-f(y) \lambda(A)| \leq 6 \varepsilon \mu(A) \text { dès que } A \subset \mathbf{U}_{d}(y, \gamma(y))
$$

(sans qu'on ait nécessairement $y \in A$ ).

Démonstration. Soit $\varepsilon>0$; on peut écrire $f=f_{1}+f_{2}$, avec $f_{1}$ borné, $\left|f_{2}\right|$ à valeurs dans $\{0\} \cup\left[M,+\infty\left[(M>0)\right.\right.$ et $\mathbb{E}_{\lambda}\left(\left|f_{2}\right|\right) \leq \varepsilon$.

Appliquant le Lemme 2.4.4 à $f_{1}$, on trouve une jauge $\gamma_{1}$ et une mesure positive $\mu_{1}$ de masse totale $\leq 1$ telle que :

$$
\left|f_{1} \lambda(A)-f_{1}(y) \lambda(A)\right| \leq 2 \varepsilon \mu_{1}(A) \text { dès que } A \subset \mathbf{U}_{d}(y, \gamma(y)) \text {. }
$$

Appliquant le Lemme 2.4.5 à $\left|f_{2}\right|$, on trouve une jauge $\gamma_{2}$ et une mesure positive $\mu_{2}$ de masse totale $\leq 1$ telle que :

$$
\left.\left|f_{2}(y)\right| \lambda(A)\right) \leq 3 \varepsilon \mu_{2}(A) \text { dès que } A \subset \mathbf{U}_{d}(y, \gamma(y))
$$

Avec la jauge $\gamma=\gamma_{1} \wedge \gamma_{2}$, on obtient (pour $\left.A \subset \mathbf{U}_{d}(y, \gamma(y))\right)$ :

$$
\begin{aligned}
|f \lambda(A)-f(y) \lambda(A)| & \leq\left|f_{1} \lambda(A)-f_{1}(y) \lambda(A)\right|+\left|f_{2}\right| \lambda(A)+\left|f_{2}(y)\right| \lambda(A) \\
& \leq 2 \varepsilon \mu_{1}(A)+\left|f_{2}\right| \lambda(A)+3 \varepsilon \mu_{2}(A) .
\end{aligned}
$$

On voit que $\mu=\frac{1}{6 \varepsilon}\left[2 \varepsilon \mu_{1}+\left|f_{2}\right| \lambda+3 \varepsilon \mu_{2}\right]$ répond à la question.

\section{(2.5) Parties chargées par une mesure}

La propriété de dénombrabilité suivante jouera un rôle important dans la démonstration des théorèmes de dérivation aux paragraphes 3 et 4 , ainsi que ci-dessous en 2.6.

Lemme 2.5.1. Soit $X$ un ensemble muni d'une tribu $\mathcal{A}$. Soient $\nu \in$ $\mathcal{M}_{1}(X, \mathcal{A})$ et $\mathcal{F}$ une famille de parties $\mathcal{A}$-mesurables de $X$ toutes positivement chargées par $\nu$ (i.e. vérifiant $\nu(A)>0$ pour tout $A \in \mathcal{F})$. Si $\mathcal{F}$ est d'ordre $m$ fini, alors $\mathcal{F}$ est dénombrable. 
Démonstration. Soit $\varepsilon>0$; soient $A_{1}, \ldots, A_{k}$ des éléments de $\mathcal{F}$ de mesure $>\varepsilon$; on a alors :

$$
k \varepsilon<\nu\left(A_{1}\right)+\ldots+\nu\left(A_{k}\right) \leq|\nu|\left(A_{1}\right)+\ldots+|\nu|\left(A_{k}\right) \leq m|\nu|(X) ;
$$

donc le cardinal de $\{A \in \mathcal{F} \mid \nu(A)>\varepsilon\}$ est $\leq m|\nu|(X) / \varepsilon$, ce qui donne le résultat.

(2.5.2) Dans la pratique, on appliquera ce Lemme à des familles de boules fermées de $(X, d)$; de plus, la conclusion subsiste si $\nu$ est une fonction d'ensemble définie seulement sur les boules fermées de $(X, d)$ et "suradditive" au sens suivant :

il existe $c \in] 0,+\infty\left[\right.$ tel que, pour toute famille finie $\mathcal{B}=\left(B_{i}\right)_{i \in I}$ de boules fermées, on a $\sum_{i \in I} \nu\left(B_{i}\right) \leq c o(\mathcal{B})$.

\section{(2.6) Mesures portées par leur support}

On va étudier quelques cas où $Z(\lambda, d)$ est $\lambda$-négligeable (propositions 2.6.2 et 2.6.7 ci-dessous).

(2.6.1) (a) Le complémentaire de $Z(\lambda, d)$ est appelé le support de $\lambda$ relatif $\grave{a} d$; ce support est fermé dans $\left(X, \mathcal{T}_{d}\right)$ dès que chaque boule est un voisinage de son centre dans $\left(X, \mathcal{T}_{d}\right)$.

(b) Mais, en l'absence de séparabilité, il peut arriver que $\lambda$ ne soit pas portée par son support:

en effet soit $d$ la distance sur $X$ à valeurs dans $\{0,1\}$; chaque point $y$ de $X$ est une boule $\mathbf{U}_{d}(y, 1 / 2)$ et donc toute partie de $X$ est ouverte et appartient à $\mathcal{B}_{d} ;$

si le cardinal de $X$ est $U$-mesurable ("grand" cardinal au sens de Ulam, voir 2.6.4 ci-dessous), on peut trouver une mesure positive finie $\lambda$ sur $\left(X, \mathcal{B}_{d}\right)$ nulle sur les points mais non nulle (à valeurs dans $\{0,1\}$ ) ; on a alors $\lambda(Z(\lambda, d))=\lambda(X)>0$.

(c) On dit que $(X, d)$ est séparable s'il existe une partie dénombrable $D$ de $X$ rencontrant toute boule $\mathbf{U}_{d}(y, r)$.

Proposition 2.6.2. Soit $X$ un ensemble muni d'un noyau symétrique d; soit $\mathcal{A}$ une tribu sur $X$ contenant $\mathcal{A}_{d}$ et soit $\lambda \in \mathcal{M}_{1}^{+}(X, \mathcal{A})$.

(a) On suppose que d est un écart, ou plus généralement vérifie:

$d(x, y) \leq C[d(x, z)+d(z, y)]$ pour un $C \in] 0,+\infty[$ et pour tous $x, y, z \in X$.

Si $(X, d)$ est séparable, on a $\lambda^{*}(Z(\lambda, d))=0$. 
(b) On suppose que, de toute famille $\mathcal{U}$ de boules ouvertes à rayon constant, on peut extraire une famille recouvrant les centres des éléments de $\mathcal{U}$ et d'ordre fini en tout point de $X$. Si $(X, d)$ est séparable, on a $\lambda^{*}(Z(\lambda, d))=0$.

(c) On suppose que, quel que soit $r>0$, il existe un entier $m(r) \geq 1$ tel que, de toute famille $\mathcal{U}$ de boules ouvertes de rayon $r$, on peut extraire une famille recouvrant les centres des éléments de $\mathcal{U}$ et d'ordre $\leq m(r)$.

$S$ 'il existe une mesure $\mu$ positive et $\sigma$-finie chargeant toutes les boules ouvertes de $(X, d)$, on a $\lambda^{*}(Z(\lambda, d))=0$.

Démonstration. Démonstration de (a). On choisit, pour chaque $y \in Z(\lambda, d)$, une boule ouverte $\mathbf{U}_{d}\left(y, r_{y}\right)$ de rayon rationnel $r_{y}>0$ avec $\lambda\left(\mathbf{U}_{d}\left(y, r_{y}\right)\right)=0$. Soit $D$ une partie dénombrable de $X$ rencontrant toute boule ouverte.

Soit $z_{y} \in D \cap \mathbf{U}_{d}\left(y, r_{y} / 2 C\right)$; on a alors $y \in \mathbf{U}_{d}\left(z_{y}, r_{y} / 2 C\right) \subset \mathbf{U}_{d}\left(y, r_{y}\right)$.

La famille dénombrable des boules $\mathbf{U}_{d}\left(z_{y}, r_{y} / 2 C\right.$ ) (pour $y \in Z(\lambda, d)$ ) recouvre $Z(\lambda, d)$; chacune de ces boules étant $\lambda$-négligeable, on a

$$
\lambda^{*}(Z(\lambda, d))=0
$$

Démonstration de (b). On choisit, pour chaque $y \in Z(\lambda, d)$, une boule ouverte $U(y)$ de rayon rationnel $>0$ avec $\lambda(U(y))=0$. On se donne $r>0$ et on note $Z_{r}$ l'ensemble des $y \in Z(\lambda, d)$ tels que $U(y)$ soit de rayon $r$; on extrait de la famille $(U(y))_{y \in Z_{r}}$ une famille $\mathcal{U}=\left(U_{i}\right)_{i \in I}$ recouvrant $Z_{r}$ et d'ordre fini en tout point. Soit $D$ une partie dénombrable de $X$ rencontrant toute boule ouverte. Chaque élément de $\mathcal{U}$ contient un point de $D$ et chaque point de $D$ est dans un nombre fini d'éléments de $\mathcal{U}$; donc $I$ est dénombrable. Donc $\lambda^{*}\left(Z_{r}\right)=0$ pour tout $r$ rationnel ; comme les $Z_{r}$ recouvrent $Z$, on a $\lambda^{*}(Z(\lambda, d))=0$.

Démonstration de (c). On choisit, pour chaque $y \in Z(\lambda, d)$, une boule ouverte $U(y)$ de rayon dans $2^{-\mathbb{N}}$ avec $\lambda(U(y))=0$. On choisit une partition dénombrable et $\mathcal{A}$-mesurable $\left(X_{k}\right)_{k \in K}$ de $X$ telle que $\mu\left(X_{k}\right)$ soit fini pour tout $k \in K$; pour tous $k \in K$ et $A \in \mathcal{A}$, on pose $\mu_{k}(A)=\mu\left(A \cap X_{k}\right)$. On se donne $k \in K$ et on note $Z_{k}$ l'ensemble des $y \in Z(\lambda, d)$ tels que $\mu_{k}$ charge $U(y)$; on extrait de la famille $U(y)\left(y \in Z_{k}\right)$ une famille $\mathcal{U}=\left(U_{i}\right)_{i \in I}$ recouvrant $Z_{k}$ et d'ordre $\leq m(r)$.

Par 2.5.1 appliqué à $\mu_{k}, I$ est dénombrable. Donc $\lambda^{*}\left(Z_{k}\right)=0$ pour tout $k \in K$; comme les $Z_{k}$ recouvrent $Z$, on a $\lambda^{*}(Z(\lambda, d))=0$.

Dans (a), on aurait pu supposer seulement que, pour tous $y \in X$ et $r>0$, il existe $t>0$ tel que $d(x, z)<t, d(z, y)<t$ implique $d(x, y)<r$ (pour tous $x, z \in X$ ). 
(2.6.3) Soit $\alpha$ un cardinal infini ; on dit que $(X, d)$ est $\alpha$-séparable s'il existe une partie $D$ de $X$ de cardinal $\leq \alpha$ et rencontrant toute boule $\mathbf{U}_{d}(y, r)$.

Dans le reste de cette section, on va voir que, si $(X, d)$ est $\alpha$-séparable (où $\alpha$ est un "petit" cardinal au sens d'Ulam) et si $(X, d)$ vérifie une propriété de recouvrement adéquate, alors $\lambda^{*}(Z(\lambda, d))=0$.

(2.6.4) On note $\omega$ le cardinal dénombrable et $\omega^{+}$son successeur.

(a) Soit $X$ un ensemble muni d'une tribu $\mathcal{A}$. La mesure $\mu \in \mathcal{M}_{1}^{+}(X, \mathcal{A})$ est dite $\alpha$-additive (pour un $\alpha>\omega$ ) si, pour toute famille disjointe $\left(A_{j}\right)_{j \in J}$ d'éléments de $\mathcal{A}$ avec $J$ de cardinal $<\alpha, \cup_{j \in J} A_{j}$ est $\mu$-mesurable et $\mu\left(\cup_{j \in J} A_{j}\right)=\sum_{j \in J} \mu\left(A_{j}\right)$. On voit donc que toute mesure est " $\omega+$-additive" (ce qui signifie simplement " $\sigma$-additive").

(b) Supposons que la tribu $\mathcal{A}$ contienne tous les points de $X$. La mesure $\mu \in \mathcal{M}_{1}^{+}(X, \mathcal{A})$ est dite diffuse si on a $\mu(\{x\})=0$ pour tout $x \in X$.

(c) Un cardinal $\alpha>\omega$ est dit U-mesurable ou "grand" au sens d'Ulam ("messbar", dans [39, p.141]) s'il existe une mesure diffuse non nulle à valeurs dans $\{0,1\}$ sur $\left(\alpha, 2^{\alpha}\right)$; il est dit mesurable si cette mesure est $\alpha$ additive (voir [11, p. 181]). D'habitude, on ne fait pas la restriction $\alpha>\omega$, ce qui fait que $\omega$ est mesurable, mais non $U$-mesurable.

(2.6.5) Les cardinaux mesurables $>\omega$ sont exceptionnels ; si on postule leur existence et qu'on fait l'hypothèse du continu, ils sont nécessairement extrêmement grands (voir [22]). De plus le premier cardinal $U$-mesurable est mesurable (voir [22] p 297).

Voici deux résultats importants dus à Ulam [39] :

(a) Soit $\alpha \leq \omega^{+}$; alors toute mesure positive finie diffuse sur $\left(\alpha, 2^{\alpha}\right)$ est nulle.

(b) Avec l'hypothèse du continu, la même conclusion vaut pour tout cardinal $\alpha$ non $U$-mesurable (i.e. "petit" au sens d'Ulam).

Voir [22], et aussi [32, pp. 25-26] et [20, pp. 58-59].

(2.6.6) Si $d$ est un écart et $\mathcal{A} \supset \mathcal{B}_{d}$, alors toute réunion de boules ouvertes de $(X, d)$ est $\mathcal{A}$-mesurable.

De façon plus générale, soit $d$ un noyau symétrique sur un ensemble $X$ muni également d'un écart $\delta$ tel que :

- toute boule $\mathbf{U}_{d}(y, r)$ est ouverte dans $\left(X, \mathcal{T}_{\delta}\right)$,

- toute boule $\mathbf{U}_{\delta}(y, r)$ contient une boule ouverte de $(X, d)$ de même centre ;

alors toute réunion de boules ouvertes de $(X, d)$ est $\mathcal{B}_{d}$-mesurable :

en effet toute réunion de boules ouvertes de $(X, d)$ est ouverte dans $\left(X, \mathcal{T}_{\delta}\right) ;$ elle appartient donc à $\mathcal{B}_{\delta}$, donc à $\mathcal{B}_{d}\left(\right.$ car $\left.\mathcal{T}_{\delta} \subset \mathcal{T}_{d}\right)$, donc à $\mathcal{A}$. 
(c) Soit $\mathcal{A}$ une tribu sur $X$; une application $\lambda$ de $\mathcal{A}$ dans une partie bornée de $[0,+\infty[$ est appelée une $L$-mesure s'il existe $a, b \in] 0,+\infty[$ avec :

$$
a \sum_{i \in I} \lambda\left(A_{i}\right) \leq \lambda(A) \leq b \sum_{i \in I} \lambda\left(A_{i}\right)
$$

pour tout $A \in \mathcal{A}$ et toute partition finie ou dénombrable $\left(A_{i}\right)_{i \in I}$ de $A\left(A_{i} \in \mathcal{A}\right)$.

Dans la démonstration de la Proposition 2.6.7 ci-dessous, on fera usage d'une propriété des $L$-mesures dont la démonstration est reportée à 2.6.8.

Proposition 2.6.7. Soit $X$ un ensemble muni d'un noyau symétrique d ; soit $\mathcal{A}$ une tribu sur $X$ et soit $\lambda \in \mathcal{M}_{1}^{+}(X, \mathcal{A})$. On suppose que :

(a) Quel que soit $r$, il existe un entier $m(r) \geq 1$ tel que : de toute famille $\mathcal{U}$ de boules ouvertes de rayon $r$, on peut extraire une famille recouvrant les centres des éléments de $\mathcal{U}$ et d'ordre $\leq m(r)$.

(b) $(X, d)$ est $\alpha$-séparable, où $\alpha$ est un cardinal non $U$-mesurable ("petit" au sens d'Ulam);

(c) toute réunion de boules ouvertes de $(X, d)$ est $\mathcal{A}$-mesurable (par exemple d est un écart et $\mathcal{A} \supset \mathcal{B}_{d}$ ).

On a alors $\lambda^{*}(Z(\lambda, d))=0$.

Démonstration. On choisit, pour chaque $y \in Z(\lambda, d)$, une boule ouverte $U(y)$ de rayon rationnel $>0$ avec $\lambda(U(y))=0$.

On se donne $r>0$ et on note $Z_{r}$ l'ensemble des $y \in Z(\lambda, d)$ tels que $U(y)$ soit de rayon $r$; on extrait de la famille $(U(y))_{y \in Z_{r}}$ une famille $\mathcal{U}=\left(U_{k}\right)_{k \in K}$ recouvrant $Z_{r}$ et d'ordre $\leq m(r)$. Soit $D \subset X$ une partie de cardinal $\leq \alpha$ rencontrant toute boule ouverte. Chaque élément de $\mathcal{U}$ contient un point de $D$ et chaque point de $D$ est au plus dans $m(r)$ éléments de $\mathcal{U}$; donc $K$ est de cardinal $\leq \alpha$. Par $(\mathrm{c}) \cup_{k \in B} U_{k}$ appartient à $\mathcal{A}$ pour tout $B \subset K$.

On pose $\mu(B)=\lambda\left(\cup_{k \in B} U_{k}\right)$ pour tout $B \subset K$; on a donc

$$
\mu(A) \leq \sum_{i \in I} \mu\left(A_{i}\right) \leq m(r) \mu(A)
$$

pour tout $A \subset K$ et toute partition finie ou dénombrable $\left(A_{i}\right)_{i \in I}$ de $A$, autrement dit $\mu$ est une $L$-mesure sur $\left(K, 2^{K}\right)$; il résulte du Lemme 2.6.8 qu'il existe une mesure $\nu$ sur $\left(K, 2^{K}\right)$ avec $\mu \leq \nu \leq m(r) \mu$; comme on a $\lambda\left(U_{k}\right)=0$ pour tout $k \in K, \nu$ est diffuse, donc $\nu=0$ car $\alpha$ est un cardinal non $U$-mesurable (voir 2.6.5), donc $\mu=0$, donc $\lambda^{*}\left(Z_{r}\right) \leq \mu(K)=0$.

Comme les $Z_{r}$ recouvrent $Z(\lambda, d)$, on a $\lambda^{*}(Z(\lambda, d))=0$. 
Sous les hypothèses (a), (b) et (c) de 2.6.7, on peut montrer que $\lambda$ est portée par une partie mesurable séparable de $X$ :

on se donne $r>0$ et on considère la famille de toutes les boules ouvertes de rayon $r$ centrées en dehors de $Z(\lambda, d)$ (de ce fait elles sont chargées par $\lambda)$; on extrait de cette famille une famille $\mathcal{B}_{r}$ recouvrant $X \backslash Z$ et d'ordre $\leq m(r)$; par 2.6.7, $\mathcal{B}_{r}$ recouvre $\lambda$-presque tout $X$; par ailleurs, par 2.5.1, la famille $\mathcal{B}_{r}$ est dénombrable ; la mesure $\lambda$ est portée par la réunion $X_{r}$ des éléments de $\mathcal{B}_{r}$, donc par l'intersection pour tout $r$ des $X_{r}$, qui est une partie séparable de $X$.

Si $(X, d)$ est un espace métrique, on peut obtenir le même résultat sous la seule hypothèse (b) ((c) est alors automatique et on arrive à remplacer (a) par la paracompacité). De façon précise on a (voir Billingsley [8, p. 235]) :

pour que toute mesure finie sur les boréliens de l'espace métrique $(X, d)$ soit portée par une partie séparable, il faut et il suffit que toute partie discrète soit "petite" au sens d'Ulam (ce qui revient à l'hypothèse (b)).

Rappelons que, sur un espace métrique séparable complet $(X, d)$, toute mesure finie sur les boréliens est une mesure de Radon, mais que si l'espace métrique $(X, d)$ n'est pas complet, cela peut être faux (voir par exemple Billingsley [8, p. 234]) :

soit $\lambda$ la mesure de Lebesgue sur $[0,1], X$ une partie de $[0,1]$ avec $\lambda_{*}(X)<\lambda^{*}(X)$ (on munit $X$ de la distance usuelle $d$ ) ; alors $\mu: B \longmapsto$ $\lambda^{*}(B)$ (pour $B$ borélien de $(X, d)$ ) est une mesure qui n'est pas une mesure de Radon.

Lemme 2.6.8. Soit $X$ un ensemble muni d'une tribu $\mathcal{A}$ et soient $a, b \in$ ] $0,+\infty\left[\right.$. Soit $\mu$ une $L$-mesure sur $(X, \mathcal{A})$, vérifiant a $\mu(A) \leq \sum_{i \in I} \mu\left(A_{i}\right) \leq$ $b \mu(A)$ pour tout $A \in \mathcal{A}$ et toute partition finie ou dénombrable $\left(A_{i}\right)_{i \in I}$ de $A$ $\left(A_{i} \in \mathcal{A}\right)$. Alors il existe une mesure $\nu$ finie $\operatorname{sur}(X, \mathcal{A})$ avec $a \mu \leq \nu \leq b \mu$.

Démonstration. Soit $\Pi$ l'ensemble des partitions finies ou dénombrables $\mathcal{A}$-mesurables de $X$. Soient $\pi=\left(A_{i}\right)_{i \in I}$ et $\rho=\left(B_{j}\right)_{j \in J}$ deux éléments de $\Pi$; on dit que $\pi$ est plus fine que $\rho$ (noté $\pi \succ \rho$ ) si tout $A_{i}$ est contenu dans un $B_{j}$.

Soit $D$ un ultrafiltre sur $\Pi$ contenant $\{\pi \in \Pi \mid \pi \succ \rho\}$ pour tout $\rho \in \Pi$.

Si $\pi=\left(A_{i}\right)_{i \in I}$ est un élément de $\Pi$, on pose $\mu_{\pi}(A)=\sum_{i \in I} \mu\left(A \cap A_{i}\right)$; on note que $\mu_{\pi}$ est une $L$-mesure vérifiant $a \mu \leq \mu_{\pi} \leq b \mu$.

On pose $\nu(A)=\lim _{\pi, D} \mu_{\pi}(A)$; on a $a \mu \leq \nu \leq b \mu$; on va montrer que $\nu$ est une mesure. 
(a) Soient $A \in \mathcal{A}$ et $\left(A_{1}, \ldots, A_{n}\right)$ une partition finie $\mathcal{A}$-mesurable de $A$; on se donne $\rho \in \Pi$ contenant chacun des $A_{i}$; on a alors :

$$
\begin{aligned}
& \mu_{\pi}(A)=\mu_{\pi}\left(A_{1}\right)+\ldots+\mu_{\pi}\left(A_{n}\right), \text { pour tout } \pi \succ \rho \\
& \text { (car, si } \left.\pi=\left(P_{k}\right)_{k \in K}, \text { chaque } A_{i} \text { est une réunion de } P_{k}\right) .
\end{aligned}
$$

On a donc $\nu(A)=\nu\left(A_{1}\right)+\ldots+\nu\left(A_{n}\right)$.

(b) Soient $A \in \mathcal{A}$ et $\left(A_{i}\right)_{i \in \mathbb{N}}$ une partition $\mathcal{A}$-mesurable de $A$; soit $\varepsilon>0$, il existe $p \in \mathbb{N}$ tel que $\sum_{i>p} \mu\left(A_{i}\right) \leq \varepsilon$, donc $\mu\left(\cup_{i \geq p} A_{i}\right) \leq \varepsilon / a$; on a donc $\sum_{i>p} \nu\left(A_{i}\right) \leq b \varepsilon$ et $\nu\left(\cup_{i \geq p} A_{i}\right) \leq b \varepsilon / a$; au vu de (a), on en déduit que $\left|\nu\left(\cup_{i \in \mathbb{N}} A_{i}\right)-\sum_{i \in \mathbb{N}} \nu\left(A_{i}\right)\right| \leq b \varepsilon+b \varepsilon / a$; comme $\varepsilon$ est arbitraire, $\nu$ est une mesure.

(2.6.9) Inversement, soient $\left.\nu \in \mathcal{M}_{1}^{+}(X, \mathcal{A}), a, b \in\right] 0,+\infty[$ et $\mu: \mathcal{A} \longrightarrow$ $[0,+\infty[$ avec $a \nu(A) \leq \mu(A) \leq b \nu(A)$ pour tout $A \in \mathcal{A}$; on voit facilement que $\mu$ est une $L$-mesure.

Les propriétés de recouvrement concernant les boules ouvertes qui sont utilisées en 2.6.2 et 2.6.7 sont des conséquences de la propriété de recouvrement faible pour les familles de boules fermées à rayon constant.

Lemme 2.6.10. Soit $m$ un entier $\geq 1$. On suppose que, de toute famille $\mathcal{B}$ de boules fermées à rayon constant, on peut extraire une famille recouvrant les centres des éléments de $\mathcal{B}$ et d'ordre $\leq m$. Alors, de toute famille $\mathcal{U}$ de boules ouvertes à rayon constant, on peut extraire une famille recouvrant les centres des éléments de $\mathcal{U}$ et d'ordre $\leq m$.

Démonstration. Soit $\mathcal{U}=\left(\mathbf{U}_{d}(y, r)\right)_{y \in T}$ une famille de boules ouvertes de rayon $r$; on extrait de $T$ une partie maximale $S$ dont les points soient d'éloignements mutuels $\geq r$.

Soit $z \in X$ et soit $R$ une partie finie de $S$ telle que $z \in \mathbf{U}_{d}(y, r)$ pour tout $y \in R$. On choisit $\varepsilon>0$ tel que $z \in \mathbf{B}_{d}(y, r-\varepsilon)$ pour tout $y \in R$.

Soit $\mathcal{B}=\left(\mathbf{B}_{d}(y, r-\varepsilon)\right)_{y \in R} ;$ le cardinal de $R$ est $\leq m$ :

en effet, on peut extraire de $\mathcal{B}$ une famille $\mathcal{B}^{\prime}$ recouvrant les centres des éléments de $\mathcal{B}$ (donc égale à $\mathcal{B}$ car $y^{\prime} \notin \mathbf{B}_{d}\left(y, r-\varepsilon\right.$ ) pour tous $y, y^{\prime} \in R$ distincts) et d'ordre $\leq m$ (donc de cardinal $\leq m$ car $z \in \mathbf{B}_{d}(y, r-\varepsilon$ ) pour tout $y \in R$ ).

Donc la famille $\left(\mathbf{U}_{d}(y, r)\right)_{y \in S}$, qui recouvre $T$, est d'ordre $\leq m$.

On montrerait aisément la réciproque de ce lemme. De même, on l'étendrait sans difficulté aux familles à rayons discrets, ce qui fait que l'essentiel des paragraphes 3 et 4 s'étend aux boules ouvertes. 


\section{(2.7) Mesurabilité}

Avec des hypothèses convenables (satisfaites si $d$ est un écart), on peut montrer que $T_{r}(\nu, \lambda)$ et $Z(\lambda, d)$ sont $\mathcal{B}_{d}$-mesurables. On ne se servira pas de cela dans la suite, préférant travailler avec des intégrales supérieures, ce qui sera plus général bien qu'amenant quelques complications.

(2.7.1) Soit $X$ muni d'un noyau symétrique $d$ et d'une tribu $\mathcal{A}$. On dit que $(X, d)$ est fortement $\mathcal{A}$-mesurable si les applications $y \mapsto \lambda\left(\mathbf{U}_{d}(y, r)\right)$ et $y \mapsto \lambda\left(\mathbf{B}_{d}(y, r)\right)$ sont $\mathcal{A}$-mesurables pour tout $\lambda \in \mathcal{M}_{1}^{+}(X, \mathcal{A})$ et tout $r>0$.

Il est clair que $y \mapsto \lambda\left(\mathbf{B}_{d}(y, r)\right)$ est $\mathcal{A}$-mesurable dès que $y \mapsto \lambda\left(\mathbf{U}_{d}(y, r)\right)$ l'est, et inversement.

Si $(X, d)$ est fortement $\mathcal{A}$-mesurable, on a alors :

(a) $Z(r)=\left\{y \in X \mid \lambda\left(\mathbf{B}_{d}(y, r)\right)=0\right\}$ est $\mathcal{A}$-mesurable ;

(b) la fonction $y \mapsto T_{r}(\nu, \lambda)(y)$ est $\mathcal{A}$-mesurable :

en effet, soit $Y(r)$ la réunion de $\left\{y \in X \mid \lambda\left(\mathbf{B}_{d}(y, r)\right)>0\right\}$ et de $\{y \in$ $\left.X \mid \nu\left(\mathbf{B}_{d}(y, r)\right)>0\right\}$, qui sont $\mathcal{A}$-mesurables par (a) ; pour $y \notin Y(r)$, on a $T_{r}(\nu, \lambda)(y)=0$; pour $y \in Y(r), T_{r}(\nu, \lambda)(y)$ est le quotient de $\nu\left(\mathbf{B}_{d}(y, r)\right)$ par $\lambda\left(\mathbf{B}_{d}(y, r)\right)$; donc $T_{r}(\nu, \lambda)$ est $\mathcal{A}$-mesurable ;

(c) la fonction $y \mapsto \sup _{0<u<r} T_{u}(\nu, \lambda)(y)$ est $\mathcal{A}$-mesurable :

en effet, soient $Y=\cup_{n \in \mathbb{N}} Y\left(r-2^{-n}\right)$ et $S_{r}=\sup _{0<u<r} T_{u}(\nu, \lambda)$; pour $y \notin Y$, on a $S_{r}(y)=0$; pour $y \in Y$, on a $S_{r}(y)=\sup _{0<u<r, u \in \mathbb{Q}} T_{u}(\nu, \lambda)(y)$, car $u \mapsto T_{u}(\nu, \lambda)(y)$ est continue à droite pour $u \geq r-2^{-n}$ si $y \in Y\left(r-2^{-n}\right)$.

(d) la fonction $y \mapsto \sup _{u \in U} T_{u}(\nu, \lambda)(y)$ est $\mathcal{A}$-mesurable, dès que $U$ est un ouvert de $] 0,+\infty[$ (ce qu'on montrerait comme en $(c)$ ).

Lemme 2.7.2. Soit $X$ un ensemble muni d'un noyau symétrique d et d'une tribu $\mathcal{A}$. L'espace $(X, d)$ est fortement $\mathcal{A}$-mesurable dès qu'on se trouve dans l'un des cinq cas suivants :

(i) $x, y \mapsto d(x, y)$ est $\mathcal{A} \times \mathcal{A}$-mesurable ;

(ii) toute réunion de boules ouvertes de $(X, d)$ est $\mathcal{A}$-mesurable et, pour tous $y \in X, r>0$ et $\varepsilon>0$, il existe $\eta>0$ tel que $\mathbf{U}_{d}(z, r) \supset$ $\mathbf{U}_{d}(y, r-\varepsilon)$ pour tout $z \in \mathbf{U}_{d}(y, \eta)$;

(iii) toute réunion de boules ouvertes de $(X, d)$ est $\mathcal{A}$-mesurable et, pour tous $y \in X, r>0$ et $\varepsilon>0$, il existe $\eta>0$ tel que $\mathbf{B}_{d}(z, r) \subset$ $\mathbf{B}_{d}(y, r+\varepsilon)$ pour tout $z \in \mathbf{U}_{d}(y, \eta)$; 
(iv) le noyau d vérifie :

$$
|d(x, z)-d(x, y)| \leq \varphi(d(y, z)) \text { pour tous } x, y, z \in X
$$

(où $\varphi$ est une application nulle et continue en 0 ).

(v) d est un écart et $\mathcal{A} \supset \mathcal{B}_{d}$.

Démonstration. (i) Par le théorème de Fubini (voir [14, p. 31]), la fonction $y \mapsto \lambda\left(\mathbf{B}_{d}(y, r)\right)$ est $\mathcal{A}$-mesurable quel que soit $\lambda \in \mathcal{M}_{1}^{+}(X, \mathcal{A})$.

(ii) Pour tout $\alpha \geq 0$, l'ensemble $V=\left\{y \in X \mid \lambda\left(\mathbf{U}_{d}(y, r)\right)>\alpha\right\}$ est une réunion de boules ouvertes, donc $\mathcal{A}$-mesurable :

en effet $y \in V$; on fixe $\varepsilon>0$ assez petit pour qu'on ait $\lambda\left(\mathbf{U}_{d}(y, r-\varepsilon)\right)>\alpha$; on fixe $\eta>0$ tel que $\mathbf{U}_{d}(z, r) \supset \mathbf{U}_{d}(y, r-\varepsilon)$ pour tout $z \in \mathbf{U}_{d}(y, \eta)$; on a donc $\mathbf{U}_{d}(y, \eta) \subset V$.

(iii) Pour tout $\alpha \geq 0$, l'ensemble $V=\left\{y \in X \mid \lambda\left(\mathbf{B}_{d}(y, r)\right)<\alpha\right\}$ est une réunion de boules ouvertes, donc $\mathcal{A}$-mesurable :

en effet $y \in V$; on fixe $\varepsilon>0$ assez petit pour qu'on ait $\lambda\left(\mathbf{B}_{d}(y, r+\varepsilon)\right)<\alpha$; on fixe $\eta>0$ tel que $\mathbf{B}_{d}(z, r) \subset \mathbf{B}_{d}(y, r+\varepsilon)$ pour tout $z \in \mathbf{U}_{d}(y, \eta)$; on a donc $\mathbf{U}_{d}(y, \eta) \subset V$.

(iv) et (v) L'un et l'autre sont des cas particuliers de (ii) et (iii).

(2.7.3) (a) Noter que, si $(X, d)$ est un espace métrique séparable, et si on munit $X^{2}$ d'une distance $d_{2}$ définissant la topologie produit, alors la tribu de Baire $\mathcal{B}_{d_{2}}$ est la tribu produit $\mathcal{B}_{d} \times \mathcal{B}_{d}$ (ce n'est pas le cas en général, voir [23]) ; alors $\left\{(x, y) \in X^{2} \mid d(x, y) \leq r\right\}$ appartient à $\mathcal{B}_{d} \times \mathcal{B}_{d}$ (car $d$ est continue sur $\left.X^{2}\right)$.

(b) Soit $(X, \delta)$ un espace métrique séparable (donc $\mathcal{A}_{\delta}=\mathcal{B}_{\delta}$ ) ; si $d$ est bi-Lipschitz équivalent à $\delta\left(\right.$ donc $\left.\mathcal{B}_{d}=\mathcal{B}_{\delta}\right)$ et que $\mathcal{A}_{d}$ est inclus dans $\mathcal{B}_{\delta}$, alors $\mathcal{A}_{d}=\mathcal{B}_{\delta}$.

\section{Dérivation des mesures (convergence en mesure)}

L'objectif de ce paragraphe est d'arriver à la dérivation en mesure des mesures par les étapes mentionnées dans l'introduction. Les résultats principaux se trouvent en 3.1 (intégrabilité du quotient de dérivation) et en 3.3 (dérivation en mesure).

On montre que $T_{r}(\nu, \lambda)$ est $\lambda$-intégrable pour tous $\lambda, \nu$ si et seulement si $(X, d)$ possède, pour un $m$ entier $\geq 1$, la propriété de recouvrement faible de degré $\leq m$ restreinte aux boules de rayon $r$ :

de toute famille $\mathcal{B}$ de boules fermées de rayon $r$, on peut extraire une famille recouvrant les centres des éléments de $\mathcal{B}$ et d'ordre $\leq m$ (pour les boules ouvertes cette propriété a déjà été utilisée en 2.6.2b et 2.6.7). 
Pour arriver à un théorème de dérivation en mesure, on doit supposer que $(X, d)$ possède la propriété précédente pour tout $r$ pour un $m$ indépendant de $r$, c'est à dire que $(X, d)$ possède la propriété de recouvrement faible de degré $\leq m$ restreinte aux familles de boules fermées à rayon constant; cela signifie exactement que $\mathbf{n}_{C} \leq m$ (voir 2.2a) et aussi que la dimension de De Groot de $(X, d)$ est $\leq m-1$ (voir plus bas en 3.5). Mais des hypothèses de mesurabilité supplémentaires sont nécessaires (voir 3.2).

Au lieu de supposer que $(X, d)$ vérifie la propriété de recouvrement faible de degré $\leq m$ restreinte aux familles de boules fermées à rayon constant, on supposera généralement dans ce paragraphe que $(X, d)$ vérifie seulement la propriété de recouvrement faible de degré $\leq m$ restreinte aux familles de boules fermées centrées dans $Y$ à rayon constant (en bref $\operatorname{prf}(m)$ pour les boules centrées dans $Y$ à rayon constant), où $Y$ est une partie de $X$.

Proposition 3.1. Soit $X$ un ensemble muni d'un noyau symétrique quelconque $d$, et soit $Y \subset X ;$ soit $\mathcal{A}$ une tribu sur $X$ avec $\mathcal{A} \supset \mathcal{A}_{d}$. Soient $r>0$ et $m$ un entier $\geq 1$. Alors les assertions suivantes sont équivalentes :

(g1) pour tous $a \in X$ et tous $y_{0}, \ldots, y_{m} \in Y \cap \mathbf{B}_{d}(a, r)$, il existe $i, j$ distincts avec $d\left(y_{i}, y_{j}\right) \leq r$.

(g2) $(X, d)$ possède la $\operatorname{prf}(m)$ restreinte aux boules centrées dans $Y$ et de rayon $r$;

(g3) pour tout $\lambda \in \mathcal{P}(X, \mathcal{A})$ et tout $\nu \in \mathcal{M}_{1}^{+}(X, \mathcal{A})$, on a :

$$
\mathbb{E}_{\lambda}^{*}\left(T_{r}(\nu, \lambda) \mathbf{I}_{Y}\right) \leq m \nu(X) .
$$

Démonstration. Démonstration de $(g 1) \Longrightarrow(g 2)$. Soit $T$ une partie de $Y$ et soit $\mathcal{B}=\left\{\mathbf{B}_{d}(y, r)\right\}_{y \in T}$; soit $Z$ une partie maximale de $T$ telle que $d\left(z, z^{\prime}\right)>r$ pour tous $z, z^{\prime}$ distincts dans $Z$. Alors les $\mathbf{B}_{d}(z, r)(z \in Z)$ recouvrent $T$; de plus on a $z \notin \mathbf{B}_{d}\left(z^{\prime}, r\right)$ pour tous $z, z^{\prime}$ distincts dans $Z$.

La famille des $\mathbf{B}_{d}(z, r)(z \in Z)$ est d'ordre $\leq m$ dans $X$ :

en effet soient $a \in X$ et $z_{1}, \ldots, z_{k} \in Z$ vérifiant $a \in \mathbf{B}_{d}\left(z_{i}, r\right)$ pour tous $i \in\{1, \ldots, k\}$; or on a $d\left(z_{i}, z_{j}\right)>r$ pour tous $i, j$ distincts dans $\{1, \ldots, k\}$; donc (g1) implique $k \leq m$.

Démonstration de $\left(\right.$ g2) $\Longrightarrow\left(\right.$ g3). Soient $\lambda \in \mathcal{P}(X, \mathcal{A})$ et $\nu \in \mathcal{M}_{1}^{+}(X, \mathcal{A})$; posons $T_{r}(y)=T_{r}(\nu, \lambda)(y)$ pour chaque $y \in X$. On va faire le calcul en quatre étapes :

(1) On fixe $p$ entier $>0$ et on pose $Y_{p}=Y \cap\left\{0<T_{r} \leq p\right\}$. On fixe $\tau>1$ et on pose

$$
Y_{p, n}=Y \cap\left\{p \tau^{-(n+1)}<T_{r} \leq p \tau^{-n}\right\}
$$

pour tout $n \in \mathbb{N}$. 
On choisit une famille maximale $Z_{p, 0}$ de points de $Y_{p, 0}$ d'éloignements mutuels $>r($ pour $d)$; si on a choisi $Z_{p, 0}, \ldots, Z_{p, n-1}$, on choisit une famille maximale $Z_{p, n}$ de points de $Y_{p, n} \backslash \cup\left\{\mathbf{B}_{d}(y, r) \mid y \in \cup_{0 \leq k<n} Z_{p, k}\right\}$ d'éloignements mutuels $>r$ (pour $d$ ). Notons $\left(y_{i}\right)_{i \in I}$ la famille de tous les points de $\cup_{n \in \mathbb{N}} Z_{p, n}$; on munit $I$ d'un bon ordre de façon que $y_{i} \in Z_{p, k}, y_{j} \in Z_{p, k^{\prime}}$, $k<k^{\prime}$ implique $i<j$. On pose $B_{i}=\mathbf{B}_{d}\left(y_{i}, r\right)$ pour tout $i \in I$.

On a alors :

(i) $d\left(y_{i}, y_{j}\right)>r$ pour tous $i, j \in I$ distincts ;

(ii) pour tout $y \in Y_{p}$, il existe $j \in I$ avec $d\left(y, y_{j}\right) \leq r$ et $T_{r}(y) \leq \tau T_{r}\left(y_{j}\right)$;

(iii) pour tous $i, j \in I, j \geq i$, on a $T_{r}\left(y_{j}\right) \leq \tau T_{r}\left(y_{i}\right)$.

Par (g2), on extrait de la famille $\left(B_{i}\right)_{i \in I}$, une sous-famille $\left(B_{i}\right)_{i \in J}$ recouvrant les centres de la première et d'ordre $\leq m$; à cause de (i), on a $J=I$; donc la famille $\left(B_{i}\right)_{i \in I}$ est d'ordre $\leq m$; comme $\nu$ charge tous les $B_{i}$, l'ensemble $I$ est dénombrable par 2.5.1.

De plus, par (ii), les ensembles $B_{i}(i \in I)$ recouvrent $Y_{p}$; comme $I$ est bien ordonné, les ensembles $A_{i}=B_{i} \backslash \cup_{k<i} B_{k}(i \in I)$ recouvrent $Y_{p}$.

Soit $y \in A_{i} \cap Y_{p}$; par (ii), il existe $j \in I$ avec $d\left(y, y_{j}\right) \leq r$ (i.e. $y \in B_{j}$ ) et $T_{r}(y) \leq \tau T_{r}\left(y_{j}\right)$; comme $y$ est dans $A_{i}$, il n'est dans aucun des $B_{k}$ pour $k<i$; comme $y$ est dans $B_{j}$, on a $j \geq i$; par (iii), on a donc $T_{r}(y) \leq \tau^{2} T_{r}\left(y_{i}\right)$.

Cela donne :

$$
\begin{aligned}
\mathbb{E}_{\lambda}^{*}\left(T_{r} \mathbf{I}_{Y_{p}}\right) & \leq \sum_{i \in I} \mathbb{E}_{\lambda}^{*}\left(T_{r} \mathbf{I}_{A_{i} \cap Y_{p}}\right) \leq \tau^{2} \sum_{i \in I} T_{r}\left(y_{i}\right) \lambda\left(A_{i} \cap Y_{p}\right) \\
& \leq \tau^{2} \sum_{i \in I} T_{r}\left(y_{i}\right) \lambda\left(B_{i}\right)=\tau^{2} \sum_{i \in I} \nu\left(B_{i}\right) \leq m \tau^{2} \nu(X) .
\end{aligned}
$$

En faisant tendre $\tau$ vers 1 , on obtient $\mathbb{E}_{\lambda}^{*}\left(T_{r} \mathbf{I}_{Y_{p}}\right) \leq m \nu(X)$.

On pose $Y^{\prime}=Y \cap\left\{0<T_{r}<+\infty\right\}$; faisant tendre $p$ vers $+\infty$, on obtient $\mathbb{E}_{\lambda}^{*}\left(T_{r} \mathbf{I}_{Y^{\prime}}\right) \leq m \nu(X)$.

(2) Soit $Y_{0}=Y \cap\left\{T_{r}=0\right\}$; on a évidemment $\mathbb{E}_{\lambda}^{*}\left(T_{r} \mathbf{I}_{Y_{0}}\right)=0$.

(3) Enfin, soit $\left\{y_{i}\right\}_{i \in I}$ une famille maximale de points de $Y_{\infty}=\left\{T_{r}=\right.$ $+\infty\}$ d'éloignements mutuels $>r($ pour $d)$.

Par (g2), la famille des boules $\left(B_{i}\right)_{i \in I}$ est d'ordre $\leq m$; comme $\nu$ charge tous les $B_{i}$, l'ensemble $I$ est dénombrable par 2.5.1.

Les ensembles $B_{i}(i \in I)$ recouvrent $Y_{\infty}$; comme ces ensembles sont de mesure nulle pour $\lambda$, on a $\lambda^{*}\left(Y_{\infty}\right)=0$.

(4) Par (1), (2) et (3), on trouve $\mathbb{E}_{\lambda}^{*}\left(T_{r} \mathbf{I}_{Y}\right) \leq m \nu(X)$, ce qui est le résultat demandé. 
Démonstration de $(g 3) \Longrightarrow(g 1)$. Soient $a \in X$ et $y_{0}, \ldots, y_{m} \in Y \cap$ $\mathbf{B}_{d}(a, r)$; On pose $\mathbf{B}_{d}\left(y_{i}, r\right)=B_{i}$; soit $\lambda=\frac{1}{m+1}\left(\delta_{y_{0}}+\ldots+\delta_{y_{m}}\right)$ et soit $\nu=\delta_{a}$. En appliquant (g3) et l'inégalité de Tchebychev avec $\alpha=m+\frac{1}{2}$, on obtient $\lambda\left(\left\{T_{r}(\nu, \lambda)>m+\frac{1}{2}\right\}\right)<1$; il existe donc $i$ tel que $T_{r}(\nu, \lambda)\left(y_{i}\right) \leq m+\frac{1}{2}$, c'est à dire $\nu\left(B_{i}\right) / \lambda\left(B_{i}\right) \leq m+\frac{1}{2}$; comme $\nu\left(B_{i}\right)=1$ (car $\left.a \in B_{i}\right)$, on a donc :

cardinal $\left\{j \mid 0 \leq j \leq m, d\left(y_{i}, y_{j}\right) \leq r\right\}>\left(m+\frac{1}{2}\right) \lambda\left(\mathbf{B}_{d}\left(y_{i}, r\right)\right) \geq 1 ;$

donc il existe $j \neq i$ avec $d\left(y_{i}, y_{j}\right) \leq r$.

L'argument principal de 3.1 est inspiré de la preuve d'un résultat de Mattila [26].

Si on remplace $\mathbb{E}_{\lambda}^{*}\left(T_{r}(\nu, \lambda) \mathbf{I}_{Y}\right) \leq m \nu(X)$ par $\alpha \lambda^{*}\left(Y \cap\left\{T_{r}(\nu, \lambda)>\alpha\right\}\right) \leq$ $m \nu(X)$ (pour tout $\alpha>0$ ), le théorème 3.1 est beaucoup plus facile à démontrer ; cette version affaiblie suffirait à démontrer la convergence en mesure dans 3.3.1.

\section{(3.2) Convergences}

(3.2.1) Soient $h_{r}: X \longrightarrow \overline{\mathbb{R}}$ (pour $r>0$ ) et $h: X \longrightarrow \overline{\mathbb{R}}$. Soit $Y$ une partie de $X$. On va voir quelles difficultés se posent, en l'absence de mesurabilité, pour la convergence en mesure et la convergence dans $L^{1}$.

(a) (convergence presque-sûre sur $Y$ )

On dit que $h_{r}$ tend vers $h \lambda$-presque sûrement sur $Y$ lorsque $r$ tend vers 0 si $\lambda^{*}\left(Y \cap\left\{\lim \sup _{r \longrightarrow 0}\left|h_{r}-h\right|>0\right\}\right)=0$.

(b) (convergence en mesure sur $Y$ )

On dit que $h_{r}$ tend vers $h$ en mesure sur $Y$ (relativement à $\lambda$ ) lorsque $r$ tend vers 0 si $\lim \sup _{r \longrightarrow 0} \lambda^{*}\left(Y \cap\left\{\left|h_{r}-h\right|>\alpha\right\}\right)=0$ pour tout $\alpha>0$.

Si $Y$ et $h_{r}$ sont mesurables, la convergence presque-sûre implique la convergence en mesure ; mais en l'absence de mesurabilité (même si $Y=X$ ), c'est faux (voir [40] p. 13).

(c) (convergence dans $L^{1}$ sur $Y$ )

On dit que $h_{r}$ tend vers $h$ dans $L^{1}(Y, \mathcal{A}, \lambda)$ lorsque $r$ tend vers 0 si $\mathbb{E}_{\lambda}^{*}\left(\left|h_{r}-h\right| \mathbf{I}_{Y}\right)$ tend vers 0 lorsque $r$ tends vers 0 .

La convergence en mesure et l'équi-intégrabilité impliquent la convergence dans $L^{1}$; mais en l'absence de mesurabilité (même si $Y=X$ ), la convergence presque-sûre et l'équi-intégrabilité n'impliquent pas la convergence dans $L^{1}$ (voir encore [40, p. 13]). 
(3.2.2) Lorsque $g$ est continue bornée sur $\left(X, \mathcal{T}_{d}\right), T_{r}(g \lambda, \lambda)$ tend vers $g$ en tout point de $X \backslash Z(\lambda, d)$ quand $r$ tend vers 0 ; néanmoins, on n'est pas assuré en général, faute de mesurabilité, que $\lambda^{*}\left((X \backslash Z(\lambda, d)) \cap\left\{\left|T_{r}(g \lambda, \lambda)-g\right|>\alpha\right\}\right)$ tende vers 0 . Pour avoir cette conclusion, on va se placer dans un des deux cas suivants :

(a) (cas non séparable avec des boules ouvertes)

toute boule ouverte de $(X, d)$ est ouverte dans $\left(X, \mathcal{T}_{d}\right)$, et tout ouvert de $\left(X, \mathcal{T}_{d}\right)$ est $\mathcal{A}$-mesurable (c'est le cas si $d$ est un écart) ;

(b) (cas séparable avec des boules mesurables)

(X,d) est séparable (voir 2.6.1), on a $\mathcal{A}_{d} \subset \mathcal{B}_{d} \subset \mathcal{A}$ et il existe $\theta: r \mapsto$ $\theta(r)>0$ et $\eta: y, r \mapsto \eta(y, r)>0$ tels que $d(y, x)<\theta(r), d(x, z)<\eta(y, r)$ implique $d(y, z)<r$ pour tout $x, y, z \in X$ et tout $r>0$.

(c'est le cas si on a $d(x, y) \leq C[d(x, z)+d(z, y)]$ pour un $C \in] 0,+\infty[$ et tous $x, y, z$, en particulier si $d$ est bi-Lipschitz équivalent à une distance).

Lemme 3.2.3. Soit $X$ muni d'un noyau symétrique $d$ et d'une tribu $\mathcal{A}$. Soit $\alpha>0$. Soit $g$ une application $\mathcal{A}$-mesurable continue de $\left(X, \mathcal{T}_{d}\right)$ dans $\mathbb{R}$. On pose $\omega_{r}(g)(y)=\sup \left\{|g(x)-g(y)| \mid x \in \mathbf{U}_{d}(y, r)\right\}$.

Alors, pour tout $\lambda \in \mathcal{P}(X, \mathcal{A})$ et tout $r>0,(X \backslash Z(\lambda, d)) \cap\left\{\mid T_{r}(g \lambda, \lambda)-\right.$ $g \mid>\alpha\}$ est inclus dans $G_{r, \alpha}=\left\{y \in X \mid \omega_{2 r}(g)(y)>\alpha\right\}$, qui décroît vers $\varnothing$ lorsque $r$ décrô̂t vers 0 .

De plus, $\lim _{r \longrightarrow 0} \lambda^{*}\left(G_{r, \alpha}\right)=0$ pour tout $\lambda \in \mathcal{P}(X, \mathcal{A})$ dès que $(X, d)$ vérifie 3.2.2a (c'est le cas si d est un écart) ou 3.2.2b.

Démonstration. On a $\left|T_{r}(g \lambda, \lambda)(y)-g(y)\right| \leq \omega_{2 r}(g)(y)$ pour tout $y \in$ $X \backslash Z(\lambda, d)$; de plus, comme $g$ est continue, $\omega_{2 r}(g)(y)$ tend vers 0 lorsque $r$ tend vers 0 , pour tout $y \in X$; donc $G_{r, \alpha}$ décroît vers $\varnothing$ lorsque $r$ décroît vers 0 .

On se place maintenant dans un des cas 3.2.2a ou 3.2.2b :

(a) on a $G_{r, \alpha}=\cup_{x \in X}\left(\mathbf{U}_{d}(x, 2 r) \cap\{y \in X|| g(x)-g(y) \mid>\alpha\}\right) ; G_{r, \alpha}$ est donc ouvert dans $\left(X, \mathcal{T}_{d}\right)$, donc $\mathcal{A}$-mesurable ; donc $\lim _{r \longrightarrow 0} \lambda\left(G_{r, \alpha}\right)=0$;

(b) soit $D$ une partie dénombrable de $X$ qui coupe toute boule ouverte de $(X, d)$; or on a pour tout $r>0$ et tout $t \in] 0, \theta(2 r) / 2]$ :

$G_{t, 2 \alpha} \subset G_{r, \alpha}^{\prime}=\cup_{x \in D}\left(\mathbf{U}_{d}(x, 2 r) \cap\{y \in X|| g(x)-g(y) \mid>\alpha\}\right) \subset G_{r, \alpha}$

(ce que l'on montrera un peu plus loin);

comme $G_{r, \alpha}^{\prime}$ est $\mathcal{A}$-mesurable, on a $\lim _{t \longrightarrow 0} \lambda^{*}\left(G_{t, 2 \alpha}\right) \leq \lim _{r \longrightarrow 0} \lambda\left(G_{r, \alpha}^{\prime}\right)=0$.

Il reste à montrer que $G_{t, 2 \alpha} \subset G_{r, \alpha}^{\prime}$ pour $\left.\left.t \in\right] 0, \theta(2 r) / 2\right]$. Soit $y$ un point de $G_{t, 2 \alpha}$; il existe $x \in X$ avec $d(y, x)<2 t \leq \theta(2 r)$ et $|g(x)-g(y)|>$ $2 \alpha$; on choisit $x^{\prime} \in D$ assez proche de $x$ pour avoir $d\left(x, x^{\prime}\right)<\eta(y, 2 r)$ et $\left|g\left(x^{\prime}\right)-g(x)\right| \leq \alpha$, donc $d\left(y, x^{\prime}\right)<2 r$ et $\left|g\left(x^{\prime}\right)-g(y)\right|>\alpha$. 
Pour démontrer le théorème de dérivation en mesure dans le cas de deux mesures étrangères, on a besoin de savoir que $\lambda^{*}(\{y \in X \mid 0<d(y, F)<r\})$ tend vers 0 quand $r$ tend vers 0 (pour tout $F$ fermé $\mathcal{A}$-mesurable) ; c'est le cas lorsque $(X, d)$ vérifie $3.2 .2 \mathrm{a}$ ou $3.2 .2 \mathrm{~b}$ :

Lemme 3.2.4. Soit $X$ muni d'un noyau symétrique $d$ et d'une tribu $\mathcal{A}$.

Soit $F$ un fermé $\mathcal{A}$-mesurable de $\left(X, \mathcal{T}_{d}\right)$. On posed $(y, F)=\inf \{d(y, z) \mid$ $z \in F\}$ et $W_{r}=\{y \in X \mid 0<d(y, F)<r\}$.

Il est clair que $W_{r}$ décrô̂t vers $\varnothing$ lorsque $r$ décrô̂t vers 0 .

De plus $\lim _{r \longrightarrow 0} \lambda^{*}\left(W_{r}\right)=0$ pour tout $\lambda \in \mathcal{P}(X, \mathcal{A})$ dès que $(X, d)$ vérifie $3.2 .2 \mathrm{a}$ (c'est le cas si d est un écart) ou 3.2.2b.

Démonstration. On pose $V=X \backslash F$ et on note que $W_{r}$ décroît vers $\varnothing$ quand $r$ décroît vers 0 . On distingue maintenant les cas $3.2 .2 \mathrm{a}$ et $3.2 .2 \mathrm{~b}$ :

(a) on a $W_{r}=\cup_{x \in F}\left(\mathbf{U}_{d}(x, r) \cap V\right) ; W_{r}$ est donc ouvert dans $\left(X, \mathcal{T}_{d}\right)$, donc $\mathcal{A}$-mesurable; on a donc $\lim _{r \longrightarrow 0} \lambda\left(W_{r}\right)=0$;

(b) soit $D$ une partie dénombrable de $X$ qui coupe toute boule ouverte de $(X, d)$; on choisit une partie dénombrable $E$ de $F$ qui coupe toute boule ouverte centrée dans $D$ et coupant $F$; or on a pour tout $r>0$ et tout $t \in] 0, \theta(r)]$ :

$$
W_{t} \subset W_{r}^{\prime}=\cup_{x \in E}\left(\mathbf{U}_{d}(x, r) \cap V\right) \subset W_{r}
$$

(ce que l'on montrera un peu plus loin) ; comme $W_{r}^{\prime}$ est $\mathcal{A}$-mesurable, on a $\lim _{t \longrightarrow 0} \lambda^{*}\left(W_{t}\right) \leq \lim _{r \longrightarrow 0} \lambda\left(W_{r}^{\prime}\right)=0$.

On montre d'abord que $E$ coupe toute boule ouverte centrée dans $F$; en effet soit $x \in F$ et soit $r>0$; soit $\beta=\theta(r) \wedge \eta(x, r)$; soit $x^{\prime} \in D$ avec $d\left(x, x^{\prime}\right)<\beta$; pour tout $z \in \mathbf{U}_{d}\left(x^{\prime}, \beta\right)$, on a $d\left(x^{\prime}, z\right)<\eta(x, r)$; comme on a $d\left(x, x^{\prime}\right)<\theta(r)$, on trouve $d(x, z)<r ;$ donc $\mathbf{U}_{d}(x, r)$, qui contient $\mathbf{U}_{d}\left(x^{\prime}, \beta\right)$ (qui coupe $F$ ), contient un point de $E$.

Il reste à montrer que $W_{t} \subset W_{r}^{\prime}$ pour $\left.\left.t \in\right] 0, \theta(r)\right]$. Soit $y$ un point de $W_{t}$; il existe $x \in F$ avec $d(y, x)<t \leq \theta(r)$; on choisit $x^{\prime} \in E$ avec $d\left(x, x^{\prime}\right)<\eta(y, r)$, donc $d\left(y, x^{\prime}\right)<r$;

\section{(3.3) Dérivation en mesure}

Lorsque $(X, d)$ possède la $\operatorname{prf}(m)$ restreinte aux boules à rayon constant, on peut montrer que les quotients $T_{r}(\nu, \lambda)$ convergent en mesure dès qu'on a des hypothèses de mesurabilité convenables (3.2.2a ou bien $3.2 .2 \mathrm{~b}$ ), par exemple dès que $d$ est un écart et que $\mathcal{A} \supset \mathcal{B}_{d}$; on montre même que la convergence a lieu dans $L^{1}$ si $\nu=f \lambda$. 
Proposition 3.3.1. Soit $X$ un ensemble muni d'un noyau symétrique d avec $\mathcal{A}_{d} \subset \mathcal{B}_{d}$, et soit $Y \subset X ;$ soit $\mathcal{A}$ une tribu sur $X$ avec $\mathcal{A} \supset \mathcal{B}_{d}$. On suppose que :

(i) $(X, d)$ vérifie $3.2 .2 \mathrm{a}$ (par exemple d est un écart) ou bien $3.2 .2 \mathrm{~b}$;

(ii) $(X, d)$ possède la $\operatorname{prf}(m)$ restreinte aux boules centrées dans $Y$ et à rayon constant (pour un $m$ entier $\geq 1$ ).

On a alors :

(a) pour tout $\lambda \in \mathcal{P}(X, \mathcal{A})$ et tout $f \in L^{1}\left(X, \mathcal{B}_{d}, \lambda\right)$, le quotient de dérivation $T_{r}(f \lambda, \lambda)$ tend vers $f \mathbf{I}_{X \backslash Z(\lambda, d)}$ dans $L^{1}(Y, \mathcal{A}, \lambda)$ lorsque $r$ tend vers 0 .

Si $\lambda^{*}(Z(\lambda, d))=0$ (ce qui est le cas "normal", voir 2.6), on en déduit les conséquences suivantes:

(b) pour tout $\lambda \in \mathcal{P}(X, \mathcal{A})$ et tout $f \in L^{1}\left(X, \mathcal{B}_{d}, \lambda\right)$, le quotient de dérivation $T_{r}(f \lambda, \lambda)$ tend vers $f$ dans $L^{1}(Y, \mathcal{A}, \lambda)$ lorsque $r$ tend vers 0.

(c) pour tout $\lambda \in \mathcal{P}(X, \mathcal{A})$ et tout $f \in L^{1}(X, \mathcal{A}, \lambda)$, le quotient de dérivation $T_{r}(f \lambda, \lambda)$ tend vers $\mathbb{E}_{\lambda}\left(f \mid \mathcal{B}_{d}\right)$ dans $L^{1}(Y, \mathcal{A}, \lambda)$ lorsque $r$ tend vers 0 .

\section{Démonstration.}

Démonstration de (a). Par 2.4c, on choisit une fonction $g \mathcal{A}$-mesurable continue bornée $\operatorname{sur}\left(X, \mathcal{T}_{d}\right)$ approchant $f$ à $\varepsilon$ près dans $L^{1}(X, \mathcal{A}, \lambda)$; soit $k=f-g$.

En appliquant 3.2.3, on voit que

$$
\left.\lim _{r \longrightarrow 0} \lambda^{*}(X \backslash Z(\lambda, d)) \cap\left\{\left|T_{r}(g \lambda, \lambda)-g\right|>\alpha\right\}\right)=0
$$

donc que $T_{r}(g \lambda, \lambda)$ converge en mesure vers $g \mathbf{I}_{X \backslash Z(\lambda, d)}$ lorsque $r$ tend vers 0 .

Par convergence dominée, cette convergence a même lieu dans $L^{1}(X, \mathcal{A}, \lambda)$ (voir 3.2.1c). De plus, par 3.1, on a :

$$
\mathbb{E}_{\lambda}^{*}\left(\left|T_{r}(k \lambda, \lambda)-k \mathbf{I}_{X \backslash Z(\lambda, d)}\right| \mathbf{I}_{Y}\right) \leq \mathbb{E}_{\lambda}^{*}\left(T_{r}(|k| \lambda, \lambda) \mathbf{I}_{Y}\right)+\mathbb{E}_{\lambda}(|k|) \leq m \varepsilon+\varepsilon .
$$

Comme $\varepsilon$ est arbitraire, $T_{r}(f \lambda, \lambda)$ converge vers $f \mathbf{I}_{X \backslash Z(\lambda, d)}$ dans $L^{1}(Y, \mathcal{A}, \lambda)$.

Démonstration de (b). Ce point est évident.

Démonstration de (c). Soit $f \in L^{1}(X, \mathcal{A}, \lambda)$, on pose $f_{1}=\mathbb{E}_{\lambda}\left(f \mid \mathcal{B}_{d}\right)$ et $\nu_{1}=f_{1} \lambda$. On a $\nu\left(\mathbf{B}_{d}(y, r)\right)=\nu_{1}\left(\mathbf{B}_{d}(y, r)\right)$ pour tout $y$ et tout $r$. Donc $T_{r}(f \lambda, \lambda)=T_{r}\left(f_{1} \lambda, \lambda\right)$ tend vers $f_{1}$ dans $L^{1}(Y, \mathcal{A}, \lambda)$ si $r$ tend vers 0 .

Il reste à traiter le cas où $\nu$ est étrangère à $\lambda$. 
Proposition 3.3.2. $\quad$ Soit $X$ un ensemble muni d'un noyau symétrique d avec $\mathcal{A}_{d} \subset \mathcal{B}_{d}$, et soit $Y \subset X ;$ soit $\mathcal{A}$ une tribu sur $X$ avec $\mathcal{A} \supset \mathcal{B}_{d}$. On suppose que :

(i) $(X, d)$ vérifie 3.2.2a (par exemple d est un écart et $\mathcal{A} \supset \mathcal{B}_{d}$ ) ou bien $3.2 .2 \mathrm{~b}$;

(ii) $(X, d)$ possède la $\operatorname{prf}(m)$ restreinte aux boules centrées dans $Y$ et à rayon constant (pour un $m$ entier $\geq 1$ ).

On a alors :

(a) pour tout $\lambda \in \mathcal{P}(X, \mathcal{A})$, tout $\nu \in \mathcal{M}_{1}^{+}(X, \mathcal{A})$, tout $B \in \mathcal{B}_{d}$ et tout $\alpha>0$, on $a$ :

$$
\limsup _{r \longrightarrow 0} \alpha \lambda^{*}\left(Y \cap B \cap\left\{T_{r}(\nu, \lambda)>\alpha\right\}\right) \leq m \nu(B) ;
$$

(b) soient $\lambda \in \mathcal{P}\left(X, \mathcal{B}_{d}\right)$ et $\nu \in \mathcal{M}_{1}^{+}\left(X, \mathcal{B}_{d}\right)$ étrangère à $\lambda$; alors $T_{r}(\nu, \lambda)$ converge vers 0 en mesure (relativement à $\lambda$ ) sur $Y$ lorsque $r$ tend vers 0 .

Démonstration. Démonstration de (a). Soient $\lambda \in \mathcal{P}(X, \mathcal{A}), \nu \in$ $\mathcal{M}_{1}^{+}(X, \mathcal{A}), B \in \mathcal{B}_{d}$ et $\alpha>0$. Soit $\varepsilon>0$. Par 2.4a, on choisit $V \in \mathcal{B}_{d}$ ouvert avec $B \subset V$ et $\nu(V \backslash B)<\varepsilon$; soit $F=X \backslash V$. On note $\lambda_{V}$ et $\nu_{V}$ les traces de $\lambda$ et $\nu$ sur $V$.

Pour tout $t>0$, on pose $W_{t}=\{y \in X \mid 0<d(y, F)<t\}$; on a donc $B \backslash W_{t}=\left\{y \in B \mid \mathbf{U}_{d}(y, t) \subset V\right\}$. Donc, pour $y \in B \backslash W_{t}$, on a $\mathbf{U}_{d}(y, t) \subset V$, donc $T_{r}(\nu, \lambda)(y)=T_{r}\left(\nu_{V}, \lambda_{V}\right)(y)$ pour tout $r<t$. Grâce à 3.2.4, on peut choisir $t>0$ tel que $\lambda^{*}\left(W_{t}\right) \leq \varepsilon$.

On applique l'inégalité 3.1 (g3) aux mesures $\lambda_{V}$ et $\nu_{V}$ :

$$
\begin{aligned}
\alpha \lambda^{*}\left(Y \cap V \cap\left\{T_{r}\left(\nu_{V}, \lambda_{V}\right)>\alpha\right\}\right) & \leq \mathbb{E}_{\lambda}^{*}\left(T_{r}\left(\nu_{V}, \lambda_{V}\right) \mathbf{I}_{Y \cap V}\right) \\
& \leq m \nu_{V}(X)=m \nu(V) \leq m \nu(B)+m \varepsilon
\end{aligned}
$$

on a donc, pour tout $r<t$ :

$$
\begin{aligned}
& \alpha \lambda^{*}\left(Y \cap B \cap\left\{T_{r}(\nu, \lambda)>\alpha\right\}\right) \\
& \quad \leq \alpha \lambda^{*}\left(Y \cap\left(B \backslash W_{t}\right) \cap\left\{T_{r}(\nu, \lambda)>\alpha\right\}\right)+\alpha \lambda^{*}\left(W_{t}\right) \leq m \nu(B)+m \varepsilon+\alpha \varepsilon .
\end{aligned}
$$

On a donc $\lim \sup _{r \longrightarrow 0} \alpha \lambda^{*}\left(Y \cap B \cap\left\{T_{r}(\nu, \lambda)>\alpha\right\}\right) \leq m \nu(B)+m \varepsilon+\alpha \varepsilon$.

En faisant tendre $\varepsilon$ vers 0 , on obtient :

$$
\limsup _{r \longrightarrow 0} \alpha \lambda^{*}\left(Y \cap B \cap\left\{T_{r}(\nu, \lambda)>\alpha\right\}\right) \leq m \nu(B) .
$$

Démonstration de (b). Soit $B \in \mathcal{B}_{d}$ avec $\lambda(B)=1$ et $\nu(B)=0$. Appliquant (a) à $B$, on trouve que l'on a :

$$
\limsup _{r \longrightarrow 0} \alpha \lambda^{*}\left(Y \cap B \cap\left\{T_{r}(\nu, \lambda)>\alpha\right\}\right)=0 \quad \text { pour tout } \alpha>0 .
$$


Dans le cas de deux mesures étrangères $\lambda$ et $\nu$, la convergence de $T_{r}(\nu, \lambda)$ n'a pas lieu en général dans $L^{1}$ :

en effet, supposons que $d$ soit l'ultramétrique associée à la famille croissante des tribus dyadiques sur [0,1[ (voir 4.7c); on se donne deux lois de probabilités $\lambda$ et $\nu$ étrangères et chargeant tout intervalle ouvert non vide ; alors on a $\mathbb{E}_{\lambda}\left(T_{r}(\nu, \lambda)\right)=1$ pour tout $r>0$; donc $T_{r}(\nu, \lambda)$ ne peut tendre vers 0 dans $L^{1}$.

(3.3.3) On a $\mathcal{A}_{d} \subset \mathcal{B}_{d}$ dès qu'on peut choisir une topologie $\mathcal{T}$ sur $X$ telle que :

(a) toute boule de $(X, d)$ est $\mathcal{B}(\mathcal{T})$-mesurable (i.e. $\mathcal{A}_{d} \subset \mathcal{B}(\mathcal{T})$ ),

(b) pour tout $y \in X$ et tout voisinage $V$ de $y$ dans $(X, \mathcal{T})$, il existe $r>0$ avec $\mathbf{B}_{d}(y, r) \subset V$ (i.e. $\mathcal{T} \subset \mathcal{T}_{d}$, donc $\left.\mathcal{B}(\mathcal{T}) \subset \mathcal{B}_{d}\right)$.

La réciproque, qui n'a pas d'intérêt, est vraie (prendre $\mathcal{T}=\mathcal{T}_{d}$ ).

On verra en $4.7 \mathrm{e}$ que cette remarque présente tout de même un certain intérêt lorsqu'on considère plusieurs noyaux $d_{i}$ vérifiant $(\mathrm{a})$ et $(\mathrm{b})$ pour une même topologie $\mathcal{T}$.

\section{(3.4) Equi-intégrabilité}

Soit $X$ un ensemble muni d'un noyau symétrique quelconque $d$ avec $\mathcal{A}_{d} \subset$ $\mathcal{B}_{d} ;$ soit $\mathcal{A}$ une tribu sur $X$ avec $\mathcal{A} \supset \mathcal{A}_{d}$. On suppose que $(X, d)$ possède la propriété de recouvrement faible de degré $\leq m$ restreinte aux familles de boules fermées à rayon constant (pour un $m$ entier $\geq 1$ ).

Soient $\lambda \in \mathcal{P}(X, \mathcal{A})$ et $f \in L^{1}(X, \mathcal{A}, \lambda)$.

(a) Pour toute fonction $S$, on note $S^{*}$ la fonction $\mathcal{A}$-mesurable (unique $\lambda$-presque sûrement) vérifiant $S^{*} \geq S$ et $\mathbb{E}_{\lambda}\left(S^{*}\right)=\mathbb{E}_{\lambda}^{*}(S)$; alors on a $\varphi\left(T_{r}(|f| \lambda, \lambda)^{*}\right)=\varphi\left(T_{r}(|f| \lambda, \lambda)\right)^{*}$ pour toute $\varphi$ continue et croissante (voir $[40, \mathrm{p} .13])$.

(b) On a $\varphi\left(T_{r}(|f| \lambda, \lambda)\right) \leq T_{r}((\varphi \circ|f|) \lambda, \lambda)$ pour toute $\varphi$ convexe et croissante ; on a donc par 3.1 :

$$
\mathbb{E}_{\lambda}^{*}\left(\varphi\left(T_{r}(|f| \lambda, \lambda)\right)\right) \leq \mathbb{E}_{\lambda}^{*}\left(T_{r}((\varphi \circ|f|) \lambda, \lambda)\right) \leq m \mathbb{E}_{\lambda}(\varphi \circ|f|) .
$$

On a donc $\mathbb{E}_{\lambda}\left(\varphi\left(T_{r}(|f| \lambda, \lambda)^{*}\right)\right) \leq m \mathbb{E}_{\lambda}(\varphi \circ|f|)$ pour tout $r$.

(c) On choisit $\varphi$ convexe et croissante de façon que $\mathbb{E}_{\lambda}(\varphi \circ|f|)$ soit fini et que $\varphi(t) / t$ tende vers $+\infty$ lorsque $t$ tend vers $+\infty$.

En utilisant le critère de La Vallée-Poussin (voir [27, p. 38]), on voit que les fonctions $\left(T_{r}(f \lambda, \lambda)^{*}\right)_{0<r<+\infty}$ sont $\lambda$-équi-intégrables; rappelons que cela tient à ce qu'on a :

$$
\mathbb{E}_{\lambda}\left(T_{r}^{*} \mathbf{I}_{\left\{T_{r}^{*}>\alpha\right\}}\right) \leq[\alpha / \varphi(\alpha)] \mathbb{E}_{\lambda}\left(\varphi\left(T_{r}^{*}\right)\right) \leq \varepsilon \text { pour } \alpha \text { assez grand } .
$$


(d) Dans la proposition 3.3.1, la convergence des $T_{r}(f \lambda, \lambda)$ vers $\mathbb{E}_{\lambda}\left(f \mid \mathcal{B}_{d}\right)$ a donc lieu aussi dans $L^{p}(Y, \mathcal{A}, \lambda), p>1$, dès que $f \in L^{p}(X, \mathcal{A}, \lambda)$.

\section{(3.5) Dimension de De Groot}

Soit $X$ un ensemble muni d'un noyau symétrique $d$.

(a) L'espace $(X, d)$ est dit de dimension de De Groot $\leq n$ si :

(g0) pour tout $a \in X$ et tous $y_{i} \in X(i=0, \ldots, n+1)$, il existe $i, j$ distincts et $\ell$ avec $d\left(y_{i}, y_{j}\right) \leq d\left(a, y_{\ell}\right)$.

(b) Soit $n$ un entier $\geq 0$; on voit aisément que $(X, d)$ est de dimension de De Groot $\leq n$ si et seulement si il possède pour tout $r>0$ la propriété suivante (propriété (g1) dans 3.1) :

pour tous $a \in X$ et tous $y_{0}, \ldots, y_{n+1} \in \mathbf{B}_{d}(a, r)$, il existe $i, j$ distincts avec $d\left(y_{i}, y_{j}\right) \leq r$

En effet, si on a (g1) pour tout $r>0$, on en déduit $(\mathrm{g} 0)$ en prenant $r=d\left(a, y_{\ell}\right)=\sup _{i \in I} d\left(a, y_{i}\right) ;$ l'implication inverse est évidente.

Par 3.1, on voit donc que $(X, d)$ est de dimension de De Groot $\leq n$ si et seulement il possède la propriété de recouvrement faible de degré $\leq n+1$ restreinte aux familles de boules fermées à rayon constant.

(c) Le cas $n=0$ correspond aux ultramétriques (i.e. les noyaux symétriques $d$ vérifiant $d(x, y) \leq d(x, z) \vee d(z, y)$ pour tous $x, y, z \in X)$.

En fait une ultramétrique est même de dimension de Nagata 0 (voir 4.7c).

(d) Pour un espace métrique de dimension métrique finie (voir 6.1.1), la dimension de De Groot est toujours finie, puisqu'elle vaut $\kappa(1)-1$, où $\kappa(1)$ est le plus petit entier $\kappa$ tel que, pour tout $y \in X$ et tout $r>0$, on puisse trouver au plus $\kappa$ points de $\mathbf{B}_{d}(y, r)$ de distances mutuelles $>r$ pour $d$ (voir plus loin en 5.1).

La dimension de De Groot intervient dans un résultat de nature topologique :

Théorème de De Groot ([17]). Un espace métrisable précompact X est de dimension topologique $\leq n$ dès que sa topologie peut être définie par une distance d telle que $(X, d)$ soit de dimension de De Groot $\leq n$.

L'inverse est vrai sans qu'il soit besoin de supposer $X$ précompact (ce qui résulte en fait du théorème de Nagata-Ostrand, voir 4.8).

\section{(3.6) Conditions portant seulement sur $\lambda$}

Soit $X$ un ensemble muni d'un noyau symétrique $d$ avec $\mathcal{A}_{d} \subset \mathcal{B}_{d}$; soit $\mathcal{A}$ une tribu sur $X$ avec $\mathcal{A} \supset \mathcal{A}_{d}$. Soit $m \in \mathbb{N}$.

On fixe $\lambda \in \mathcal{P}(X, \mathcal{A})$ et on se propose de voir sous quelles conditions on a convergence en mesure de $T_{r}(\nu, \lambda)$ pour tout $\nu \in \mathcal{M}_{1}^{+}(X, \mathcal{A})$. 
Pour simplifier, on suppose que $T_{r}(\nu, \lambda)$ est $\mathcal{A}$-mesurable et $Z(\lambda, d)$ est $\lambda$-négligeable pour tous $\lambda, \nu$ (c'est le cas si $(X, d)$ est un espace métrique séparable) ; en particulier, sauf sur un ensemble $\lambda$-négligeable, on ne se trouve jamais dans le cas $\lambda\left(\mathbf{B}_{d}(y, r)\right)=\nu\left(\mathbf{B}_{d}(y, r)\right)=0$.

On considère les propriétés suivantes :

(a) $\lambda\left(\mathbf{B}_{d}(a, r)\right) \leq m \lambda\left(\mathbf{B}_{d}(y, r)\right)$ pour $\lambda$-presque tout $y \in \mathbf{B}_{d}(a, r)$,

(b) $\mathbb{E}_{\lambda}\left(T_{r}\left(\delta_{a}, \lambda\right)\right) \leq m$ pour tout $a \in X$ et tout $r>0$,

(c) $\mathbb{E}_{\lambda}\left(T_{r}(\nu, \lambda)\right) \leq m \nu(X)$ pour tout $\nu \in \mathcal{M}_{1}^{+}(X, \mathcal{A})$,

(d) $T_{r}(\nu, \lambda)$ converge en mesure pour tout $\nu \in \mathcal{M}_{1}^{+}(X, \mathcal{A})$.

On a alors $(\mathrm{a}) \Longrightarrow(\mathrm{b}) \Longrightarrow(\mathrm{c}) \Longrightarrow(\mathrm{d})$.

Pour obtenir $(\mathrm{a}) \Longrightarrow(\mathrm{b})$, on note qu'on a $T_{r}\left(\delta_{a}, \lambda\right)(y)=0$ si $y \notin \mathbf{B}_{d}(a, r)$, et que $T_{r}\left(\delta_{a}, \lambda\right)(y)=1 / \lambda\left(\mathbf{B}_{d}(y, r)\right) \leq m / \lambda\left(\mathbf{B}_{d}(a, r)\right)$ pour $\lambda$-presque tout $y \in \mathbf{B}_{d}(a, r)$.

Pour obtenir $(\mathrm{b}) \Longrightarrow(\mathrm{c})$, on note qu'on a par Fubini $\mathbb{E}_{\lambda}\left(T_{r}(\nu, \lambda)\right)=\int \mathbb{E}_{\lambda}\left(T_{r}\left(\delta_{x}, \lambda\right)\right) \nu(d x) \leq m \nu(X)$ pour tout $\nu \in \mathcal{M}_{1}^{+}(X, \mathcal{A})$.

Enfin on a $(\mathrm{c}) \Longrightarrow(\mathrm{d})$; en effet, un examen des démontrations de 3.3.1 et 3.3.2 montre que les propriétés de recouvrement n'y interviennent que par l'inégalité (c).

\section{(3.7) Une autre démonstration de la convergence en mesure}

(3.7.1) Soit $X$ un ensemble muni d'un noyau symétrique $d$ avec $\mathcal{A}_{d} \subset \mathcal{B}_{d}$. Soit $\mathcal{A}$ une tribu sur $X$ avec $\mathcal{A} \supset \mathcal{B}_{d}$ et soit $\lambda \in \mathcal{P}(X, \mathcal{A})$. Soit $f$ une fonction $\mathcal{B}_{d}$-mesurable, partout finie et $\lambda$-intégrable de $X$ dans $\mathbb{R}$.

On a vu en 2.4.3 que :

(a) pour tout $\varepsilon>0$, il existe une jauge $\gamma$ sur $X$ et une mesure $\mu \epsilon$ $\mathcal{M}_{1}^{+}\left(X, \mathcal{B}_{d}\right)(\operatorname{avec} \mu(X) \leq 1)$ tels que :

$$
\left|f \lambda\left(\mathbf{B}_{d}(y, r)\right)-f(y) \lambda\left(\mathbf{B}_{d}(y, r)\right)\right| \leq \varepsilon \mu\left(\mathbf{B}_{d}(y, r)\right)
$$

pour tous $y \in X, r \in] 0, \gamma(y)[$.

(3.7.2) Si $(X, d)$ vérifie la propriété de recouvrement faible restreinte aux familles de boules à rayon constant centrées dans $Y$, et si on est à même d'affirmer que, dans (a), $\gamma$ peut être choisi $\lambda$-mesurable, on peut montrer que $T_{r}(f \lambda, \lambda)$ tend vers $f \mathbf{I}_{X \backslash Z(\lambda, d)}$ en mesure dans $Y$ (relativement à $\lambda$ ) lorsque $r$ tend vers 0 . 
Démonstration. On pose $Z=Z(\lambda, d)$. Soient $\alpha>0$ et $r>0$; on pose :

$$
Y_{\alpha, r}=Y \cap\left\{\left|T_{r}(f \lambda, \lambda)-f \mathbf{I}_{X \backslash Z}\right|>\alpha\right\} .
$$

Soit $\varepsilon>0$; on se donne $\gamma$ et $\mu$ associés à $\varepsilon$ par (a) et on suppose que $\gamma$ est $\lambda$-mesurable. On pose $U=\left\{y \in Y \mid \gamma(y)>r_{0}\right\}$ où $r_{0}$ est choisi assez petit pour que $\lambda(X \backslash U)<\varepsilon$.

Soit $r \leq r_{0}$. On pose $B(y)=\mathbf{B}_{d}(y, r)$ pour chaque $y \in U \cap Y_{\alpha, r}$. On a donc :

$$
\left|T_{r}(f \lambda, \lambda)(y)-f(y) \mathbf{I}_{X \backslash Z}(y)\right|>\alpha(\text { donc } y \notin Z) .
$$

On a donc, en utilisant (a) :

$$
\alpha \lambda(B(y))<|f \lambda(B(y))-f(y) \lambda(B(y))| \leq \varepsilon \mu(B(y)) .
$$

On peut extraire de $U \cap Y_{\alpha, r}$ une famille de points $\left(y_{i}\right)_{i \in I}$ telle que la famille $\left(B\left(y_{i}\right)\right)_{i \in I}$ recouvre $U \cap Y_{\alpha, r}$ et soit d'ordre $\leq m$; or $\lambda$ charge chaque $B\left(y_{i}\right)$ pour $i \in I$; donc $I$ est dénombrable, par 2.5.1. On a alors :

$$
\alpha \lambda^{*}\left(U \cap Y_{\alpha, r}\right) \leq \sum_{i} \alpha \lambda\left(B\left(y_{i}\right)\right) \leq \sum_{i \in I} \varepsilon \mu\left(B\left(y_{i}\right)\right) \leq m \varepsilon \mu(X) .
$$

Donc $\lambda^{*}\left(Y_{\alpha, r}\right) \leq \lambda^{*}\left(U \cap Y_{\alpha, r}\right)+\lambda(X \backslash U) \leq(m+1) \varepsilon$ pour tout $r \leq r_{0}$. Donc, pour tout $\alpha>0, \lambda^{*}\left(Y_{\alpha, r}\right)$ tend vers 0 lorsque $r$ tend vers 0 .

(3.7.3) Si $(X, d)$ vérifie la propriété de recouvrement faible restreinte aux familles de boules à rayon constant centrées dans $Y$, et que $\gamma$ ne peut pas être choisi $\lambda$-mesurable, on peut cependant montrer que :

quelle que soit la suite $\left(r_{n}\right)_{n \in \mathbb{N}}$ tendant vers $0\left(r_{n}>0\right), T_{r_{n}}(f \lambda, \lambda)$ tend vers $f \mathbf{I}_{X \backslash Z(\lambda, d)}$ en mesure dans $Y$ (relativement à $\lambda$ ) lorsque $n$ tend vers $+\infty$.

\section{Dérivation des mesures (convergence presque sûre)}

L'objectif de ce paragraphe est d'arriver à la dérivation presque sûre des mesures par les étapes mentionnées dans l'introduction. Les résultats principaux se trouvent en 4.3 (inégalité maximale) et en 4.4 (dérivation).

Les propriétés utilisées dans les énoncés 4.2 à 4.4 sont numérotées dans leur ensemble de $(\mathrm{n} 1)$ à $(\mathrm{n} 7)$; les propriétés (n1) [dimension de Nagata $\leq m-1],(\mathrm{n} 2),(\mathrm{n} 3)$ [propriété de recouvrement faible de degré $\leq m$ ] et (n4) [inégalité maximale] sont toutes équivalentes. Si on veut arriver plus rapidement au théorème de dérivation, on peut ignorer les réciproques et se contenter des implications

$$
(n 1) \Longrightarrow(n 2) \Longrightarrow(n 3) \Longrightarrow(n 4) \Longrightarrow(n 5) \Longrightarrow(n 6) \Longrightarrow(n 7) .
$$


La relation entre la propriété de recouvrement faible et la dimension de Nagata (liée à la dimension topologique) est explicitée en 4.7.

On montre enfin en 4.9 que le degré exact de la propriété de recouvrement faible dans un espace à courbure $\geq 0$ est en fait le cardinal d'un réseau ; c'est notamment le cas de $\mathbb{R}^{n}$ muni de la distance associée à une norme.

$\mathrm{Au}$ lieu de supposer que $(X, d)$ vérifie la propriété de recouvrement faible de degré $\leq m$, on supposera généralement dans ce paragraphe que $(X, d)$ vérifie seulement la propriété de recouvrement faible de degré $\leq m$ restreinte aux familles de boules fermées centrées dans $Y$ (en bref $\operatorname{prf}(m)$ pour les boules centrées dans $Y$ ), où $Y$ est une partie de $X$.

(4.1) On dit qu'une famille $\mathcal{B}=\left\{\mathbf{B}_{d}\left(y_{i}, r_{i}\right)\right\}_{i \in I}$ est sans liaison si on a $y_{i} \notin \mathbf{B}_{d}\left(y_{j}, r_{j}\right)$ pour tous $i, j$ distincts dans $I$.

Lemme 4.2. Soit $X$ un ensemble muni d'un noyau symétrique quelconque $d$ et soit $Y \subset X$. Alors les assertions suivantes sont équivalentes:

(n1) pour tous $a \in X$ et $y_{1}, \ldots, y_{m+1} \in Y$, il existe $i, j$ distincts avec

$$
d\left(y_{i}, y_{j}\right) \leq d\left(a, y_{i}\right) \vee d\left(a, y_{j}\right)
$$

(n2) toute famille sans liaison de boules fermées centrées dans $Y$ est d'ordre $\leq m$ dans $X$

(n3) $(X, d)$ possède la $\operatorname{prf}(m)$ restreinte aux boules centrées dans $Y$.

\section{Démonstration.}

Démonstration de $(n 1) \Longrightarrow(n 2)$. Soient $\mathbf{B}_{d}\left(y_{1}, r_{1}\right), \ldots, \mathbf{B}_{d}\left(y_{k}, r_{k}\right)$ une famille sans liaison de boules fermées centrées dans $Y$ contenant un point $a$ de $X$; on a alors $d\left(y_{i}, y_{j}\right)>r_{i} \vee r_{j} \geq d\left(a, y_{i}\right) \vee d\left(a, y_{j}\right)$ pour tous $i, j$ distincts, donc (n1) implique $k \leq m$.

Démonstration de (n2) $\Longrightarrow(n 3)$. Soit $\left\{\mathbf{B}_{d}\left(y_{i}, r_{i}\right)\right\}_{i \in I}$ des boules centrées dans $Y$ telles que les rayons $\left(r_{i}\right)_{i \in I}$ soient dans une suite décroissante $\left(v_{k}\right)_{k \in \mathbb{N}}$; on pose $B_{i}=\mathbf{B}_{d}\left(y_{i}, r_{i}\right)$ pour alléger les notations.

Soit $J_{1}$ une partie maximale de $\left\{i \in I \mid r_{i}=v_{1}\right\}$ telle que $d\left(y_{i}, y_{j}\right)>v_{1}$ pour tous $i, j$ distincts dans $J_{1}$. Supposons $J_{1}, \ldots, J_{k-1}$ définis ; notons $X_{k-1}$ la réunion des boules $B_{j}$ pour tout $j \in J_{1} \cup \ldots \cup J_{k-1}$; soit $J_{k}$ une partie maximale de $\left\{i \in I \mid r_{i}=v_{k}, y_{i} \notin X_{k-1}\right\}$ telle que $d\left(y_{i}, y_{j}\right)>v_{k}$ pour tous $i, j$ distincts dans $J_{k}$. La réunion $J$ des $J_{k}$ vérifie alors :

- la réunion des $B_{j}(j \in J)$ contient $y_{i}$ pour tout $i \in I$,

- on a $y_{i} \notin B_{j}$ pour tous $i, j$ distincts dans $J$, donc (n2) implique que la famille $\left\{\mathbf{B}_{d}\left(y_{j}, r_{j}\right)\right\}_{j \in J}$ est d'ordre $\leq m$ dans $X$. 
Démonstration de (n3) $\Longrightarrow(n 1)$. Soient $a \in X$ et $y_{1}, \ldots, y_{k} \in Y$ vérifiant $d\left(y_{i}, y_{j}\right)>d\left(a, y_{i}\right) \vee d\left(a, y_{j}\right)$ pour tous $i, j$ distincts ; les boules $B_{i}=\mathbf{B}_{d}\left(y_{i}, d\left(a, y_{i}\right)\right)$ vérifient alors $y_{i} \notin B_{j}$ pour tous $i, j$ distincts ; de plus chacune d'elles contient $a$; or aucune sous-famille propre ne contient tous les $y_{j}$, donc (n3) implique $k \leq m$.

Lemme 4.3. Soit $X$ un ensemble muni d'un noyau symétrique quelconque $d$ et soit $Y \subset X$; soit $\mathcal{A}$ une tribu sur $X$ avec $\mathcal{A} \supset \mathcal{A}_{d}$. Alors les assertions suivantes sont équivalentes :

(n3) $(X, d)$ possède la $\operatorname{prf}(m)$ restreinte aux boules centrées dans $Y$.

(n4) (inégalité maximale)

Pour tout $\lambda \in \mathcal{P}(X, \mathcal{A})$, tout $\nu \in \mathcal{M}_{1}^{+}(X, \mathcal{A})$, tout $\left.r \in\right] 0,+\infty[$ et tout $\alpha>0$, on $a \lambda^{*}\left(Y \cap\left\{S_{r}(\nu, \lambda)>\alpha\right\}\right) \leq m|\nu|(X)$

(on rappelle qu'on pose $S_{r}(\nu, \lambda)=\sup _{0<u<r} T_{u}(\nu, \lambda)$ ).

\section{Démonstration.}

Démonstration de (n3) $\Longrightarrow\left(n_{4}\right)$. Soient $\lambda \in \mathcal{P}(X, \mathcal{A}), \nu \in \mathcal{M}_{1}^{+}(X, \mathcal{A})$ et $\alpha>0$. Soit $Y_{\alpha}^{r}=\left\{y \in Y \mid S_{r}(\nu, \lambda)(y)>\alpha\right\}$; pour chaque $y \in Y_{\alpha}^{r}$, on choisit $r_{y}$ avec $\alpha \lambda\left(\mathbf{B}_{d}\left(y, r_{y}\right)\right)<\nu\left(\mathbf{B}_{d}\left(y, r_{y}\right)\right)$; quitte à l'augmenter un peu, on peut supposer que $r_{y}$ est rationnel. On pose $B(y)=\mathbf{B}_{d}\left(y, r_{y}\right)$ pour chaque $y \in Y_{\alpha}^{r}$. On considère $\mathbb{Q}$ comme une réunion croissante d'ensembles finis $D_{j}$ et on note $Y_{\alpha, j}$ l'ensemble des $y \in Y_{\alpha}^{r}$ avec $r_{y} \in D_{j}$.

Fixons d'abord $j$; on peut extraire de $Y_{\alpha, j}$ une famille de points $\left(y_{i}\right)_{i \in I}$ telle que la famille $\left(B\left(y_{i}\right)\right)_{i \in I}$ recouvre $Y_{\alpha, j}$ et soit d'ordre $\leq m$; or la mesure $\nu$ charge chaque $B\left(y_{i}\right)$ pour $i \in I$; donc $I$ est dénombrable, par 2.5.1. On a donc :

$$
\begin{aligned}
\alpha \lambda^{*}\left(Y_{\alpha, j}\right) & \leq \alpha \sum_{i \in I} \lambda\left(B\left(y_{i}\right)\right)<\sum_{i \in I} \nu\left(B\left(y_{i}\right)\right) \\
& \leq m|\nu|\left(\cup_{i \in I} B\left(y_{i}\right)\right) \leq m|\nu|(X) .
\end{aligned}
$$

En faisant varier $j$, on en déduit qu'on a $\alpha \lambda^{*}\left(Y_{\alpha}^{r}\right) \leq m|\nu|(X)$.

Démonstration de $\left(n_{4}\right) \Longrightarrow(n 3)$. Soient $\mathbf{B}_{d}\left(y_{1}, r_{1}\right), \ldots, \mathbf{B}_{d}\left(y_{k}, r_{k}\right)$ des boules centrées dans $Y$ vérifiant $y_{i} \notin \mathbf{B}_{d}\left(y_{j}, r_{j}\right)$ pour tous $i, j$ distincts et contenant un point $a$ de $X$; soit $r>\sup _{i} r_{i}\left(r\right.$ fini). On pose $\mathbf{B}_{d}\left(y_{i}, r_{i}\right)=$ $B_{i}$; soit $\lambda=\frac{1}{k}\left(\delta_{y_{1}}+\ldots+\delta_{y_{k}}\right)$ et soit $\nu=\delta_{a}$. En appliquant (n4) avec $\alpha=m+\frac{1}{2}$, on obtient $\lambda\left(\left\{S_{r}(\nu, \lambda)>m+\frac{1}{2}\right\}\right)<1$; il existe donc $i$ tel que $S_{r}(\nu, \lambda)\left(y_{i}\right) \leq m+\frac{1}{2}$; comme on a $r_{i}<r$, on a $\nu\left(B_{i}\right) / \lambda\left(B_{i}\right) \leq m+\frac{1}{2}$; or $\nu\left(B_{i}\right)=1\left(\operatorname{car} a \in B_{i}\right)$ et $\lambda\left(B_{i}\right)=1 / k\left(\operatorname{car} y_{j} \notin B_{i}\right.$ pour tout $\left.j \neq i\right)$; on a donc $k \leq m+\frac{1}{2}$, donc $k \leq m$. On a donc montré (n2), dont on déduit (n3) par 4.2 . 
Le passage de l'inégalité maximale au théorème de dérivation est classique dans des cas particuliers (voir par exemple [36], [18], [42]) :

Théorème 4.4. Soit $X$ un ensemble muni d'un noyau symétrique quelconque $d$ avec $\mathcal{A}_{d} \subset \mathcal{B}_{d}$, et soit $Y \subset X$. Soit $\mathcal{A}$ une tribu sur $X$ avec $\mathcal{A} \supset \mathcal{B}_{d}$. Supposons que $(X, d)$ vérifie l'inégalité maximale :

(n4) pour tout $\lambda \in \mathcal{P}(X, \mathcal{A})$, tout $\nu \in \mathcal{M}_{1}(X, \mathcal{A})$, tout $\left.r \in\right] 0,+\infty[$ et tout $\alpha>0$, on $a \alpha \lambda^{*}\left(Y \cap\left\{S_{r}(\nu, \lambda)>\alpha\right\}\right) \leq m|\nu|(X)$.

On a alors :

(n5) pour tout $\lambda \in \mathcal{P}(X, \mathcal{A})$ et tout $f \in L^{1}\left(X, \mathcal{B}_{d}, \lambda\right)$, le quotient de dérivation $T_{r}(f \lambda, \lambda)$ tend vers $f \mathbf{I}_{X \backslash Z(\lambda, d)} \lambda$-presque sûrement sur $Y$ lorsque $r$ tend vers 0 .

Si de plus $\lambda^{*}(Z(\lambda, d))=0$ (ce qui est le cas "normal", voir 2.6), on a les deux propriétés suivantes :

(n6) pour tout $\lambda \in \mathcal{P}(X, \mathcal{A})$ et tout $f \in L^{1}\left(X, \mathcal{B}_{d}, \lambda\right)$, le quotient de dérivation $T_{r}(f \lambda, \lambda)$ tend vers $f \lambda$-presque sûrement sur $Y$ lorsque $r$ tend vers 0 ;

(n7) pour tout $\lambda \in \mathcal{P}(X, \mathcal{A})$ et tout $f \in L^{1}(X, \mathcal{A}, \lambda)$, le quotient de dérivation $T_{r}(f \lambda, \lambda)$ tend vers $\mathbb{E}_{\lambda}\left(f \mid \mathcal{B}_{d}\right) \lambda$-presque sûrement sur $Y$ lorsque $r$ tend vers 0 .

\section{Démonstration.}

Démonstration de $\left(n_{4}\right) \Longrightarrow(n 5)$. Pour tout $h \in L^{1}(X, \mathcal{A}, \lambda)$, on pose $U_{\lambda} h=\limsup _{r \rightarrow 0}\left|T_{r}(h \lambda, \lambda)-h \mathbf{I}_{X \backslash Z(\lambda, d)}\right|$.

Soit $f \in L^{1}\left(X, \mathcal{B}_{d}, \lambda\right)$; par $2.4 \mathrm{c}$, on peut se donner une fonction $g$ continue bornée sur $\left(X, \mathcal{T}_{d}\right)$ approchant $f$ à $\varepsilon$ près dans $L^{1}(X, \mathcal{A}, \lambda)$. Par continuité, on a $U_{\lambda} g=0$. Rappelons que $S_{1}=\sup _{0<u<1} T_{u}$.

Soit $k=f-g$. On a $\|k\|_{L^{1}}<\varepsilon$ et $U_{\lambda} k \leq S_{1}(|k| \lambda, \lambda)+|k|$. On a donc :

$$
U_{\lambda} f \leq U_{\lambda} g+U_{\lambda} k \leq S_{1}(|k| \lambda, \lambda)+|k| .
$$

Pour $\alpha>0$, on trouve $\left\{U_{\lambda} f>\alpha\right\} \subset\left\{S_{1}(|k| \lambda, \lambda)>\alpha / 2\right\} \cup\{|k|>\alpha / 2\}$, donc :

$$
\begin{aligned}
\alpha \lambda^{*}\left(Y \cap\left\{U_{\lambda} f>\alpha\right\}\right) & \leq \alpha \lambda^{*}\left(Y \cap\left\{S_{1}(|k| \lambda, \lambda)>\alpha / 2\right\}\right)+\alpha \lambda^{*}(Y \cap\{|k|>\alpha / 2\}) \\
& \leq 2 m\|k\|_{L^{1}}+2\|k\|_{L^{1}} \leq 2(m+1) \varepsilon
\end{aligned}
$$

(en utilisant l'inégalité maximale (n4) et l'inégalité de Tchebychev). 
On a donc $\lambda^{*}\left(Y \cap\left\{U_{\lambda} f>\alpha\right\}\right)=0$ en faisant tendre $\varepsilon$ vers 0 . En faisant décroître $\alpha$ vers 0 , on trouve que $Y \cap\left\{U_{\lambda} f>0\right\}$ est $\lambda$-négligeable.

Démonstration de (n6). Ce point est évident.

Démonstration de $(n 7)$. Soit $f \in L^{1}(X, \mathcal{A}, \lambda)$, on pose $f_{1}=\mathbb{E}_{\lambda}\left(f \mid \mathcal{B}_{d}\right)$ et $\nu_{1}=f_{1} \lambda$. On a $\nu\left(\mathbf{B}_{d}(y, r)\right)=\nu_{1}\left(\mathbf{B}_{d}(y, r)\right)$ pour tout $y$ et tout $r$. Donc $T_{r}(f \lambda, \lambda)=T_{r}\left(f_{1} \lambda, \lambda\right)$ tend vers $f_{1} \lambda$-presque sûrement sur $Y$ lorsque $r$ tend vers 0 .

Il reste à traiter le cas où $\nu$ peut avoir une partie étrangère à $\lambda$ :

Proposition 4.5. Soit $X$ un ensemble muni d'un noyau symétrique quelconque d avec $\mathcal{A}_{d} \subset \mathcal{B}_{d}$, et soit $Y \subset X$; soit $\mathcal{A}$ une tribu sur $X$ avec $\mathcal{A} \supset \mathcal{B}_{d}$. Soit $m$ un entier $\geq 1$. On suppose que $(X, d)$ possède la $\operatorname{prf}(m)$ restreinte aux boules centrées dans $Y$. On a alors:

(a) pour tout $\lambda \in \mathcal{P}(X, \mathcal{A})$, tout $\nu \in \mathcal{M}_{1}^{+}(X, \mathcal{A})$, tout $B \in \mathcal{B}_{d}$ et tout $\alpha>0$, on $a:$

$$
\alpha \lambda^{*}\left(Y \cap B \cap\left\{\limsup _{r \longrightarrow 0} T_{r}(\nu, \lambda)>\alpha\right\}\right) \leq m \nu(B)
$$

(b) soient $\lambda \in \mathcal{P}\left(X, \mathcal{B}_{d}\right)$ et $\nu \in \mathcal{M}_{1}^{+}\left(X, \mathcal{B}_{d}\right)$ étrangère à $\lambda$; alors $T_{r}(\nu, \lambda)$ converge vers $0 \lambda$-presque sûrement sur $Y$ lorsque $r$ tend vers 0 .

Démonstration. (a) Soient $\lambda \in \mathcal{P}(X, \mathcal{A}), \nu \in \mathcal{M}_{1}^{+}(X, \mathcal{A}), B \in \mathcal{B}_{d}$ et $\alpha>0$. On pose $S=\lim _{r \longrightarrow 0} S_{r}(\nu, \lambda)=\limsup _{r \longrightarrow 0} T_{r}(\nu, \lambda)$.

Soit $\varepsilon>0$. Appliquant 2.4a, on choisit $V \in \mathcal{B}_{d}$ ouvert avec $B \subset V$ et $\nu(V \backslash B)<\varepsilon$. On applique (n4) en remplaçant $\nu$ et $\lambda$ par leurs traces $\lambda_{V}$ et $\nu_{V}$ sur $V$, ce qui donne :

$\alpha \lambda^{*}\left(Y \cap V \cap\left\{S_{r}\left(\nu_{V}, \lambda_{V}\right)>\alpha\right\}\right) \leq m \nu(V) \leq m \nu(B)+m \varepsilon$ pour tout $r>0 ;$

On a donc $\alpha \lambda^{*}\left(Y \cap B \cap\left\{\lim _{r \longrightarrow 0} S_{r}\left(\nu_{V}, \lambda_{V}\right)>\alpha\right\}\right) \leq m \nu(B)+m \varepsilon$. Or $\lim _{r \longrightarrow 0} S_{r}\left(\nu_{V}, \lambda_{V}\right)=S$. Donc $\alpha \lambda^{*}(Y \cap B \cap\{S>\alpha\}) \leq m \nu(B)+m \varepsilon$. En faisant tendre $\varepsilon$ vers 0 , on obtient :

$$
\alpha \lambda^{*}(Y \cap B \cap\{S>\alpha\}) \leq m \nu(B) .
$$

(b) Soit $B \in \mathcal{B}_{d}$ avec $\lambda(B)=1$ et $\nu(B)=0$. Appliquant (a) à $B$, on trouve qu'on a :

$$
\alpha \lambda^{*}(Y \cap B \cap\{S>\alpha\})=0 \text { pour tout } \alpha>0 .
$$

On a donc $S=0 \lambda$-presque sûrement sur $Y$. 
(4.6) Soit $X$ un ensemble muni d'un noyau symétrique quelconque $d$ avec $\mathcal{A}_{d} \subset \mathcal{B}_{d} ;$ soit $\mathcal{A}$ une tribu sur $X$ avec $\mathcal{A} \supset \mathcal{B}_{d}$, et soit $Y \in \mathcal{A}$.

Soit $(X, d)$ possédant la $\operatorname{prf}(m)$ restreinte aux boules centrées dans $Y$; par 3.1 et en se restreignant aux boules de rayon $r$, on voit qu'on a $\mathbb{E}_{\lambda}^{*}\left(\left|T_{r}(\nu, \lambda)\right| \mathbf{I}_{Y}\right) \leq m|\nu|(X)$ pour tout $\lambda \in \mathcal{P}(X, \mathcal{A})$ et tout $\nu \in \mathcal{M}_{1}(X, \mathcal{A})$.

Dans le théorème 4.4 , la convergence des $T_{r}(f \lambda, \lambda)$ vers $\mathbb{E}_{\lambda}\left(f \mid \mathcal{B}_{d}\right)$ a donc lieu aussi en mesure et dans $L^{1}(Y, \mathcal{A}, \lambda)$ avec les hypothèses de mesurabilité convenables (et même dans $L^{p}(Y, \mathcal{A}, \lambda), p>1$ si $f \in L^{p}(X, \mathcal{A}, \lambda)$ ).

\section{(4.7) Dimension de Nagata}

Soit $X$ un ensemble muni d'un noyau symétrique $d$.

(a) On dit que l'espace $(X, d)$ est de dimension de Nagata $\leq n$ si, pour tout $a \in X$ et tous $y_{i} \in X(i=1, \ldots, n+2)$, il existe $i, j$ distincts avec $d\left(y_{i}, y_{j}\right) \leq d\left(a, y_{i}\right) \vee d\left(a, y_{j}\right)$

(b) Il résulte de 4.2 que $(X, d)$ possède la propriété de recouvrement faible de degré $\leq n+1$ si et seulement si $(X, d)$ est de dimension de Nagata $\leq n$.

(c) Le cas $n=0$ correspond aux ultramétriques.

En particulier, soit $\left(\mathcal{A}_{n}\right)_{n \in \mathbb{N}}$ une suite croissante de tribus sur $X$, chaque $\mathcal{A}_{n}$ étant engendré par une partition dénombrable ; on peut définir un écart ultramétrique $d$ à valeurs dans $2^{-\mathbb{N}}$ tel que les atomes de $\mathcal{A}_{n}$ soient exactement les boules fermées de rayon $2^{-n}$ pour $d$. Soit $\mathcal{A}$ une tribu sur $X$ contenant tous les $\mathcal{A}_{n}$.

Soient $\lambda \in \mathcal{P}(X, \mathcal{A})$ et $\nu \in \mathcal{M}_{1}^{+}(X, \mathcal{A})$; on a alors $T_{2^{-n}}(\nu, \lambda)=$ $\sum_{B} \mathbf{I}_{B} \nu(B) / \lambda(B)$ (où la somme est prise sur tous les atomes de $\mathcal{A}_{n}$ ) ; en particulier, pour $f \in L^{1}(X, \mathcal{A}, \lambda)$, on a $T_{2^{-n}}(f \lambda, \lambda)=\mathbb{E}_{\lambda}\left(f \mid \mathcal{A}_{n}\right) \lambda$-presque sûrement, et le théorème 4.4 n'est autre que le théorème de convergence presque sûre des martingales de la forme $f_{n}=\mathbb{E}_{\lambda}\left(f \mid \mathcal{A}_{n}\right)$ (martingales équi-intégrables, voir [27, p. 117]).

(d) Une borne inférieure de $n+1$ ultramétriques est toujours de dimension de Nagata $\leq n$; cela peut être mis à profit pour construire un noyau $d$ de dimension de Nagata $n$ bi-Lipschitz équivalent à la distance usuelle lorsque $(X, d)$ est $\mathbb{R}^{n}$ ou même un polyèdre fini de dimension $n$ inclus dans $\mathbb{R}^{N}$; on utilise pour cela, dans le premier cas des tribus dont les atomes sont des cubes, et dans le second cas des tribus liées aux subdivisions de Whitney (voir [5]).

Il est à noter que, sur $\mathbb{R}^{n}(n \geq 2)$, les distances associées à des normes sont de dimension de Nagata finie mais $>n$ (voir 4.9). 
(e) Soient $d_{1}$ et $d_{2}$ des noyaux symétriques tels que $\left(X, d_{i}\right)(i=1,2)$ possède la propriété de recouvrement faible de degré $\leq n_{i}$; soient $d=d_{1} \wedge d_{2}$ et $n=n_{1}+n_{2}$. On a alors :

(e.1) $(X, d)$ possède la propriété de recouvrement faible de degré $\leq n$;

(e.2) si, pour une même topologie $\mathcal{T}$ sur $X$, on a

$$
\mathcal{A}_{d_{1}} \subset \mathcal{B}(\mathcal{T}), \quad \mathcal{A}_{d_{2}} \subset \mathcal{B}(\mathcal{T}) \quad \text { et } \quad \mathcal{T} \subset \mathcal{T}_{d_{1}}, \mathcal{T} \subset \mathcal{T}_{d_{2}}
$$

alors $\mathcal{A}_{d} \subset \mathcal{B}(\mathcal{T})$ et $\mathcal{T} \subset \mathcal{T}_{d}$, donc $\mathcal{A}_{d} \subset \mathcal{B}_{d}$

(e.3) on peut choisir $d_{1}$ et $d_{2}$ de façon que $(X, d)$ ne possède la propriété de recouvrement forte de degré $\leq N$ pour aucun $N$ (et ceci même si $d_{1}$ et $d_{2}$ possèdent la propriété de recouvrement forte) :

pour cela, on prend deux ultramétriques $d_{1}$ et $d_{2}$ telles que toute boule ouverte pour $d_{1}$ coupe toute boule ouverte pour $d_{2}$ (par exemple en prenant $d_{1}$ et $d_{2}$ "indépendants" pour une loi $\lambda$ sur $(X, \mathcal{A}))$.

(f) La dimension de Nagata est supérieure ou égale à celle de De Groot. Dans un espace métrique de dimension métrique finie, la dimension de De Groot est toujours finie (voir 3.5d), mais la dimension de Nagata ne l'est pas toujours :

le groupe de Heisenberg $\mathbb{H}_{3}$ peut être muni d'un noyau symétrique invariant à gauche, qui est une distance [12], de dimension de Nagata infinie [24], [38], bien que de dimension métrique finie.

(g) On verra par contre plus loin (en 4.9) que la dimension de Nagata est égale à la dimension de De Groot sur $\mathbb{R}^{n}$ muni d'une norme quelconque et plus généralement sur tout espace métrique à courbure $\geq 0$.

(h) L'équivalence bi-Lipschizienne ne conserve pas la finitude de la dimension de Nagata. Prenons par exemple $X=\mathbb{R}^{+}$et $d\left(t, t^{\prime}\right)=\frac{t+t^{\prime}}{3} \wedge\left|t-t^{\prime}\right|$ pour tous $t, t^{\prime} \in X$; le noyau $d$ est une distance sur $X$ bi-Lipschitz équivalente à la distance usuelle $\left(t, t^{\prime}\right) \mapsto\left|t-t^{\prime}\right| ;(X, d)$ est donc de dimension métrique finie, donc de dimension de De Groot finie (voir 3.5d) ; mais la dimension de Nagata de $(X, d)$ est infinie (pour $a=0$ et $y_{i}=2^{-i}$, on a $d\left(y_{i}, y_{j}\right)>$ $d\left(a, y_{i}\right) \vee d\left(a, y_{i}\right)$ pour tous $i, j$ distincts $)$.

(i) On trouvait dans une note précédente de P. Assouad [2] une notion appelée aussi dimension de Nagata, mais en fait bien distincte (qu'on trouve utilisée notamment dans [25]) : cette dimension est $\leq n$ s'il existe $c>0$ tel que, pour tout $s>0$, on peut trouver un recouvrement $\mathcal{U}$ de $X$ par des ouverts de diamètre $\leq c s$ tel que tout ensemble de diamètre $\leq s$ rencontre au plus $n+1$ membres de $\mathcal{U}$.

Cette dimension n'intervient pas ici. 


\section{(4.8) Parties de dimension de Nagata finie}

Soit $X$ un ensemble muni d'un noyau symétrique $d$, et soit $Y \subset X$.

(a) On dit que $Y$ est une partie de dimension de Nagata $\leq n$ dans $(X, d)$ si, pour tout $a \in X$ et tous $y_{i} \in Y(i=1, \ldots, n+2)$, il existe $i, j$ distincts avec $d\left(y_{i}, y_{j}\right) \leq d\left(a, y_{i}\right) \vee d\left(a, y_{j}\right)$. Lorsque $Y=X$, on retrouve la définition 4.7a.

(b) Il résulte de 4.2 que $(X, d)$ possède la $\operatorname{prf}(n+1)$ restreinte aux boules centrées dans $Y$ si et seulement si $Y$ est de dimension de Nagata $\leq n$ dans $(X, d)$. On a alors :

(n5) pour tout $\lambda \in \mathcal{P}(X, \mathcal{A})$ et tout $f \in L^{1}\left(X, \mathcal{B}_{d}, \lambda\right)$, le quotient de dérivation $T_{r}(f \lambda, \lambda)$ tend vers $f \mathbf{I}_{X \backslash Z(\lambda, d)} \lambda$-presque sûrement sur $Y$ lorsque $r$ tend vers 0 ;

(c) Supposons qu'on ait seulement la $\operatorname{prf}(n+1)$ restreinte aux boules centrées dans $Y$ et de rayon $<s$; cela revient à dire que $Y$ est de dimension de Nagata $\leq n$ dans $(X, d \wedge s)$; dans ce cas (n5) subsiste.

(d) Supposons qu'on puisse trouver des $\left(Y_{k}\right)_{k \in \mathbb{N}}$ recouvrant $Y$, des nombres $\left(s_{k}\right)_{k \in \mathbb{N}}\left(s_{k} \in\right] 0,+\infty[)$ et des entiers $\left(N_{k}\right)_{k \in \mathbb{N}}$ tels qu'on ait la $\operatorname{prf}\left(N_{k}\right)$ restreinte aux boules centrées dans $Y_{k}$ et de rayon $<s_{k}$; alors (n5) subsiste. Il en résulte la partie directe du théorème de Preiss ci-dessous.

La dimension de Nagata intervient dans deux résultats importants :

Théorème de Nagata-Ostrand ([30], [31]). Un espace métrisable X est de dimension topologique $\leq n$ si et seulement sa topologie peut être définie par une distance $d$ telle que $(X, d)$ soit de dimension de Nagata $\leq n$.

On montre dans [5] qu'on peut remplacer dans l'énoncé précédent la distance $d$ par une borne inférieure de $(n+1)$ ultramétriques, évitant ainsi une bonne part des difficultés de la démonstration, qui tiennent à ce que $d$ doit être une distance.

Théorème de Preiss ([33]). Soit $(X, d)$ un espace métrique séparable complet; alors les assertions suivantes sont équivalentes :

(a) On peut trouver des $\left(Y_{k}\right)_{k \in \mathbb{N}}$ recouvrant $X$, des nombres $\left(s_{k}\right)_{k \in \mathbb{N}}\left(s_{k} \in\right.$ ] $0,+\infty[)$ et des entiers $\left(N_{k}\right)_{k \in \mathbb{N}}$ tels que, pour tout $k \in \mathbb{N}, Y_{k}$ soit de dimension de Nagata $\leq N_{k}$ dans $\left(X, d \wedge s_{k}\right)$;

(b) pour tout couple $\lambda, \nu$ de mesures de Radon positives et de masses totales finies $\operatorname{sur}(X, d)$ (avec $\nu=f \lambda)$, le quotient $T_{r}(\nu, \lambda)$ tend $\lambda$-presque partout vers $f$ lorsque $r$ tend vers 0 . 


\section{(4.9) Un calcul de la dimension de Nagata}

On montre enfin que, dans les espaces $(X, d)$ à courbure $\geq 0$ (et en particulier dans $\mathbb{R}^{n}$ ), il est facile d'évaluer le degré exact de la propriété de recouvrement faible. C'est l'object de la proposition suivante (qui vaudrait aussi pour les espaces à courbure $\leq 0)$.

Proposition 4.9.1. On suppose que $(X, d)$ est un espace métrique à courbure $\geq 0$ (par exemple $\mathbb{R}^{n}$ muni d'une norme quelconque). Alors les assertions suivantes sont équivalentes :

(a) pour tout $a \in X$ et tout $r \in] 0,+\infty[$, on peut trouver au plus $m$ points de $\mathbf{S}_{d}(a, r)$ de distances mutuelles $>r$;

(b) pour tous $a, y_{0}, \ldots, y_{m}$ dans $X$, il existe $i, j$ distincts avec

$$
d\left(y_{i}, y_{j}\right) \leq d\left(a, y_{i}\right) \vee d\left(a, y_{j}\right) .
$$

(c) pour tout $a \in X$ et tous $y_{0}, \ldots, y_{m} \in \mathbf{B}_{d}(a, r)$, il existe $i, j$ distincts avec $d\left(y_{i}, y_{j}\right) \leq r$.

En particulier (par 4.2) (X,d) possède la propriété de recouvrement faible de degré exactement $m$, où $m$ est le plus petit entier satisfaisant (a).

Démonstration. Démonstration de $(a) \Longrightarrow(b)$. Soient $a, y_{0}, \ldots, y_{m} \in X$. S'il existe $i$ avec $y_{i}=a$, on a $d\left(y_{i}, y_{j}\right)=d\left(a, y_{i}\right) \vee d\left(a, y_{j}\right)$ pour tout $j$. Sinon, pour chaque $i$ on choisit une fois pour toute un segment géodésique $\left[a, y_{i}\right]$ joignant $a$ à $y_{i}$.

(i) Soient $i, j$ distincts avec $d\left(y_{i}, y_{j}\right)>d\left(a, y_{i}\right) \vee d\left(a, y_{j}\right)$; on suppose $d\left(a, y_{i}\right) \leq d\left(a, y_{j}\right) ;$ on note $z_{j}$ le point du segment $\left[a, y_{j}\right]$ tel que $d\left(a, z_{j}\right)=$ $d\left(a, y_{i}\right)$; on a alors :

$$
\text { et } \quad \begin{aligned}
& d\left(y_{i}, y_{j}\right)>d\left(a, y_{j}\right) \\
& \text { et } \left.\quad y_{i}, z_{j}\right) \geq d\left(y_{i}, y_{j}\right)-d\left(y_{j}, z_{j}\right)>d\left(a, y_{j}\right)-d\left(y_{j}, z_{j}\right)=d\left(a, y_{i}\right) .
\end{aligned}
$$

Comme $(X, d)$ est à courbure $\geq 0$, il existe $\eta_{i j}>0$ tel qu'on ait $d\left(u_{i}(r), u_{j}(r)\right)>r$ pour tout $\left.r \in\right] 0, \eta_{i j}$ [ (où on note $u_{i}(r)$ le point du segment $\left[a, y_{i}\right]$ tel que $\left.d\left(a, u_{i}(r)\right)=r\right)$.

(ii) Supposons qu'on ait $d\left(y_{i}, y_{j}\right)>d\left(a, y_{i}\right) \vee d\left(a, y_{j}\right)$ pour tous $i, j$ distincts ; on pose alors $\eta=\inf _{i j} \eta_{i j}$ et on choisit $\left.t \in\right] 0, \eta[$; on a donc $d\left(u_{i}(t), u_{j}(t)\right)>t$ pour tous $i, j$ distincts dans $\{0, \ldots, m\}$, ce qui contredit (a).

Pour $X=\mathbb{R}^{n}$, on aurait pu prendre $t=1$ directement.

Démonstration de $(b) \Longrightarrow(c)$. Ce point est évident.

Démonstration de $(c) \Longrightarrow(a)$. Soient $a \in X$ et $y_{1}, \ldots, y_{k}$ des points de $\mathbf{S}_{d}(a, r)$ de distances mutuelles $>r$; on a alors $d\left(y_{i}, y_{j}\right)>r$ pour tous $i, j$ distincts, donc (c) implique que $k \leq m$. 
(4.9.2) Compte tenu de 3.5b, le Lemme précédent montre que, dans tout espace métrique $(X, d)$ à courbure $\geq 0$ (et en particulier dans $\mathbb{R}^{n}$ muni d'une norme quelconque) :

- la dimension de Nagata est égale à la dimension de De Groot,

- et $\mathbf{n}_{D}=\mathbf{n}_{C}$, i.e. la propriété de recouvrement faible de degré $\leq m$ restreinte aux boules à rayon constant implique la propriété de recouvrement faible de degré $\leq m$.

\section{(4.10) Une autre démonstration de la convergence presque sûre}

(4.10.1) Soit $X$ un ensemble muni d'un noyau symétrique $d$ avec $\mathcal{A}_{d} \subset \mathcal{B}_{d}$. Soit $\mathcal{A}$ une tribu sur $X$ avec $\mathcal{A} \supset \mathcal{B}_{d}$ et soit $\lambda \in \mathcal{P}(X, \mathcal{A})$. Soit $f$ une fonction $\mathcal{B}_{d}$-mesurable, partout finie et $\lambda$-intégrable de $X$ dans $\mathbb{R}$.

Comme en 3.7, on va voir qu'on peut tirer parti de la propriété (a) suivante démontrée en 2.4.3, afin de prouver la convergence presque sûre :

(a) pour tout $\varepsilon>0$, il existe une jauge $\gamma$ sur $X$ et une mesure $\mu \in$ $\mathcal{M}_{1}^{+}\left(X, \mathcal{B}_{d}\right)(\operatorname{avec} \mu(X) \leq 1)$ tels que :

$$
\left|f \lambda\left(\mathbf{B}_{d}(y, r)\right)-f(y) \lambda\left(\mathbf{B}_{d}(y, r)\right)\right| \leq \varepsilon \mu\left(\mathbf{B}_{d}(y, r)\right)
$$

pour tous $y \in X, r \in] 0, \gamma(y)[$.

(4.10.2) Si $(X, d)$ vérifie la propriété de recouvrement faible restreinte aux boules centrées en $Y$, on peut montrer à l'aide de (a) que :

$$
T_{r}(f \lambda, \lambda) \text { tend vers } f \mathbf{I}_{X \backslash Z(\lambda, d)} \quad \lambda \text {-presque partout dans } Y
$$

(on n'a plus besoin ici de savoir que, dans (a), $\gamma$ peut-être choisi $\lambda$-mesurable). Démonstration. On pose $Z=Z(\lambda, d)$. Soit $\varepsilon>0$; on se donne $\gamma$ et $\mu$ associés à $\varepsilon$ par (a).

On fixe $\alpha>0$; soit

$$
Y_{\alpha}=\left\{y \in Y\left|\limsup _{r \rightarrow 0}\right| T_{r}(f \lambda, \lambda)-f \mathbf{I}_{X \backslash Z} \mid>\alpha\right\} .
$$

Pour chaque $y \in Y_{\alpha}$, on choisit $r_{y}<\gamma(y)$ avec

$$
\left|T_{r_{y}}(f \lambda, \lambda)(y)-f(y) \mathbf{I}_{X \backslash Z}(y)\right|>\alpha
$$

(donc $y \notin Z$ ); quitte à l'augmenter un peu, on peut supposer que $r_{y}$ est rationnel. On pose $B(y)=\mathbf{B}_{d}\left(y, r_{y}\right)$ pour chaque $y \in Y_{\alpha}$.

On a donc, en utilisant (a) :

$$
\alpha \lambda(B(y))<|f \lambda(B(y))-f(y) \lambda(B(y))| \leq \varepsilon \mu(B(y)) .
$$

On considère $\mathbb{Q}$ comme une réunion croissante d'ensembles finis $D_{j}$ et on note $Y_{\alpha, j}$ l'ensemble des $y \in Y_{\alpha}$ avec $r_{y} \in D_{j}$. 
Fixons d'abord $j$; on peut extraire de $Y_{\alpha, j}$ une famille de points $\left(y_{i}\right)_{i \in I}$ telle que la famille $\left(B\left(y_{i}\right)\right)_{i \in I}$ recouvre $Y_{\alpha, j}$ et soit d'ordre $\leq m$; or $\lambda$ charge chaque $B\left(y_{i}\right)$ pour $i \in I$; donc $I$ est dénombrable, par 2.5.1. On a donc:

$$
\begin{aligned}
\alpha \lambda^{*}\left(Y_{\alpha, j}\right) & \leq \alpha \sum_{i \in I} \lambda\left(B\left(y_{i}\right)\right)<\varepsilon \sum_{i \in I} \mu\left(B\left(y_{i}\right)\right) \\
& \leq \varepsilon m \mu\left(\cup_{i \in I} B\left(y_{i}\right)\right) \leq \varepsilon m \mu(X) .
\end{aligned}
$$

En faisant tendre $\varepsilon$ vers 0 , on obtient $\lambda^{*}\left(Y_{\alpha, j}\right)=0$. En faisant varier $j$, on en déduit qu'on a $\lambda^{*}\left(Y_{\alpha}\right)=0$.

(4.10.3) La démonstration précédente s'appliquerait encore si on remplaçait $f \lambda$ par une fonction d'ensemble $\nu$ définie seulement sur les boules fermées de $(X, d)$ et vérifiant :

(a) pour tout $\varepsilon>0$, il existe une jauge $\gamma$ sur $X$ et une mesure $\mu \in$ $\mathcal{M}_{1}^{+}\left(X, \mathcal{B}_{d}\right)(\operatorname{avec} \mu(X) \leq 1)$ tels que :

$$
\left|\nu\left(\mathbf{B}_{d}(y, r)\right)-f(y) \lambda\left(\mathbf{B}_{d}(y, r)\right)\right| \leq \varepsilon \mu\left(\mathbf{B}_{d}(y, r)\right)
$$

pour tous $y \in X, r \in] 0, \gamma(y)[$;

(b) $\nu$ est "suradditive" au sens suivant (comme en 2.5.2) :

il existe $c \in] 0,+\infty\left[\right.$ tel que, pour toute famille finie $\mathcal{B}=\left(B_{i}\right)_{i \in I}$ de boules fermées, on a $\sum_{i \in I} \nu\left(B_{i}\right) \leq c o(\mathcal{B})$;

(c) il existe une fonction $\mathcal{A}$-mesurable positive $g$ avec $\nu(B) \geq-g \lambda(B)$ pour tout $B$ boule fermée.

La démonstration est semblable, à cela près que les boules $B(y)$ sont chargées par $2 g \lambda+\nu$ (et non par $\lambda)$.

Une remarque analogue vaudrait pour la convergence en mesure, en supposant cette fois que $\gamma$ est $\lambda$-mesurable.

\section{Propriété de recouvrement forte dans les espaces à courbure $\geq 0$}

On se propose de donner des évaluations précises du degré de la propriété de recouvrement forte dans $\mathbb{R}^{n}$ ou dans un espace à courbure $\geq 0$. On trouvera ces évaluations en 5.3 (précédés de lemmes techniques assez connus en 5.2) ; si on veut une démonstration assez rapide (mais avec de moins bonnes constantes) de la propriété de recouvrement forte dans $\mathbb{R}^{n}$, on peut se contenter de lire 5.2.2a, 5.2.3 et 5.3.5.

On étudie ensuite en 5.4 le cas de $\mathbb{R}$ et de $\mathbb{R}^{2}$.

Un résumé des résultats se trouve en 5.6. 
(5.1) Soit $\left(\mathbf{B}_{d}\left(y_{i}, r_{i}\right)\right)_{i \in I}$ une famille de boules fermées de $(X, d)$ et soit $E \subset I$. Lorsqu'on considèrera une telle famille, on posera parfois $B_{i}=$ $\mathbf{B}_{d}\left(y_{i}, r_{i}\right)$ pour alléger les notations. De plus, on attribuera souvent par abus de langage à $E$ les propriétés vérifiées par $\left(\mathbf{B}_{d}\left(y_{i}, r_{i}\right)\right)_{i \in E}$.

(a) Soit $\tau \geq 1$; la famille $\left(\mathbf{B}_{d}\left(y_{i}, r_{i}\right)\right)_{i \in E}$ est dite $\tau$-contrôlée si on a :

$\left(y_{j} \notin B_{i}\right.$ et $\left.r_{i} \geq r_{j} / \tau\right)$ ou $\left(y_{i} \notin B_{j}\right.$ et $\left.r_{j} \geq r_{i} / \tau\right)$, pour tous $i, j \in E$ distincts.

On note $\mathbf{o}(\tau)$ le maximum de $o(\mathcal{B})$ pour toutes les familles $\mathcal{B} \tau$-contrôlées de boules fermées de $(X, d)$; c'est en fait le maximum de $o(\mathcal{B})$ pour toutes les familles $\mathcal{B} \tau$-contrôlées finies. On note $\chi(\tau)$ le maximum de $\chi(\mathcal{B})$ pour toutes les familles $\mathcal{B} \tau$-contrôlées de boules fermées de $(X, d)$ à rayons bornés (si $\tau>1$ ) ou discrets (si $\tau=1$ ).

Rappelons que l'ordre $o(\mathcal{B})$ et l'indice de coloration $\chi(\mathcal{B})$ d'une famille $\mathcal{B}$ de parties de $X$ ont été définis en 1.1.

Evidemment $\mathbf{o}(\tau)$ et $\chi(\tau)$ sont des fonctions croissantes de $\tau$ et on a $\mathbf{o}(\tau) \leq \chi(\tau)$ pour tout $\tau \geq 1$.

(b) La famille $\left(\mathbf{B}_{d}\left(y_{i}, r_{i}\right)\right)_{i \in E}$ est 1-contrôlée si et seulement si elle vérifie :

$$
\left(y_{j} \notin B_{i}\right) \text { et }\left(y_{i} \notin B_{j}\right) \text {, pour tous } i, j \in I \text { distincts, }
$$

autrement dit $d\left(y_{i}, y_{j}\right)>r_{i} \vee r_{j}$ pour tous $i, j \in I$ distincts.

On dit alors que cette famille est sans liaison (voir 4.1).

(c) Soit $\tau \geq 1$; la famille $\left(\mathbf{B}_{d}\left(y_{i}, r_{i}\right)\right)_{i \in E}$ est appelée une $\tau$-configuration de centre $\ell$ (pour un $\ell \in E$ ) si on a :

$$
B_{i} \cap B_{\ell} \neq \varnothing \text { et } r_{i} \geq r_{\ell} / \tau \text { pour tout } i \in E \backslash\{\ell\} .
$$

On note $\mathbf{c}(\tau)$ le cardinal maximum d'une $\tau$-configuration dans une partie $\tau$-contrôlée ; $\mathbf{c}(\tau)$ est évidemment une fonction croissante de $\tau$.

(d) On note $\mathbf{c}\left(1^{+}\right)$(resp. o( $\left.\left.1^{+}\right)\right)$la limite de $\mathbf{c}(\tau)$ (resp. de $\left.\mathbf{o}(\tau)\right)$ lorsque $\tau$ tend vers $1(\tau>1)$. Par ailleurs, on renvoie à 1.2 pour les définitions de $\mathbf{n}_{C}$, $\mathbf{n}_{D}, \mathbf{n}_{B}$ et $\mathbf{N}_{C}, \mathbf{N}_{D}, \mathbf{N}_{B}$.

\section{(5.2) Familles contrôlées et configurations}

La proposition suivante contient des résultats d'extraction qui sont tout à fait classiques (voir par exemple [20]), et dont nous avons seulement un peu simplifié la démonstration (en la ramenant à l'extraction de "réseaux", c'est à dire de parties libres maximales dans certains graphes). 
Proposition 5.2.1. Soit $X$ un ensemble muni d'un noyau symétrique d et soit $\mathcal{B}=\left(\mathbf{B}_{d}\left(y_{i}, r_{i}\right)\right)_{i \in I}$ une famille à rayons bornés. On pose $B_{i}=\mathbf{B}_{d}\left(y_{i}, r_{i}\right)$ pour tout $i \in I$.

(a) Soit $\tau>1$. On peut extraire de $I$ une partie $F \tau$-contrôlée telle que $\cup_{j \in F} B_{j}$ recouvre $\left\{y_{i}\right\}_{i \in I}$. On a donc $\mathbf{n}_{B} \leq \mathbf{o}(\tau)$ et $\mathbf{N}_{B} \leq \chi(\tau)$.

(b) Si de plus $\mathcal{B}$ est à rayons discrets, on peut extraire de $I$ une partie $F$ sans liaison telle que $\cup_{j \in F} B_{j}$ recouvre $\left\{y_{i}\right\}_{i \in I}$. On a donc $\mathbf{N}_{D} \leq \chi(1)$.

Démonstration. Une partie $E$ de $I$ est dite discernable si on a $\left(y_{j} \notin B_{i}\right)$ ou $\left(y_{i} \notin B_{j}\right)$, pour tous $i, j \in E$ distincts. Si $r_{i}$ est constant pour $i \in E$, "discernable" est identique à "sans liaison".

Soit $R=\sup _{i \in I} r_{i}$ et $\tau>1$; pour chaque $k \in \mathbb{N}$, on pose $u_{k}=\tau^{-k} R$. Pour chaque $i \in I$, on note $\widetilde{r}_{i}$ la borne supérieure des $u_{k}<r_{i}$.

(a) Soit $E_{1}$ l'ensemble des $i \in I$ tels que $\widetilde{r}_{i}=u_{1}$; soit $F_{1}$ une partie discernable maximale de $E_{1}$. Supposons $F_{1}, \ldots, F_{k-1}$ définis ; soit $E_{k}$ l'ensemble des $i \in I$ tels que $\widetilde{r}_{i}=u_{k}$ et que $y_{i}$ ne soit dans aucun des $B_{j}$ pour $j \in F_{1} \cup \ldots \cup F_{k-1}$; soit $F_{k}$ une partie discernable maximale de $E_{k}$. La réunion des $F_{k}$ est la partie $F$ cherchée.

(b) On suppose maintenant que les $r_{i}$ appartiennent à la suite décroissante $\left(v_{k}\right)_{k \geq 1}$. On reprend la même démonstration en remplaçant $\widetilde{r}_{i}=u_{k}$ par $r_{i}=v_{k}($ voir 4.2).

Proposition 5.2.2. Soit $X$ un ensemble muni d'un noyau symétrique $d$. On a $\chi(\tau) \leq \mathbf{c}(\tau)$ pour tout $\tau \geq 1$.

Démonstration. Soit $\mathcal{B}=\left(\mathbf{B}_{d}\left(y_{i}, r_{i}\right)\right)_{i \in I}$ une famille de boules fermées. On pose $B_{i}=\mathbf{B}_{d}\left(y_{i}, r_{i}\right)$ pour tout $i \in I$. Soient $E \subset I$ et $\tau \geq 1$; une partie $F$ de $E$ est dite $\tau$-dense dans $E$ si, pour tout $i \in E$, il existe $j \in F$, avec $B_{i} \cap B_{j} \neq \varnothing$ et $r_{j} \geq r_{i} / \tau$. Si $\tau>1$, on définit $R, u_{k}=\tau^{-k} R$ et $\widetilde{r}_{i}$ comme en 5.2.2.

(a) Soit $\tau>1$ (resp. $\tau=1$ ). Si $I$ est à rayons bornés (resp. discrets), montrons qu'on peut extraire de $E$ une partie $F \tau$-dense (resp. 1-dense) dans $E$, qui est 1-colorable.

Soit $E_{1}$ l'ensemble des $i \in E$ tels que $\widetilde{r}_{i}=u_{1}$ (resp. $\left.r_{i}=v_{1}\right)$; soit $F_{1}$ une partie 1-colorable maximale de $E_{1}$. Supposons $F_{1}, \ldots, F_{k-1}$ définis ; soit $E_{k}$ l'ensemble des $i \in E$ tels que $\widetilde{r}_{i}=u_{k}$ (resp. $r_{i}=v_{k}$ ) et que $B_{i}$ ne coupe aucun des $B_{j}$ pour $j \in F_{1} \cup \ldots \cup F_{k-1}$; soit $F_{k}$ une partie 1-colorable maximale de $E_{k}$. La réunion des $F_{k}$ est la partie $F$ cherchée.

(b) Soit $\tau \geq 1$. Toute partie $\tau$-contrôlée de $I$ est $\mathbf{c}(\tau)$-colorable.

On pose $N=\mathbf{c}(\tau)$. Soit $E$ une partie $\tau$-contrôlée de $I$; soit $E_{1}$ une partie de $E$ 1-colorable et $\tau$-dense dans $E$; soit $E_{2}$ une partie de $E \backslash E_{1}$ 1-colorable et $\tau$-dense dans $E \backslash E_{1}$, et ainsi de suite. 
Les parties $E_{1}, \ldots, E_{N}$ recouvrent $E$; en effet, si $\ell$ était un élément de $E \backslash\left(E_{1} \cup \ldots \cup E_{N}\right)$, on pourrait trouver pour chaque $p=1, \ldots, N$ un élément $j \in E_{p}$ tel que $B_{j} \cap B_{\ell} \neq \varnothing$ et $r_{j} \geq r_{\ell} / \tau$, ce qui formerait une $\tau$-configuration de cardinal $>\mathbf{c}(\tau)$, ce qui est impossible.

\section{(5.3) Evaluations du degré dans la propriété de recouvrement forte}

(5.3.1) Quelques évaluations de $\mathbf{c}(\tau)$ dans $\mathbb{R}^{n}$

(a) La démonstration de Besicovitch $[6]\left(\right.$ dans $\left.\mathbb{R}^{2}\right)$ consiste à majorer d'abord $\mathbf{c}(1)$ :

soit $\left(\mathbf{B}_{d}\left(y_{i}, r_{i}\right)\right)_{i \in E}$ une 1-configuration 1-contrôlée de centre $\ell$ dans $\mathbb{R}^{2}$; on pose $a=y_{\ell}$ et $r=r_{\ell}$; on note $u_{i}=\frac{y_{i}-a}{\left\|y_{i}-a\right\|}$ pour tout $i \neq \ell$; on partage $I$ en $J=\left\{i \in E \mid d\left(a, y_{i}\right) \leq \frac{3}{2} r\right\}$ et $K=E \backslash J$; pour $i, j$ distincts dans $J$ (resp. dans $K$ ), on montre que $d\left(u_{i}, u_{j}\right)$ est minoré par $2 / 3$ (resp. par $1 / 2$ ), donc cardinal $(J) \leq 9$ (resp. $\operatorname{cardinal}(K) \leq 12)$.

On passe ensuite à $\mathbf{c}\left(1^{+}\right)$d'une façon qui n'est pas complètement explicite.

(b) La plupart des démonstrations ultérieures (par exemple [20] et [28]) consistent à considérer une $\tau$-configuration $\left(\mathbf{B}_{d}\left(y_{i}, r_{i}\right)\right)_{i \in E}$ de centre $\ell$ et à partager $E$ en $J=\left\{i \in E \mid d\left(a, y_{i}\right) \leq \alpha r\right\}$ et $K=E \backslash J$, puis à majorer le cardinal de $J$ (resp. de $K$ ) grâce à la "doubling property" (resp. en minorant l'angle $\left(y_{i}, a, y_{j}\right)$ pour $i, j \in K$ distincts).

Le résultat suivant est en substance dans [6] :

Lemme 5.3.2. Soit $X$ un ensemble muni d'un noyau symétrique d. Soient $\eta \geq 0$ et $\tau=1+\eta$. Soit $\left(\mathbf{B}_{d}\left(y_{i}, r_{i}\right)\right)_{i \in E}$ une famille $\tau$-contrôlée dans $(X, d)$. On a alors $d\left(y_{i}, y_{j}\right)>r_{i} \vee r_{j}-\eta\left(r_{i} \wedge r_{j}\right)$ pour tous $i, j \in E$ distincts.

Démonstration. Soient $i, j$ distincts dans $E$; quitte à échanger $i$ et $j$, on a $d\left(y_{i}, y_{j}\right)>r_{i} \geq r_{j} / \tau$; on a évidemment $r_{i} \geq r_{i}-\eta r_{i}$ et $r_{i} \geq r_{i}-\eta r_{j}$; de plus on a $r_{i} \geq r_{j}-\eta r_{i}\left(\right.$ resp. $\left.r_{i} \geq r_{j}-\eta r_{j}\right)$, car $1+\eta=\tau($ resp. $1-\eta \leq 1 / \tau)$.

Furedi et Loeb [21] ont montré que, dans $\mathbb{R}^{n}$ muni d'une norme quelconque, $\mathbf{c}\left(1^{+}\right)$est le nombre maximum de points de distances mutuelles $\geq t$ dans une boule fermée quelconque de rayon $2 t$, l'un des points étant le centre (Sullivan [37] a montré le même résultat pour $\mathbb{R}^{n}$ euclidien).

Cela vaut aussi dans les espaces à courbure $\geq 0$ (et même $\leq 0)$ :

Proposition 5.3.3. Soit $(X, d)$ un espace métrique à courbure $\geq 0$. Soient $\eta \geq 0, \tau=1+\eta$ et $\delta=2 \eta \tau$. Alors $\mathbf{c}(\tau)$ est majoré par le nombre maximum $m_{\delta}$ de points de distances mutuelles $>(1-\delta) t$ dans une boule fermée quelconque de rayon $2 t$, l'un des points étant le centre. 
Démonstration. Soit $B_{j}=\mathbf{B}_{d}\left(y_{j}, r_{j}\right)(j \in E)$ une $\tau$-configuration de centre $\ell$ dans une partie $\tau$-contrôlée. Posons $a=y_{\ell}$ et $r=r_{\ell}$.

Pour chaque $j \in E \backslash\{\ell\}$, on choisit un segment géodésique $\left[a, y_{j}\right]$ reliant $a$ à $y_{j}$, et on note $z_{j, t}$ le point de $\left[a, y_{j}\right]$ à la distance $\frac{t}{r}\left[d\left(a, y_{j}\right) \wedge 2 r\right]$ de $a$. On a alors :

$$
\begin{aligned}
d\left(a, z_{j, t}\right) & \leq 2 t, \quad d\left(a, z_{j, t}\right) \geq t / \tau, \quad d\left(a, y_{j}\right) \leq r_{j}+r, \\
d\left(y_{j}, z_{j, r}\right) & =d\left(a, y_{j}\right)-2 r<r_{j}-r, \quad \text { dès que } d\left(a, y_{j}\right)>2 r .
\end{aligned}
$$

On pose $J=\left\{i \in E \mid d\left(a, y_{i}\right) \leq 2 r\right\}$ et $K=E \backslash J$.

(a) On se donne $i, k \in E \backslash\{\ell\}(i \neq k)$ avec $0<d\left(a, y_{i}\right) \leq d\left(a, y_{k}\right)$. On va montrer qu'il existe $s>0$ tel que $d\left(z_{i, s}, z_{k, s}\right)>(1-\delta) s$; pour cela, on distingue trois cas :

(a1) pour $i, k \in J$ distincts, on prend $s=r, z_{i, r}=y_{i}$ et $z_{k, r}=y_{k}$; on a alors :

$$
\begin{array}{rc}
d\left(z_{i, r}, z_{k, r}\right)=d\left(y_{i}, y_{k}\right) & \\
>r_{i} \wedge r_{k} \geq r / \tau & (\tau \text {-configuration }), \\
\geq(1-\delta) r & (\text { car } 1-1 / \tau=\eta / \tau \leq 2 \eta \tau=\delta) ;
\end{array}
$$

(a2) pour $i \in J, k \in K$, on prend $s=r, z_{i, r}=y_{i}$ et $z_{k, r}$ le point de $\left[a, y_{k}\right]$ à la distance $2 r$ de $a$; on a alors :

$$
\begin{array}{rlrl}
d\left(z_{i, r}, z_{k, r}\right) \geq d\left(y_{i}, y_{k}\right)-d\left(y_{k}, z_{k, r}\right) & & \\
& >\left(r_{k}-\eta r_{i}\right)-d\left(y_{k}, z_{k, r}\right) & & (\operatorname{par} 5.3 .2), \\
& >\left(r_{k}-\eta r_{i}\right)-\left(r_{k}-r\right) & & \left(\operatorname{car} d\left(y_{k}, z_{k, r}\right)<r_{k}-r\right), \\
& \geq(1-\delta) r & & \left(\operatorname{car} r_{i} \leq \tau d\left(a, y_{i}\right) \leq 2 \tau r\right) ;
\end{array}
$$

(a3) pour $i, k \in K$ distincts, on prend $s=d\left(a, y_{i}\right) / 2, z_{i, s}=y_{i}$ et $z_{k, s}$ le point de $\left[a, y_{k}\right]$ à la distance $d\left(a, y_{i}\right)$ de $a$; on a alors :

$$
\begin{array}{rlrl}
d\left(z_{i, s}, z_{k, s}\right) \geq d\left(y_{i}, y_{k}\right)-d\left(a, y_{k}\right)+d\left(a, y_{i}\right) & & \\
& >\left(r_{k}-\eta r_{i}\right)-\left(r_{k}+r\right)+d\left(a, y_{i}\right) & & (\operatorname{par} 5.3 .2), \\
& =s-\eta r_{i}-r+d\left(a, y_{i}\right) / 2 \geq s-\eta r_{i} & & \left(\operatorname{car} d\left(a, y_{i}\right) \geq 2 r\right), \\
& \geq s-\eta \tau d\left(a, y_{i}\right) & & \left(\operatorname{car} d\left(a, y_{i}\right) \geq r_{i} / \tau\right), \\
& \geq(1-\delta) s & & (\operatorname{car} \delta=2 \eta \tau) .
\end{array}
$$

(b) Comme $(X, d)$ est à courbure $\geq 0$, on obtient $d\left(z_{i, t}, z_{k, t}\right)>(1-\delta) t$ pour $t$ assez petit.

Pour $\eta=0$, la démonstration ci-dessus se simplifie manifestement et on obtient que $\mathbf{c}(1)$ est majoré par le nombre maximum $m_{0}$ de points de distances mutuelles $>t$ dans une boule fermée quelconque de rayon $2 t$, l'un des points étant le centre. 
(5.3.4) Soit $(X, d)$ un espace métrique géodésique.

(a) Soit $m_{0}^{+}$le nombre maximum de points de $\mathbf{B}_{d}(a, 2 t)$ de distances mutuelles $\geq t$ l'un des points étant $a$. On a évidemment $m_{0}^{+} \geq m_{0}$.

(b) On fixe $\tau>1$. Soit $\varepsilon>0$ avec $t-\varepsilon \geq(t+\varepsilon) / \tau$.

Soit $\left(y_{i}\right)_{i \in I}$ une famille de points de distances mutuelles $\geq t$ dans

$$
\mathbf{B}_{d}(a, 2 t) \backslash \mathbf{U}_{d}(a, t) .
$$

Les boules $\mathbf{B}_{d}(a, t+\varepsilon)$ et $\mathbf{B}_{d}\left(y_{i}, t-\varepsilon\right)$ (pour $i \in I$ ) forment une famille de boules $\tau$-controlée qui est une $\tau$-configuration.

On a donc $\mathbf{c}(\tau) \geq m_{0}^{+}$.

(c) Soit $\left(y_{i}\right)_{i \in I}$ une famille de points de distances mutuelles $>t$ dans $\mathbf{B}_{d}(a, 2 t) \backslash \mathbf{B}_{d}(a, t)$. Les boules $\mathbf{B}_{d}(a, t)$ et $\mathbf{B}_{d}\left(y_{i}, t\right)$ (pour $i \in I$ ) forment une famille de boules sans liaison qui est une 1-configuration.

On a donc $\mathbf{c}(1) \geq m_{0}$, et même (par 5.3.3) $\mathbf{c}(1)=m_{0}$ dès que $(X, d)$ est à courbure $\geq 0$.

(d) Lorsque $\delta>0$ décroît vers 0 , l'entier $m_{\delta}$ décroît et se stabilise sur une valeur limite.

Furedi et Loeb [21] ont montré que, dans $\mathbb{R}^{n}$, cette valeur limite est $m_{0}^{+}$; en effet, dans ce cas, on se ramène à $a=0$ et $t=1$, puis on utilise la compacité de $\mathbf{B}_{d}(0,2)$.

On a donc, dans $\mathbb{R}^{n}, \mathbf{c}\left(1^{+}\right)=m_{0}^{+}$.

(5.3.5) Quitte à obtenir une moins bonne évaluation de $c(\tau)$, on peut simplifier substantiellement l'argument donné en 5.3.3 en majorant séparément le cardinal de $J=\left\{i \in E \mid d\left(a, y_{i}\right) \leq 2 r\right\}$ et celui de $K=E \backslash J$. Voici ce que cela donne dans $\mathbb{R}^{n}$ muni de la norme $\|\cdot\|$ (quitte à faire une translation, on suppose $a=0)$ :

on se donne $i, k \in E \backslash\{\ell\}(i \neq k)$ avec $0<\left\|y_{i}\right\| \leq\left\|y_{k}\right\|$;

- pour $i, k \in J$, on a :

$$
\left\|y_{i}-y_{k}\right\|>r_{i} \wedge r_{k} \geq r / \tau \quad(\tau \text {-configuration }) ;
$$

- pour $i, k \in K$, on pose $\theta=1 /\left\|y_{i}\right\|, s=\left\|y_{i}\right\| / 2, z_{i}=y_{i} /\left\|y_{i}\right\|$ et $z_{k}=y_{k} /\left\|y_{k}\right\|$; on a alors :

$$
\begin{aligned}
\left\|z_{i}-z_{k}\right\| & \geq\left\|z_{i}-\theta y_{k}\right\|-\left\|\theta y_{k}-z_{k}\right\| & & \\
& \geq \theta\left[\left\|y_{i}-y_{k}\right\|-\left\|y_{k}\right\|+\left\|y_{i}\right\|\right] & & \\
& >\theta\left[\left(r_{k}-\eta r_{i}\right)-\left(r_{k}+r\right)+\left\|y_{i}\right\|\right] & & (\operatorname{par} 5.3 .2), \\
& =\theta\left[s-\eta r_{i}-r+\left\|y_{i}\right\| / 2\right] \geq \theta\left[s-\eta r_{i}\right] & & \left(\operatorname{car}\left\|y_{i}\right\| \geq 2 r\right), \\
& \geq \theta\left[s-\eta \tau\left\|y_{i}\right\|\right] & & \left(\operatorname{car}\left\|y_{i}\right\| \geq r_{i} / \tau\right), \\
& \geq \theta(1-\delta) s=(1-\delta) / 2 & & (\operatorname{car} \delta=2 \eta \tau) .
\end{aligned}
$$


On majore le cardinal de $J$ (resp. de $K$ ) grâce à la compacité de la boule unité (resp. de la sphère unité) de $\mathbb{R}^{n}$.

Cependant, l'évaluation du degré de la propriété de recouvrement forte obtenue en 5.3.3 n'est pas optimale dans $\mathbb{R}^{n}$, comme on le verra ci-dessous.

\section{(5.4) Cas de $\mathbb{R}$ et de $\mathbb{R}^{2}$}

Proposition 5.4.1. Dans $\mathbb{R}$ muni de la distance usuelle, on a $\mathbf{N}_{B}=2$ (alors que $\mathbf{c}\left(1^{+}\right)=5$ ).

Démonstration. Soit $\mathcal{B}$ une famille de boules (intervalles) fermées de $\mathbb{R}$ à rayons bornés. La démonstration se fait en deux étapes :

(a) On note que $\mathbb{R}$ possède la propriété de recouvrement forte : en effet on a $\mathbf{N}_{B} \leq \mathbf{c}\left(1^{+}\right)=5$ (estimation a priori résultant de 5.2.1 et 5.2.2) ; on peut donc extraire de $\mathcal{B}$ une famille $\mathcal{E}$ d'ordre $\leq 5$ recouvrant les centres des éléments de $\mathcal{B}$.

(b) On peut montrer que, de toute famille $\mathcal{E}$ d'intervalles fermés de $\mathbb{R}$ d'ordre fini en tout point, on peut extraire deux parties $\mathcal{F}_{1}$ et $\mathcal{F}_{2}$ constituées d'intervalles disjoints et telles que $\mathcal{F}_{1} \cup \mathcal{F}_{2}$ recouvre $\cup_{B \in \mathcal{E}} B$.

Dans $\mathbb{R}^{2}$, nous n'avons de résultats précis que pour les familles de boules $\ell^{\infty}$ à rayon constant, c'est à dire pour les familles de carrés de côté constant :

Proposition 5.4.2. On se place dans $\mathbb{R}^{2}$ muni de la distance $d(x, y)=$ $\left|x_{1}-y_{1}\right| \vee\left|x_{2}-y_{2}\right|$ (correspondant à la norme $\ell^{\infty}$ ). On a alors $\mathbf{N}_{C}=5$ ou 6 . De façon précise on a :

(a) Toute famille $\left(\mathbf{B}_{d}\left(y_{i}, r_{i}\right)\right)_{i \in I} \grave{a}$ rayon constant et sans liaison est 6-colorable.

(b) On peut trouver une famille de 8 boules fermées à rayon constant, sans liaison, qui est 5-colorable, mais pas 4-colorable.

Démonstration. (a) On note $\pi_{1}$ la projection sur le premier axe de coordonnées. Quitte à faire une homothétie, on suppose $r=1$. Pour $q=0,1,2$, on note $X_{q}$ la réunion des bandes $Y_{p}=\mathbb{R} \times[p, p+1[\operatorname{avec} p=q(\bmod 3)$. A $p$ fixé, les boules $\mathbf{B}_{d}\left(y_{i}, r_{i}\right)$ dont le centre est dans $Y_{p}$ peuvent être coloriées en 2 couleurs, car leurs images par $\pi_{1}$ sont sans liaison dans $\mathbb{R}$. Il en résulte que, à $q$ fixé, les boules dont le centre est dans $X_{q}$ peuvent être coloriées en 2 couleurs (on colorie de façon indépendante dans chaque bande $Y_{p}$ contenue dans $X_{q}$ ), ce qui donne le résultat.

(b) On considère la famille $\mathcal{B}$ des 8 boules suivantes de rayon 8 et de centres respectifs :

$$
\begin{array}{rlll}
m=(0,0), & l=(0,-9), & i=(5,9), & i^{\prime}=(-5,9), \\
j=(14,2), & j^{\prime}=(-14,2), & k=(9,-7), & k^{\prime}=(-9,-7),
\end{array}
$$


La famille $\mathcal{B}$ est sans liaison ; les couples correspondant à des boules disjointes sont les suivants :

$$
\left(l, i^{\prime}\right),(l, i),\left(i^{\prime}, j\right),\left(i, j^{\prime}\right),\left(j, k^{\prime}\right),\left(j, j^{\prime}\right),\left(k, k^{\prime}\right),\left(k, j^{\prime}\right)
$$

ce qui permet de montrer aisément que $\mathcal{B}$ est 5-colorable, mais pas 4-colorable.

(5.4.3) Pour $\mathbb{R}^{n}$ muni de la norme $\ell_{\infty}$, on a, au vu de ce qui précède :

$\begin{array}{lllllll}n & \mathbf{n}_{D} & \mathbf{N}_{C} & \mathbf{N}_{D} & \mathbf{N}_{B} & \mathbf{c}(1) & \mathbf{c}(1+) \\ 1 & 2 & 2 & 2 & 2 & 3 & 5 \\ 2 & 4 & 5 \text { ou } 6 & ? & ? & 13 & 25 \\ k & 2^{k} & ? & ? & ? & ? & 5^{k}\end{array}$

Pour $\mathbb{R}^{n}$ muni de la norme euclidienne, on a :

$\begin{array}{lllllll}2 & 5 & ? & ? & ? & 13 & 19\end{array}$

Le résultat suivant précise un calcul fait au paragraphe 4 .

\section{(5.5) Evaluations du degré dans la propriété de De Giorgi}

On rappelle que $\mathbf{n}_{D}$ est le degré optimal pour la prf dans $(X, d)$ et que $\mathbf{n}_{B}$ est le degré optimal pour la prf étendue aux familles de boules à rayons bornés, dite propriété de De Giorgi (voir 1.2).

\section{Proposition 5.5.1.}

(a) Soit $X$ un ensemble muni d'un noyau symétrique $d$. On a alors $\mathbf{n}_{D}=$ $\mathbf{o}(1)$ et $\mathbf{n}_{B} \leq \mathbf{o}(\tau)$ (pour $\left.\tau>1\right)$.

(b) On suppose que $(X, d)$ est un espace métrique à courbure $\geq 0$; alors $\mathbf{o}(1)=n_{0}$, où $n_{0}$ le nombre maximal de points de distances mutuelles $>t$ sur une sphère de $(X, d)$ de rayon $t$ quelconque.

(c) On suppose que $(X, d)$ est un espace métrique à courbure $\geq 0$. Soient $\eta>0, \tau=1+\eta$ et $\delta=2 \eta \tau$. Alors $\mathbf{o}(\tau) \leq n_{\delta}+1$, où $n_{\delta}$ est le nombre maximal de points de distances mutuelles $>(1-\delta) t$ sur une sphère de $(X, d)$ de rayon $t$ quelconque.

Démonstration. L'assertion (a) ayant été démontré en 4.2 et 5.2 .1 et l'assertion (b) en 4.9.1, il reste à démontrer l'assertion (c) :

Soit $B_{i}=\mathbf{B}_{d}\left(y_{i}, r_{i}\right)(i \in I)$ une famille finie et $\tau$-contrôlée de boules fermées contenant un point $a$ de $X$. Pour chaque $i \in I\left(y_{i} \neq a\right)$, on choisit un segment géodésique $\left[a, y_{i}\right]$ reliant $a$ à $y_{i}$. 
(i) Soient $i, j$ distincts dans $I$; on suppose $d\left(a, y_{i}\right) \leq d\left(a, y_{j}\right)$. On a $2 d\left(a, y_{j}\right) \geq d\left(a, y_{i}\right)+d\left(a, y_{j}\right) \geq d\left(y_{i}, y_{j}\right)>r_{j} / \tau$. On note $z_{j}$ le point de $\left[a, y_{j}\right]$ situé à la distance $d\left(a, y_{i}\right)$ de $a$.

(ii) Soit $y_{\ell}$ tel que $d\left(a, y_{\ell}\right)=\inf _{i \in I} d\left(a, y_{i}\right)$. Soient $i, j$ distincts dans $I \backslash\{\ell\}$ avec $d\left(a, y_{i}\right) \leq d\left(a, y_{j}\right)$. On rappelle que $d\left(y_{i}, y_{j}\right) \geq r_{j}-\eta r_{i}$ (voir 5.3.2). On a alors :

$$
\begin{aligned}
d\left(y_{i}, z_{j}\right) & \geq d\left(y_{i}, y_{j}\right)-d\left(a, y_{j}\right)+d\left(a, y_{i}\right) \\
& >\left(r_{j}-\eta r_{i}\right)-r_{j}+d\left(a, y_{i}\right)=d\left(a, y_{i}\right)-\eta r_{i}
\end{aligned}
$$

en appliquant (i) au couple $\ell, i$, on obtient :

$$
d\left(a, y_{i}\right)-\eta r_{i}>(1-2 \eta \tau) d\left(a, y_{i}\right)=(1-\delta) d\left(a, y_{i}\right)
$$

(iii) Comme $(X, d)$ est à courbure $\geq 0$, il existe $\eta_{i j}>0$ tel qu'on ait $d\left(u_{i}(t), u_{j}(t)\right)>(1-\delta) t$ pour tous $i, j$ distincts dans $I \backslash\{\ell\}$ et tout $\left.t \in\right] 0, \eta_{i j}[$ (où on note $u_{i}(t)$ le point du segment $\left[a, y_{i}\right]$ tel que $d\left(a, u_{i}(t)\right)=t$ ).

On note $\eta$ la borne inférieure des $\eta_{i j}$ pour $i, j$ distincts dans $I \backslash\{\ell\}$ et on choisit $t \in] 0, \eta\left[\right.$; on a donc $d\left(u_{i}(t), u_{j}(t)\right)>(1-\delta) t$ pour tous $i, j$ distincts dans $I \backslash\{\ell\}$. D'où le résultat.

Pour $X=\mathbb{R}^{n}$, on aurait pu prendre $t=1$ directement.

(5.5.2) Soit $(X, d)$ un espace métrique géodésique.

(a) Soit $n_{0}^{+}$le nombre maximum de points de distances mutuelles $\geq t$ sur une sphère de $(X, d)$ de rayon $t$ quelconque. On a évidemment $n_{0}^{+} \geq n_{0}$ et $n_{0}=\mathbf{o}(1) \leq \mathbf{o}\left(1^{+}\right)$.

(b) Lorsque $\delta>0$ décroît vers 0 , l'entier $n_{\delta}$ décroît et se stabilise sur une valeur limite.

Dans $\mathbb{R}^{n}$, cette valeur limite est $n_{0}^{+}$; en effet, dans ce cas, on se ramène à $a=0$ et $t=1$, puis on utilise la compacité de $\mathbf{S}_{d}(0,1)$.

On a donc, dans $\mathbb{R}^{n}, \mathbf{o}\left(1^{+}\right) \leq n_{0}^{+}+1$.

\section{(5.6) Résumé des évaluations de degrés}

On note $\operatorname{pr} \mathbf{F}(m)$ pour "propriété de recouvrement forte de degré $\leq m$ ".

On rappelle que $\mathbf{N}_{B}$ est le degré optimal pour la $\mathbf{p r} \mathbf{F}$ dans $(X, d)$ et que $\mathbf{N}_{D}$ est le degré optimal pour la $\mathbf{p r} \mathbf{F}$ restreinte aux familles de boules à rayons discrets (voir 1.2).

Résumons en un seul énoncé les évaluations concernant $\mathbf{n}_{D}, \mathbf{n}_{B}, \mathbf{N}_{D}$, $\mathbf{N}_{B}$, démontrées en $5.2,5.3$ et 5.5 : 


\section{Proposition 5.6.1.}

(a) Soit $X$ un ensemble muni d'un noyau symétrique d; on a alors :

$$
\begin{aligned}
& \mathbf{n}_{D}=\mathbf{o}(1) \text { et } \mathbf{n}_{B} \leq \mathbf{o}(\tau) \text { pour tout } \tau>1 \\
& \mathbf{N}_{D} \leq \chi(1) \leq \mathbf{c}(1) \text { et } \mathbf{N}_{B} \leq \chi(\tau) \leq \mathbf{c}(\tau) \text { pour tout } \tau>1
\end{aligned}
$$

(b) Soit $(X, d)$ un espace métrique à courbure $\geq 0$, on a alors :

$$
\mathbf{o}(1)=n_{0},
$$

où $n_{0}$ est le nombre maximum de points de distances mutuelles $>t$ sur une sphère de $(X, d)$ de rayon $t$ quelconque.

$n_{0} \leq \mathbf{o}(\tau) \leq n_{\delta}+1$ pour tout $\tau>1$ (avec $\delta=2 \tau(\tau-1)$, où $n_{\delta}$ est le nombre maximum de points de distances mutuelles $>$ $(1-\delta) t$ sur une sphère de $(X, d)$ de rayon $t$ quelconque.

$\mathbf{c}(1)=m_{0}$,

où $m_{0}$ est le nombre maximum de points de distances mutuelles $>t$ dans une boule fermée de $(X, d)$ de rayon $2 t$ quelconque, l'un des points étant le centre.

$m_{0}^{+} \leq \mathbf{c}(\tau) \leq m_{\delta}$ pour tout $\tau>1$ (avec $\left.\delta=2 \tau(\tau-1)\right)$

où $m_{\delta}$ (resp. $m_{0}^{+}$) est le nombre maximum de points de distances mutuelles $>(1-\delta) t$ (resp. $\geq t)$ dans une boule fermée de $(X, d)$ de rayon $2 t$ quelconque, l'un des points étant le centre.

(c) Lorsque $(X, d)$ est $\mathbb{R}^{n}$ muni de la distance associée à une norme, on a :

$$
n_{0} \leq \mathbf{o}\left(1^{+}\right) \leq n_{0}^{+}+1
$$

où $n_{0}^{+}$est le nombre maximum de points de distances mutuelles $\geq t$ sur une sphère de $(X, d)$ de rayon $t$ quelconque.

$\mathbf{c}\left(1^{+}\right)=m_{0}^{+}$(ce dernier point est dî à Furedi et Loeb [21]).

\section{Dimension métrique et propriétés de recouvrement}

Dans ce paragraphe, on se propose de préciser les liens entre la propriété de recouvrement faible et la propriété de recouvrement forte, notamment lorsque $(X, d)$ est un espace métrique de dimension métrique finie.

Les résultats principaux de ce paragraphe se trouvent en 6.5 (avec un lemme important en 6.4) ; mais des préliminaires $(6.1,6.2,6.3)$ permettent d'abord de présenter le cadre géométrique de ces résultats. 


\section{(6.1) Dimension métrique}

Soit $X$ un ensemble muni d'un noyau symétrique $d$.

(6.1.1) (a) Soit $\alpha \in[1,+\infty[$.

On note $\kappa(\alpha)$ le plus petit entier $\kappa$ tel que, pour tout $y \in X$ et tout $r>0$, on puisse trouver au plus $\kappa$ points de $\mathbf{B}_{d}(y, \alpha r)$ de distances mutuelles $>r$ (pour $d$ ).

On note $L(\alpha)$ le plus petit entier $L$ tel que toute boule fermée de rayon $\alpha r$ puisse être recouverte par $L$ boules fermées de rayon $r$. On a toujours $L(\alpha) \leq \kappa(\alpha), L(\beta) \leq L(\alpha)$ et $\kappa(\beta) \leq \kappa(\alpha)$ pour $\beta \leq \alpha$.

(b) On a toujours $L\left(\alpha^{2}\right) \leq L(\alpha)^{2}$.

Donc, s'il existe $\alpha \in] 1,+\infty[$ avec $L(\alpha)$ fini, alors $L(\beta)$ est fini pour tout $\beta \in[1,+\infty[$.

Lorsque $L(\alpha)$ est fini pour un $\alpha \in] 1,+\infty[$, on dit que $(X, d)$ est de dimension métrique finie ou bien que $(X, d)$ possède la "doubling property" (puisque cela équivaut à dire que $L(2)$ est fini).

(6.1.2) (a) La finitude de $\kappa(1)$ n'est pas sans signification, puisque la dimension de De Groot de $(X, d)$ vaut $\kappa(1)-1$ (voir $3.5 \mathrm{~d}$, alors que $L(1)$, qui vaut toujours 1 , est sans intérêt).

(b) En général la finitude de $\kappa(\alpha)$ pour un $\alpha \in] 1,+\infty[$ n'implique pas celle de $\kappa(\beta)$ pour tout $\beta \in[1,+\infty[$. C'est cependant le cas si $d$ vérifie $d(x, y) \leq C[d(x, z)+d(z, y)]$ pour tous $x, y, z \in X$, et en particulier si $d$ est un écart $(C=1)$; en effet on a alors $\kappa(\alpha / 2 C) \leq L(\alpha)$ et la finitude des $\kappa$ équivaut à la finitude des $L$.

(c) Soit $X$ (resp. $Y$ ) un ensemble muni d'un noyau symétrique $d$ (resp. $\delta$ ); une application $g$ de $X$ dans $Y$ est appelée un plongement bi-Lipschitzien de $(X, d)$ dans $(Y, \delta)$ si le noyau $x, y \mapsto \delta(g(x), g(y))$ est bi-Lipschitz équivalent à $d$. Notons qu'un noyau symétrique $d$ sur $X$ vérifie l'inégalité $d(x, y) \leq$ $C[d(x, z)+d(z, y)]$ si et seulement si $\left(X, d^{p}\right)$ peut être plongé de façon biLipschitzienne dans un espace métrique $(Y, \delta)$ pour un $p \in] 0,+\infty[(\operatorname{voir}[1])$.

(d) Tout espace normé de dimension finie est de dimension métrique finie. De plus l'espace métrique $(X, d)$ est de dimension métrique finie si et seulement si $\left(X, d^{p}\right)$ peut être plongé de façon bi-Lipschitzienne dans un $\mathbb{R}^{n}$ muni de la norme euclidienne pour tout $p \in] 0,1[$ (ou pour un $p \in] 0,1$, $\operatorname{voir}[1],[3])$.

\section{(6.2) Espaces $\theta$-géodésiques}

(6.2.1) (a) Soit $\theta \in] 1,2]$; un espace métrique $(X, d)$ est dit $\theta$-géodésique si, pour tous $x, y \in X$, il existe $z \in X$ avec $d(x, z) \leq d(x, y) / \theta$ et $d(z, y) \leq$ $d(x, y) / \theta$. 
Exemples : tout espace géodésique est 2-géodésique ( $z$ est le milieu du segment $[x, y])$; plus généralement, si tout couple $x, y$ de points de $(X, d)$ peut être joint par une courbe située dans $\mathbf{B}_{d}(x, d(x, y) / \theta) \cup \mathbf{B}_{d}(y, d(x, y) / \theta)$, alors $(X, d)$ est $\theta$-géodésique.

(b) Un espace métrique $(X, d)$ est $\theta$-géodésique si et seulement si, pour tous $r>0$ et $x, y \in X, \mathbf{B}_{d}(x, r) \cap \mathbf{B}_{d}(y, r)=\varnothing$ implique $d(x, y)>\theta r$.

Lemme 6.2.2. Soit $\theta \in] 1,2]$ et soit $(X, d)$ un espace métrique $\theta$-géodésique. Soit $\left(y_{i}\right)_{i \in I}$ une famille de points de $X$ de distances mutuelles $>r$. Alors, pour tout $k \geq 1$, on peut colorier les $\left(y_{i}\right)_{i \in I}$ en $\chi(1)^{k}$ couleurs de façon que deux points distincts de même couleur soient toujours de distance $>\theta^{k} r$.

Démonstration. (a) Soit $\left(y_{i}\right)_{i \in I}$ une famille de points de $X$ de distances mutuelles $>r$. Les boules $\mathbf{B}_{d}\left(y_{i}, r\right)$ forment une famille sans liaison qui est colorable en $\chi(1)$ couleurs ; pour cette coloration, deux points $y_{i}$ de même couleur sont de distance $>\theta r$ (voir 6.2.1b).

(b) En itérant la construction faite en (a), on obtient le résultat.

Proposition 6.2.3. Soit $\theta \in] 1,2]$ et soit $(X, d)$ un espace métrique $\theta$ géodésique avec $\chi(1)$ fini. Soit $K$ le plus petit $k$ tel que $\theta^{k} \geq 2$, et soit $L=\chi(1)^{K}$. Alors, pour tout $r>0$, toute boule fermée de rayon $2 r$ peut être recouverte par $L$ boules fermées de rayon $r$.

Démonstration. On se donne $a \in X$ et $r>0$. On choisit une famille maximale $\left(y_{i}\right)_{i \in I}$ de points de distances mutuelles $>r$. On applique 6.2.2 avec $k=K$; on peut donc colorier les $\left(y_{i}\right)_{i \in I}$ en $\chi(1)^{K}$ couleurs de façon que deux points distincts de même couleur soient toujours de distance $>\theta^{K} r \geq$ $2 r$, ce qui implique qu'il n'y a qu'un seul point de chaque couleur et donc que le cardinal de $I$ est $\leq \chi(1)^{K}=L$.

Corollaire 6.2.4. Soit $\theta \in] 1,2]$ et soit $(X, d)$ un espace métrique $\theta$ géodésique. Si $(X, d)$ possède la propriété de recouvrement forte, alors il est nécessairement de dimension métrique finie.

Démonstration. Si $(X, d)$ possède la prF de degré $\leq m$, alors toute famille $\mathcal{B}$ sans liaison de boules fermées est $m$-colorable (car une sous famille de $\mathcal{B}$ recouvrant les centres est nécessairement égale à $\mathcal{B}) ;$ donc $\chi(1) \leq m$ et on applique 6.2.3.

\section{(6.3) Espaces à boules régulières}

(6.3.1) Soit $\beta \geq 1$; un espace métrique $(X, d)$ est dit $\beta$-régulier si, pour tous $x, y \in X$ et tout $t \in] 0,1]$, il existe $z \in X$ avec $d(x, z) \leq(1-(t / \beta)) d(x, y)$ et $d(z, y) \leq \beta t d(x, y)$. Exemple : tout espace géodésique (et en particulier $\mathbb{R}^{n}$ avec la distance associée à une norme) est 1-régulier. 
Lemme 6.3.2. $\quad$ Soit $\beta \geq 1$ et soit $\theta=1+\beta^{-2}$. Tout espace métrique $\beta$-régulier est $\theta$-géodésique.

Démonstration. Soient $x, y \in X, r=d(x, y)$ et $t$ avec $t^{-1}=\beta+\beta^{-1}$. Il existe alors $z \in X$ avec $d(x, z) \leq r-(\operatorname{tr} / \beta)$ et $d(z, y) \leq \beta \operatorname{tr}$. Or on a $1-(t / \beta)=\beta t=1 / \theta$, d'où le résultat.

(6.3.3) Soit $\beta \geq 1$; on dit que la boule $\mathbf{B}_{d}(x, r)$ est $\beta$-régulière si, pour tout $y \in \mathbf{B}_{d}(x, r)$ et tout $\left.\left.t \in\right] 0, r\right]$, il existe $z \in X$ avec $\mathbf{B}_{d}(z, t / \beta) \subset \mathbf{B}_{d}(x, r)$ et $y \in \mathbf{B}_{d}(z, \beta t)$.

Exemple : dans un espace $(X, d) \beta$-régulier (en particulier dans $\mathbb{R}^{n}$ muni de la distance associée à une norme), chaque boule $\mathbf{B}_{d}(x, r)$ est $\beta$-régulière.

En effet soient $y \in \mathbf{B}_{d}(x, r)$ et $\left.t \in\right] 0, r[$; on choisit $z$ par 6.3 .1 si $t<$ $d(x, y)$, et on prend $z=x$ sinon.

\section{(6.4) Parties libres et degrés dans les graphes}

(6.4.1) Soit $S$ un ensemble fini et $A$ une partie de $S^{2}$; on dit que $R \subset S$ est une partie libre du graphe $(S, A)$ si on a $(i, j) \notin A$ pour tous $i, j \in R$ distincts.

Soient $T \subset S$ et $\ell \in T$; on note $d^{-}(\ell, T)$ (resp. $\left.d^{+}(\ell, T)\right)$ le cardinal de $\{i \in T \backslash\{\ell\} \mid(i, \ell) \in A\}$ (resp. $\{i \in T \backslash\{\ell\} \mid(\ell, i) \in A\})$, qu'on appelle degré entrant (resp. degré sortant) de $\ell$ dans $T$. On note $T[\ell]$ l'ensemble des $i \in T$ tels que $(i, \ell) \in A$ ou $(\ell, i) \in A$ ou $i=\ell$.

$T$ est dite de degré entrant $\leq p$ (resp. de degré sortant $\leq p$ ) si tout $\ell \in T$ est de degré entrant (resp. sortant) $\leq p$ dans $T$.

Lemme 6.4.2. Soit $(S, A)$ un graphe; on suppose que toute partie libre est de cardinal $\leq m$.

(a) Alors, quel que soit l'entier $p$, toute partie de $S$ de degré entrant $<p$ est de cardinal $<2 \mathrm{mp}$.

(b) Si de plus $A$ est symétrique (i.e. $(i, j) \in A$ implique $(j, i) \in A$ ), alors toute partie de $S$ de degré $<p$ est de cardinal $\leq m p$.

Démonstration. (a) Supposons que toute partie libre de $S$ soit de cardinal $\leq m$. Soit $T$ une partie finie non vide de $S$ de degré entrant $<p$. On va montrer que $T$ est de cardinal $<2 m p$ en deux étapes :

(a1) soit $U$ une partie finie non vide quelconque de $T$; on a :

$$
\sum_{i \in U} d^{+}(i, U)=\sum_{i \in U} d^{-}(i, U)<p \times \operatorname{cardinal}(U) ;
$$

il existe donc un point $\ell \in U$ de degré sortant $<p$ dans $U$, donc tel que $U[\ell]$ soit de cardinal $<2 p$; 
(a2) on pose $U_{1}=T$; on peut trouver $\ell_{1}$ dans $U_{1}$ tel que $U_{1}\left[\ell_{1}\right]$ soit de cardinal $<2 p$; si $U_{2}=U_{1} \backslash U_{1}\left[\ell_{1}\right]$ est non vide, on peut trouver $\ell_{2}$ dans $U_{2}$ tel que $U_{2}\left[\ell_{2}\right]$ soit de cardinal $<2 p$; et ainsi de suite. Comme $\left\{\ell_{1}, \ldots, \ell_{k}, \ldots\right\}$ est libre, il y a au plus $m$ étapes. On en déduit que $T$ est de cardinal $<2 m p$.

(b) Si de plus $A$ est symétrique, $U[\ell]$ est de cardinal $\leq p$ pour tout $\ell \in U$, et on poursuit la démonstration comme en (a2).

C'est Charles Delorme qui nous a fourni l'énoncé 6.4.2a et la démonstration de la majoration par $2 m p$, alors que, dans une première version, nous avions seulement une majoration en $(m+1)^{p}$; nous tenons à l'en remercier. Quant à 6.4.2b, il est tout à fait classique.

Dans ce qui suit, on fera le même abus de langage qu'au paragraphe 5, consistant à attribuer à $I$ les propriétés vérifiées par la famille $\left(\mathbf{B}_{d}\left(y_{i}, r_{i}\right)\right)_{i \in I}$; de plus on posera parfois $B_{i}=\mathbf{B}_{d}\left(y_{i}, r_{i}\right)$ pour alléger les notations.

Lemme 6.4.3. Soit $X$ un ensemble muni d'un noyau symétrique d et soit $\tau \in\left[1,+\infty\left[\right.\right.$; on suppose que $\kappa(\tau)$ est fini. Soit $\left(\mathbf{B}_{d}\left(y_{i}, r_{i}\right)\right)_{i \in I}$ une famille de boules fermées.

On munit I de la structure de graphe définie par

$$
A=\left\{(i, j) \in I^{2} \mid y_{i} \in B_{j}\right\} .
$$

Alors, toute partie $\tau$-contrôlée de $I$ est de degré entrant $\leq \kappa(\tau)$.

Démonstration. Soit $E$ une partie $\tau$-contrôlée de $I$ et soit $\ell \in E$. Soient $i, j \in E$ distincts avec $y_{i}, y_{j} \in B_{\ell}$; comme $y_{i} \in B_{\ell}$, on a $r_{i} \geq r_{\ell} / \tau$; de même on a $r_{j} \geq r_{\ell} / \tau$; de plus on a $y_{j} \notin B_{i}$ ou $y_{i} \notin B_{j}$, donc $d\left(y_{i}, y_{j}\right)>r_{\ell} / \tau$. Donc le degré entrant de $E$ est $\leq \kappa(\tau)$.

\section{(6.5) Equivalence des propriétés de recouvrement}

Lemme 6.5.1. Soit $\left(\mathbf{B}_{d}\left(y_{i}, r_{i}\right)\right)_{i \in I}$ une famille de boules fermées d'un espace métrique de dimension métrique finie et dont les boules sont $\beta$-régulières. Alors, dans toute partie de I d'ordre $\leq p$, toute $\tau$-configuration est de cardinal $\leq p \times \kappa(\beta(\beta+\tau))$.

Démonstration. Soit $E \subset I$ une $\tau$-configuration de centre $\ell$ d'ordre $\leq p$. Soit $Q_{\ell}$ une famille maximale de points de $\mathbf{B}_{d}\left(y_{\ell},(\beta+\tau) r_{\ell} / \tau\right)$ de distances mutuelles $>r_{\ell} / \tau \beta$. La famille $Q_{\ell}$ est donc de cardinal $\leq \kappa(\beta(\beta+\tau))$.

Fixons $i \in E \backslash\{\ell\}$. Soit $u$ un élément de $B_{\ell} \cap B_{i}$; comme on a $r_{\ell} / \tau \leq r_{i}$, il existe une boule $C_{\ell}=\mathbf{B}_{d}\left(z, r_{\ell} / \tau \beta\right)$ incluse dans $B_{i}$ et telle que $\mathbf{B}_{d}\left(z, \beta r_{\ell} / \tau\right)$ contienne $u$; on a donc $d\left(z, y_{\ell}\right) \leq d(z, u)+d\left(u, y_{\ell}\right) \leq(\beta+\tau) r_{\ell} / \tau$. La boule $C_{\ell}$ contient un point de $Q_{\ell}$, il en est donc de même de la boule $B_{i}$. Par ailleurs $B_{\ell}$ contient évidemment aussi un point de $Q_{\ell}$.

On trouve : $\operatorname{cardinal}(E) \leq \operatorname{ordre}(E) \times \operatorname{cardinal}\left(Q_{\ell}\right)<p \kappa(\beta(\beta+\tau))$. 
Si on avait seulement une inégalité du type $d(x, y) \leq C[d(x, z)+d(z, y)]$ on obtiendrait le même résultat avec $\kappa(\beta(\beta+\tau))$ remplacé par $\kappa(C \beta(\beta+\tau))$

Proposition 6.5.2. Soit $(X, d)$ un espace métrique de dimension métrique finie dont les boules sont $\beta$-régulières. On a alors :

(a) $\mathbf{c}(\tau) \leq \mathbf{o}(\tau) \times \kappa(\beta(\beta+\tau))$ pour tout $\tau \geq 1$, et en particulier $\mathbf{c}(1) \leq \mathbf{n}_{D} \times \kappa(\beta(\beta+1))$;

(b) $\mathbf{o}(\tau)<2 \mathbf{n}_{D} \times[\kappa(\tau)+1]$ pour tout $\tau>1$.

On en déduit que :

(c) $\mathbf{N}_{B}<2 \mathbf{n}_{D} \times[\kappa(\tau)+1] \times \kappa(\beta(\beta+\tau))$ pour tout $\tau>1$;

(d) $\mathbf{N}_{D}<\mathbf{n}_{D} \times \kappa(\beta(\beta+1))$.

\section{Démonstration.}

(a) On prend une partie $E \tau$-contrôlée d'ordre $\leq p$; dans $E$ (par 6.5.1), toute $\tau$-configuration est de cardinal $\leq p \times \kappa(\beta(\beta+\tau))$. Cela donne le résultat (en remarquant que $\mathbf{o}(1)=\mathbf{n}_{D}$ ).

(b) Soit $\left(\mathbf{B}_{d}\left(y_{i}, r_{i}\right)\right)_{i \in I}$ une famille de boules $\tau$-contrôlée de $(X, d)$. Soit $a \in X$ et soit $E=\left\{i \in I \mid a \in B_{i}\right\}$; toute partie sans liaison de $E$ est de cardinal $\leq \mathbf{n}_{D}$. On munit $E$ de la structure de graphe définie par $A=\left\{(i, j) \in E^{2} \mid y_{i} \in B_{j}\right\} ;$ donc (par 6.4.3) $E$ est de degré entrant $<\kappa(\tau)+1$; donc (par 6.4.2) $E$ est de cardinal $<2 \mathbf{n}_{D} \times[\kappa(\tau)+1]$.

(c) est la conséquence de (a) et (b) :

en effet on a

$\mathbf{N}_{B} \leq \mathbf{c}(\tau) \leq \mathbf{o}(\tau) \times \kappa(\beta(\beta+\tau))<2 \mathbf{n}_{D} \times[\kappa(\tau)+1] \times \kappa(\beta(\beta+\tau))$.

(d) est la conséquence de (a) et de $\mathbf{o}(1)=\mathbf{n}_{D}$ :

en effet on a

$$
\mathbf{N}_{D} \leq \mathbf{c}(1) \leq \mathbf{o}(1) \times \kappa(\beta(\beta+1))=\mathbf{n}_{D} \times \kappa(\beta(\beta+1)) .
$$

\section{Corollaire 6.5.3.}

(a) Soit $(X, d)$ un espace métrique de dimension métrique finie dont les boules sont $\beta$-régulières. Alors la propriété de recouvrement faible équivaut à la propriété de recouvrement forte.

(b) Soit $(X, d)$ un espace métrique $\beta$-régulier possédant la propriété de recouvrement faible; alors l'espace $(X, d)$ possède la propriété de recouvrement forte si et seulement si il est de dimension métrique finie. 
Démonstration. (a) Si $(X, d)$ vérifie la propriété de recouvrement faible, alors $\mathbf{n}_{D}$ est fini ; par 6.5.2c, $\mathbf{N}_{B}$ est fini, donc $(X, d)$ vérifie la propriété de recouvrement forte.

(b) Comme $(X, d)$ est $\beta$-régulier, ses boules sont $\beta$-régulières (voir 6.3.3) et il est $\theta$-géodésique pour $\theta=1+\beta^{-2}$ (voir 6.3 .2 ) ; l'équivalence résulte dans un sens de (a), et dans l'autre de 6.2.4.

(6.5.4) Si $(X, d)$ est un espace métrique géodésique, on peut, sans supposer que la dimension métrique soit finie, majorer $\mathbf{c}(1)$ à l'aide de $\mathbf{N}_{B}$. De fait on a alors $\mathbf{c}(1) \leq \mathbf{N}_{B} \times \mathbf{n}_{D} \leq\left(\mathbf{N}_{B}\right)^{2}$.

En effet posons $k=\mathbf{N}_{B}$; soit $E$ une 1-configuration de centre $\ell$, supposée sans liaison ; on peut partager $E$ en $k$ parties 1-colorables, $E\left(c_{1}\right), \ldots, E\left(c_{k}\right)$;

- si $E(c)$ contient $\ell$, on a $E(c)=\{\ell\}$;

- sinon, pour tous $i, j \in E(c)$ distincts, on a :

$$
\text { et } \quad \begin{aligned}
d\left(y_{i}, y_{j}\right) & >r_{i}+r_{j} \quad(\operatorname{car}(X, d) \text { est géodésique }) \\
\text { et } & d\left(y_{\ell}, y_{i}\right) \leq r_{i}+r_{\ell} \leq r_{i}+r_{j}
\end{aligned}
$$

donc la famille des boules $\left(\mathbf{B}_{d}\left(y_{i}, d\left(y_{\ell}, y_{i}\right)\right)\right)_{i \in I}$ est sans liaison, donc $E(c)$ est de cardinal $\leq \mathbf{o}(1)=\mathbf{n}_{D}$.

\section{Tableau des principales notions et notations}

\section{Principales notions :}

bi-Lipschitz équivalent 1.3

cardinal "grand" ou "petit" au sens d'Ulam 2.6

cardinal mesurable, cardinal $U$-mesurable 2.6

$\tau$-configuration de centre $\ell \quad 5.1$

degré entrant ou sortant 6.4

dimension de De Groot 3.5

dimension de Nagata 4.7

dimension métrique $\quad 6.1 .1$

"doubling property" 6.1 .1

écart 1.3

espace géodésique 1.4

espace à courbure $\geq 0 \quad 1.4$

espace séparable 2.6

espace $\alpha$-séparable 2.6

espace fortement $\mathcal{A}$-mesurable $\quad 2.7 .1$

espace $\theta$-géodésique $\quad 6.2$

espace à boules $\beta$-régulières $\quad 6.3$

espace $\beta$-régulier $\quad 6.3$

famille de boules d'ordre $\leq m \quad 1.1$

famille de boules colorable en $m$ couleurs 1.1

famille de boules à rayons bornés 1.1 
famille de boules à rayons discrets 1.1

famille de boules sans liaison 4.1 et 5.1

famille de boules $\tau$-contrôlée $\quad 5.1$

inégalité maximale 4.3

jauge 2.4

$L$-mesure 2.6

mesure $\alpha$-additive $\quad 2.6$

mesure diffuse 2.6

noyau symétrique 1.3

partie positivement chargée par $\nu \quad 2.5$

partie de dimension de Nagata $\leq n$ dans $(X, d) \quad 4.8$

partie libre dans un graphe 6.4

partition pointée $\gamma$-fine 2.4

plongement bi-Lipschitzien $\quad 6.1 .2$

propriété de recouvrement faible de degré $\leq m \quad 1.2$

(idem) restreinte aux familles de boules à rayon constant 1.2

(idem) restreinte aux boules centrées dans $Y \quad 1.2$

(idem) restreinte aux familles de boules de rayon $r \quad 3$

(idem) restreinte aux boules centrées dans $Y$ et de rayon $<s \quad 4.8$

propriété de recouvrement forte de degré $\leq m \quad 1.2$

propriété de De Giorgi de degré $\leq m \quad 1.2$

quotient de dérivation 2.1

segment géodésique 1.4

support de $\lambda$ relatif à $d \quad 2.6 .1$

théorème de Vitali-Carathéodory 2.4

théorème de Nagata-Ostrand 4.8

théorème de Preiss 4.8

tribu de Baire 1.3 et 2.4

ultramétrique 3.5

\section{Principales notations :}

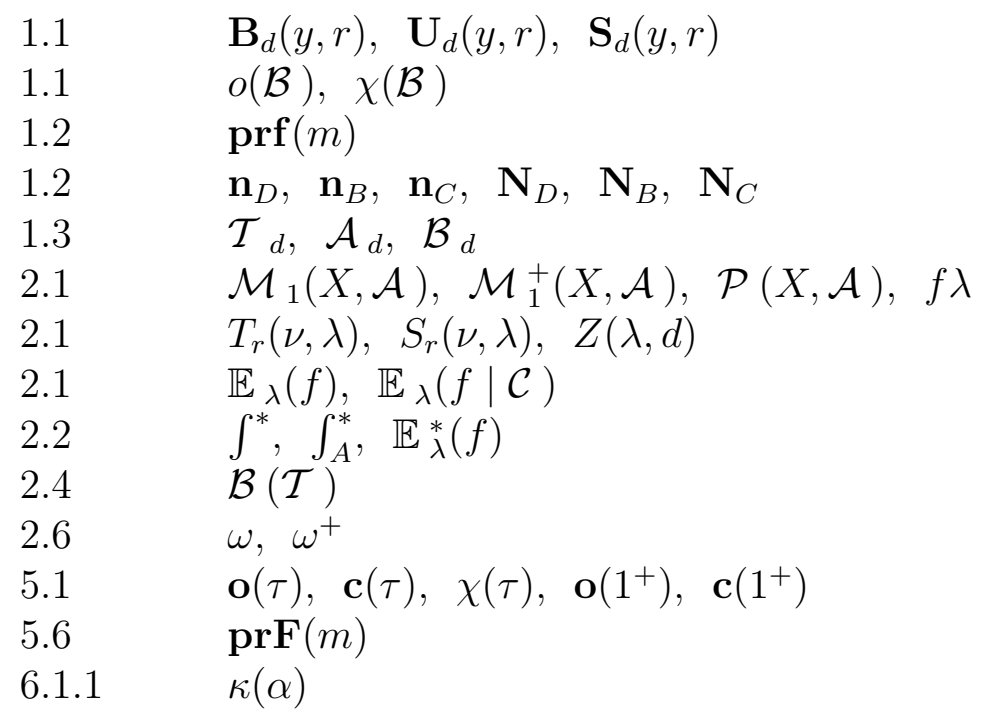




\section{References}

[1] Assound, P.: Espaces métriques, plongements, facteurs. Thèse de doctorat, Publications Mathématiques d'Orsay, No. 223-7769. U.E.R. de Mathématique, Université de Paris XI, Orsay, 1977.

[2] Assound, P.: Sur la distance de Nagata. C. R. Acad. Sci. Paris Sér. I Math. 294 (1982), 31-34

[3] Assouad, P.: Plongements lipschitziens dans $\mathbb{R}^{n}$. Bull. Soc. Math. France 111 (1983), 429-448

[4] Assouad, P. and Quentin de Gromard, T.: Dérivation des mesures à l'aide de pseudo-boules. C. R. Acad. Sci. Paris Sér. I Math. 320 (1995), $1065-1068$

[5] Assouad, P. And Quentin de Gromard, T.: Dimension topologique et propriétés de recouvrement (travail en cours).

[6] Besicovitch, A.S.: A general form of the covering principle and relative differentiation of additive functions. Proc. Cambridge Philos. Soc. 41 (1945), 103-110

[7] Berger, M.: La géométrie métrique des variétés riemanniennes (variations sur la formule $a^{2}=b^{2}+c^{2}-2 b c \cos \alpha$ ). In The mathematical heritage of Élie Cartan (Lyon, 1984), 9-66. Astérisque (1985), numero hors série.

[8] Billingsley, P.: Convergence of Probability Measures. John Wiley, New York-London-Sydney, 1968.

[9] Burago, D., Burago, Y. and Ivanov, S.: A course in metric geometry. Graduate Studies in Mathematics 33. American Mathematical Society, Providence, RI, 2001.

[10] Cheeger, J. and Ebin, D.G.: Comparison theorems in Riemannian geometry. North-Holland Mathematical Library 9. North-Holland Publishing Co., 1975.

[11] Chang, C.C. And Keisler, H.J.: Model Theory, second edition. Studies in Logic and the Foundations of Mathematics 73. North-Holland, Amsterdam-New York-Oxford, 1977.

[12] Cygan, J.: Subadditivity of homogeneous norms on certain nilpotent Lie groups. Proc. Amer. Math. Soc. 83 (1981), 69-70.

[13] Davies, R.O. And Schuss, Z.: A proof that Henstock's integral includes Lebesgue's. J. London Math. Soc. (2) 2 (1970), 561-562

[14] Dellacherie, C. And Meyer, P.A.: Probabilités et potentiel. Chapitres I à $I V$. Publications de l'Institut de Mathématique de l'Université de Strasbourg, No. XV. Actualités Scientifiques et Industrielles 1372. Hermann, Paris, 1975.

[15] Dellacherie, C. And Meyer, P. A.: Probabilités et potentiel. Chapitres $V$ à VIII. Théorie des martingales. Actualités Scientifiques et Industrielles 1385. Hermann, Paris, 1980. 
[16] De Giorgi, E., Colombini, F. And Piccinini, L. C.: Frontiere orientate di misura minima e questioni collegate. Scuola Normale Superiore, Pisa, 1972.

[17] De Groot, J.: On a metric that characterizes dimension. Canad. J. Math. 9 (1957), 511-514

[18] De Guzmán, M.: Differentiation of integrals in $\mathbb{R}^{n}$. Lecture Notes in Mathematics 481. Springer-Verlag, Berlin-New York, 1975.

[19] De Guzmán, M.: Real variable methods in Fourier analysis. NorthHolland Mathematics Studies 46. North-Holland, Amsterdam-New York, 1981.

[20] Federer, H.: Geometric Measure Theory. Die Grundlehren der mathematischen Wissenschaften 153. Springer-Verlag, New York, 1969.

[21] Füredi, Z. And Loeb, P.A.: On the best constant for the Besicovitch covering theorem. Proc. Amer. Math. Soc. 121 (1994), 1063-1073.

[22] Jech, Th.: Set Theory. Academic Press, 1978.

[23] Kellerer, H. G.: Baire sets in product spaces. In Measure theory (Oberwolfach 1979), 38-44. Lecture Notes in Math. 794. Springer, Berlin, 1980.

[24] Korányi, A. and Reimann, H. M.: Foundations for the theory of quasiconformal mappings on the Heisenberg group. Adv. Math. 111 (1995), 1-87.

[25] Lang, U. and Schlichenmaier, T.: Nagata dimension, quasisymmetric embeddings, and Lipschitz extensions. Int. Math. Res. Not. 2005 58, 36253655 .

[26] Mattila, P.: Differentiation of measures on uniform spaces. In Measure theory (Oberwolfach 1979), 261-283. Lecture Notes in Math. 794. Springer, Berlin, 1980.

[27] Meyer, P.A.: Probabilités et potentiel. Publications de l'Institut de Mathématique de l'Université de Strasbourg, No. XIV. Actualités Scientifiques et Industrielles 1318. Hermann, Paris, 1966.

[28] Morse, A. P.: Perfect blankets. Trans. Amer. Math. Soc. 61 (1947), 418-442.

[29] McShane, E. J.: Unified integration. Pure and Applied Mathematics 107. Academic Press, New York, 1983.

[30] Nagata, J. I.: On a special metric and dimension. Fund. Math. 55 (1964), 181-194.

[31] Ostrand, P. A.: A conjecture of J. Nagata on dimension and metrization. Bull. Amer. Math. Soc. 71 (1965), 623-625.

[32] Охтовy, J. C.: Measure and Category. A survey of the analogies between topological and measure spaces. Graduate Texts in Mathematics 2. Springer-Verlag, New York-Berlin, 1971.

[33] Preiss, D.: Dimension of metrics and differentiation of measures. In General topology and its relations to modern analysis and algebra, $V$ (Prague, 1981), 565-568. Sigma Ser. Pure Math. 3. Heldermann, Berlin, 1983. 
[34] PU, H.W.: Another proof that Riemann-complete integral includes the Lebesgue integral. Bull. Soc. Roy. Sci. Liège 41 (1972), 250-251.

[35] Rinow, W.: Die innere Geometrie der metrischen Raüme. Die Grundlehren der mathematischen Wissenschaften 105. Springer-Verlag, Berlin-Göttingen-Heidelberg, 1961.

[36] Stein. E. M.: Singular integrals and differentiability properties of functions. Princeton Mathematical Series 30. Princeton University Press, Princeton, N.J. 1970

[37] Sullivan, J. M.: Sphere packings give an explicit bound for the Besicovitch covering theorem. J. Geom. Anal. 4 (1994), 219-231.

[38] Sawyer, E. And Wheeden, R. L.: Weighted inequalities for fractional integrals on Euclidean and homogeneous spaces. Amer. J. Math. 114 (1992), 813-874.

[39] Ulam, S.: Zur Masstheorie in der allgemeinen Mengenlehre. Fund. Math. 16 (1930), 140-150.

[40] Van der VaArt, A. W. And Wellner, J. A.: Weak convergence and empirical processes. With applications to statistics. Springer Series in Statistics. Springer-Verlag, New York, 1996.

[41] Zanzotto, P. A.: On measure derivation in metric spaces. Mathematika 36 (1989), 349-358.

[42] Ziemer, W.P.: Weakly differentiable functions. Sobolev spaces and functions of bounded variation. Graduate Texts in Mathematics 120. SpringerVerlag, New York, 1989.

Recibido: 13 de septiembre de 2004

Revisado: 9 de febrero de 2005

Patrice Assouad CNRS et U. Paris XI

Centre d'Orsay, bât. 425

91405 Orsay, France

Patrice.Assouad@math.u-psud.fr

Thierry Quentin de Gromard

U. Paris XI

Centre d'Orsay, bât. 425

91405 Orsay, France

Thierry.Quentin@math.u-psud.fr 